\title{
Supramolecular organization in confined nanospaces
}

Gloria Tabacchi*[a]

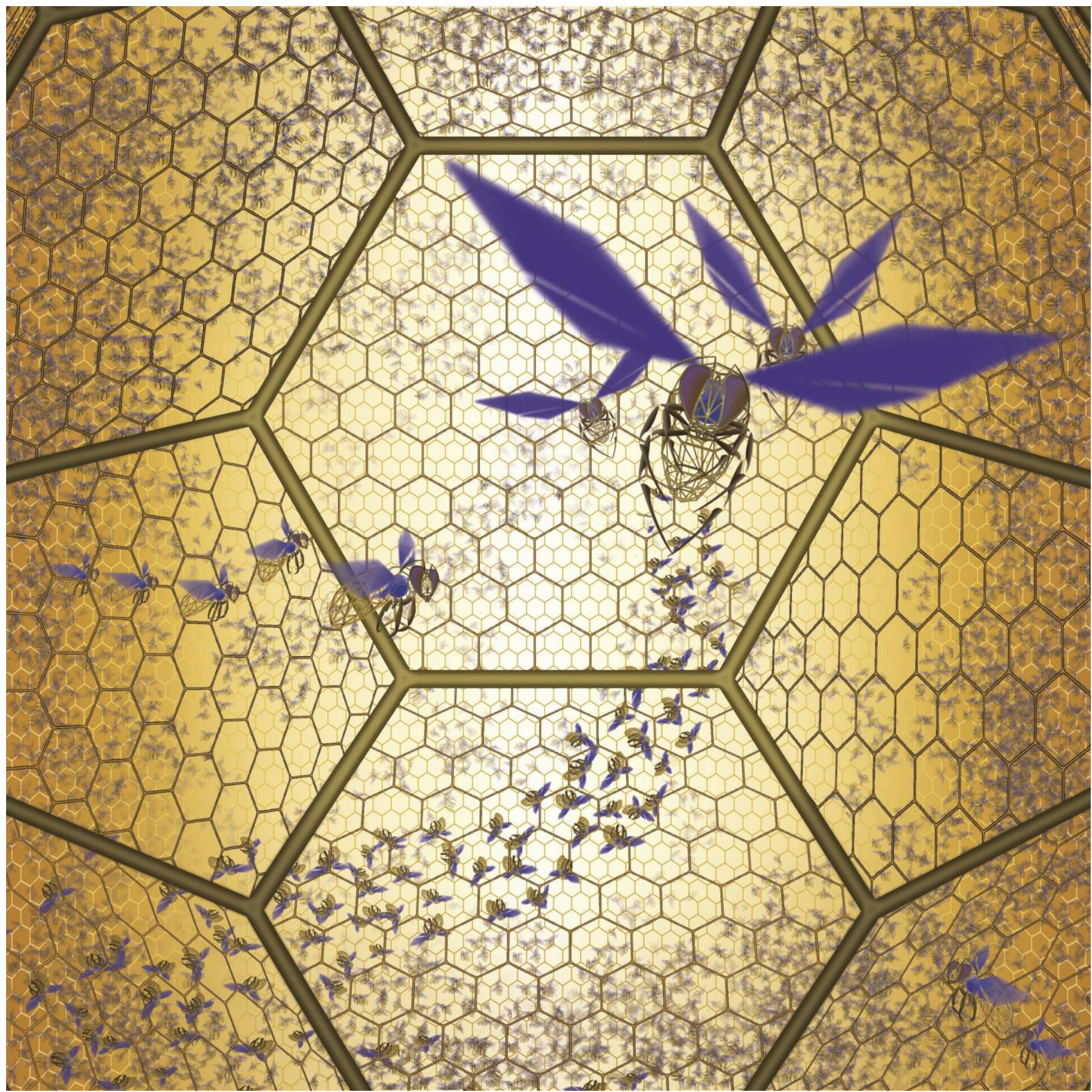


Abstract: Empty spaces are abhorred by nature that immediately rushes in to fill the void. Humans have learnt pretty well how to make ordered empty nanocontainers, and to get useful products out of them. When such an order is imparted to molecules, new properties may appear, often yielding advanced applications. This review illustrates how the organized void space inherently present in various materials - zeolites, clathrates, mesoporous silica/organosilica, and metal organic frameworks (MOF), for example, can be exploited to create confined, organized, and self-assembled supramolecular structures of low-dimensionality. Features of the confining matrices relevant to organization are presented with special focus on molecular-level aspects. Selected examples of confined supramolecular assemblies- from small molecules to quantum dots or luminescent species- are aimed to show the complexity and potential of this approach. Natural confinement (minerals) and hyperconfinement (high pressure) provide further opportunities to understand and master the atomistic-level-interactions governing supramolecular organization under nanospace restrictions.

\section{Introduction}

Supramolecular organization is a central idea in chemistry and a driving force in technology. ${ }^{[1-3]}$ It refers to the formation of correlated domains of molecules or nanospecies exhibiting collective properties or new functionalities. ${ }^{[4-8]}$ Molecular machines ${ }^{[6,9-11]}$ and photonic antennas ${ }^{[12]}$ are classic examples of supramolecular structures. The assembly process may occur through ferroelectricity or ferromagnetism, by molecular recognition between specifically designed species, or with the help of an external agent: for example light, electric/magnetic fields or space confinement. ${ }^{[13]}$ In the latter case, matrices with regular porous networks are particularly effective, because their voids can be exploited as nanosized receptors for matter to create confined, supramolecular structures of lowdimensionality. ${ }^{[14]}$ Empty space architectures are a distinctive feature of various materials: zeolites, crystalline mesoporous silica, or metal organic frameworks, among others. Because of the regular distribution of nanosized empty space, such frameworks may be ideally considered as the negative image of highly structured nanomaterials, constituted by arrays of molecules, quantum dots, or molecular wires. ${ }^{[15]}$ When confined in those matrices, guest species not only are subjected to the geometrical constraints of the cavities, but are also prone to be remotely and individually controlled. ${ }^{[16,17]}$

Nanoscale confinement has long been used as a way to enhance or modify chemical reactivity, as documented in several reviews on catalysis, ${ }^{[18-22]}$ photochemistry, ${ }^{[23-26]}$ and charge-transfer processes. ${ }^{[27,28]}$ Equally important are situations where no covalent bonds are broken or formed, and the porous matrix is used essentially as an organizing medium. In such host-guest or

\section{[a] G. Tabacchi}

Department of Science and High Technology

University of Insubria

Via Valleggio, 9 I-22100 Como (Italy)

E-mail:gloria.tabacchi@uninsubria.it

inclusion composites, the guest species are bound inside the cavities or channels of the host by non-covalent forces such as hydrogen bonding, halogen bonding, electrostatics and van der
Waals interactions. This work aims to give an overview of hostguest materials whose potential applications hold concrete promises in materials science. Composites of this kind for electronics, photonics or sensing, were first obtained by incorporating molecules and nanoparticles inside zeolites. ${ }^{[14,17,29]}$ These represent a kind of archetype for other systems and are the main subject of this story; nonetheless, discussion is extended to other hosts that have widened the application range while presenting new challenges (Figure 1). ${ }^{[30,31]}$ Focus is driven on the molecular-level features of the confined assemblies, whose knowledge is pivotal for progress in applications. In this sense, the relevance of natural porous matrices to probe fundamental aspects of host-guest interactions and confinement is acknowledged. Among viable strategies for the realization of composites of potential technological interest, particular attention is here devoted to the use of high pressures.

The concepts of organization and order are not equivalent and need to be defined. Order is usually associated to the symmetry and periodicity of crystals (long-range order): for example, the deviation from strict periodicity in crystallography is often called disorder. In general, a confining crystalline matrix does not impart its symmetry and periodicity to the incorporated species. This happens because host-guest materials are non-stoichiometric compounds - hence, "it is not necessary for guest molecules to occupy every available gap in the host structure before a stable complex is formed" ${ }^{[32]}$ Very early this fact was recognized by Barrer, that classified host-guest complexes according to the distribution of guest species within the framework and observed that these compounds manifested appreciable departures from stoichiometry. ${ }^{[32]}$ Practically, all materials herein presented fall in this category: because they are not "strictly periodic", they are inherently dominated more by disorder rather than by order. Yet the arrangement of confined species is far from being random: confinement imposes spatial correlations to the guests, and the supramolecular patterns thus formed, depending on the observed length scales and adopted experimental conditions, often exhibit a certain regularity, designed as correlated disorder. ${ }^{[33]}$

Organization stems from the space restrictions imposed by the confining matrix to the included species and often implies a change in their physico-chemical properties. For example, metal clusters confined in a regular, three-dimensional system of pores are organized in a three dimensional lattice, while chromophores incorporated inside non-intersecting nanochannels are organized into one-dimensional arrays. Such organized assemblies have electronic absorption, luminescence, and energy transfer properties different from those of a fluid solution or dispersion of the same species. The arrangement of dye molecules in linear arrays, for example, allows for a very efficient and unidirectional FRET (Förster Resonance Energy Transfer) ${ }^{[34]}$ process - a distance-dependent, nonradiative energy transfer mechanism exploited in solar energy harvesting antenna systems (Figure $1 b, d, e){ }^{[35,36]}$ Hence, nanoscale confinement is by itself an organizing agent, thus the arrangement of guests in the porous matrix will be defined as their organization - as common practice in the related literature (see e.g. Ref. ${ }^{[14,16,36-38]}$ )

Organization does not imply crystallographic order: besides nonstoichiometry (non-uniform occupancy of the host pores), there are other sources of disorder for the guest species. At the molecular scale, the arrangement of the guests is determined by a complex mix of intermolecular and host-guest interactions, depending on temperature, pressure and concentration of the guests, among other factors. The confined species generally preserve a certain freedom of movement, like rotational or wobbling motions inside cavities, and longitudinal displacement 
along nanochannels. This atomic-scale dynamics is responsible of the "dynamic disorder" in crystallography: the refined structure typically features a multiplicity of positions with fractional occupancy (as shown e.g. in Figure 1a), and provides only an average picture of the guests. Also, inclusion composites often show "static" disorder - which is related to the symmetry of the guests, their repartition inside the host, and inhomogeneity of the host. These situations are rather common and should be ideally tackled by multitechnique characterization strategies.

Most of the herein discussed composites are functional in spite of disorder: for example, the electronic transition dipole moments of the dyes incorporated in zeolite $L$ (Figure $1 b$ ) are approximately aligned, ensuring thus an extremely high speed for exciton transport. ${ }^{[39,40]}$ Technically, this is good enough for use in solar energy concentrators, sensors or solar cells. ${ }^{[28]}$ Of course, there is still room for improvement, which requires a thorough, atomisticlevel characterization of the material. Indeed, in spite of great progresses in experimental techniques (sketched in Chapter 2), important details still remain very difficult to capture, especially when fine movements of molecules or groups of atoms are the main characters of the play. The key role of computational approaches in revealing these aspects is highlighted throughout this paper, which is structured as follows.

Chapter 3: confining matrices are instrumental in the organization of the included species. This part provides an overview of common porous scaffolds - zeolites, clathrates, silica mesopores, and framework materials.

Chapter 4: confined nanostructures (metal clusters, quantum dots, nanowires, etc.) played a key role in the development of nanoporous-based materials for advanced applications ${ }^{[14,41,42]}$ and are now experiencing impressive progress. ${ }^{[43]}$

Chapter 5: confined water and water-ion clusters is a topic of both historic and fundamental value (see ref. ${ }^{[44,45]}$ for a review) here, the focus is on water-cation assemblies in natural zeolites.

Chapter 6: confined halogen molecules in nanochannels form relatively simple composites, yet endowed with interesting properties, such as semiconductor behavior. ${ }^{[46]}$

Chapter 7: organic chromophores encapsulated in nanosized containers show exceptional optical properties (Figure 1). Confinement greatly enhances their emission intensity by contrasting the formation of undesired aggregates. ${ }^{[47,48]}$ These materials find application in solar energy harvesting, ${ }^{[49]}$ photonics, ${ }^{[50,51]}$ and nano-diagnostics technology. ${ }^{[52]}$ Here, the focus is on the arrangement of dye molecules and the dynamic guest-guest/host-guest interactions governing the optical anisotropy of the materials.

Chapter 8: hyperconfinement, i.e. the use of high pressure to drive molecular species inside nanoporous materials. ${ }^{[53]}$ In this case, the self-assembly process is controlled by both the confining matrix and the imposed pressure. Recently observed phenomena such as pressure-induced polymerization ${ }^{[54]}$ or formation of complex supramolecular patterns ${ }^{[55]}$ candidate hyperconfinement as an intriguing route to new materials.

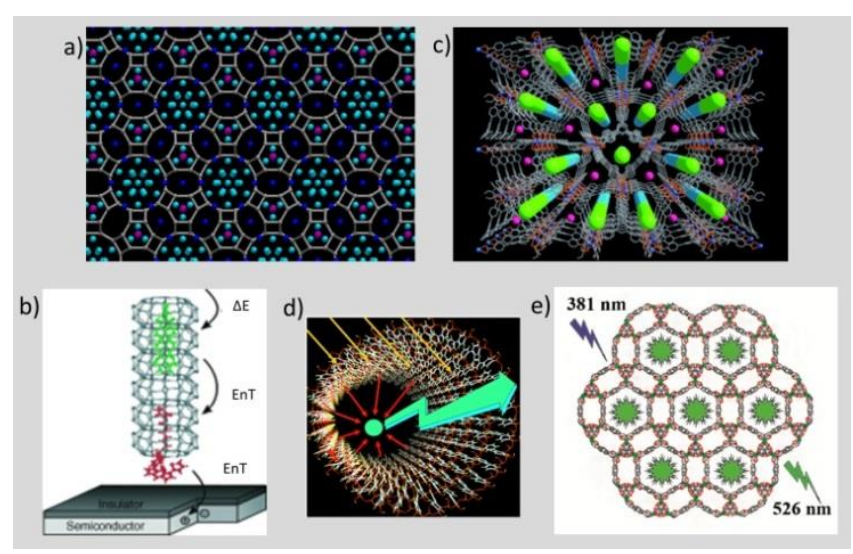

Figure 1. Confining matrices and light-harvesting applications. a) X-ray structure of perlialite ${ }^{[56-58]}\left(\mathrm{K}_{8} \mathrm{Tl}_{4}\left[\mathrm{Al}_{12} \mathrm{Si}_{24} \mathrm{O}_{72}\right] \cdot 20 \mathrm{H}_{2} \mathrm{O}\right)$ - a mineral isostructural to zeolite $\mathrm{L}^{[59]}$ (LTL framework type $)^{[58,60]}$. Cationic and water sites have fractional occupancy. Color code: grey, framework (Si or $\mathrm{Al}$ ); dark blue, $\mathrm{K}^{+}$sites; purple, $\mathrm{Tl}^{+}$sites; light-blue, water sites. [lllustration created with VMD, using X-ray positions from Refs ${ }^{[57,58]}$ b) Zeolite $L$ antenna system. ${ }^{[61]}$ A luminescent donor dye (green) inside zeolite $L$ (grey) functionalized with acceptor dye (in red) at the crystal termination and interfaced with a semiconductor. Upon light irradiation, the composite undergoes FRET ${ }^{[34,39]}$ and energy is transferred to the semiconductor. [Reproduced by permission from Wiley- $\mathrm{VCH}] .^{[61]} \mathrm{c}$ ) Pyridinium hemicyanine dye (green balls) incorporated into a MOF give a material with non-linear optical behavior. ${ }^{[62]}$ [Reprinted from ref. ${ }^{[62]}$ with permission from Wiley-VCH]. d) A periodic mesoporous organosilica bears chromophores within its pore walls and adsorbs light. Energy is transferred to the acceptor dye in the channel (green dot), yielding 100\% emission enhancement. [Reproduced by permission from Wiley-VCH]. ${ }^{[63]}$ e) This zeolitic metal-organic-framework (ZMOF) with included dyes (in green), has excellent light-harvesting properties. [Adapted from Ref. ${ }^{[64]}$ with permission from The Royal Society of Chemistry]

\subsection{Short history}

The use of porous matrices to create new composites is not a new idea. The first of those materials was realized by the Maya (Figure 2). ${ }^{[65]}$ By incorporating the indigo dye in the pores of a clay mineral palygorskite, ${ }^{[66]}$ these ancient craftsmen obtained a pigment of exceptional resistance to harsh conditions, whose color could be finely tuned via a suitable choice of the process conditions. ${ }^{[66]}$ Only much later it was recognized that the stability of the pigment was due to the inclusion of the dye inside the cavities of the clay:[67-69] In particular, the main stabilizing hostguest interaction is the coordination of the carbonyl group of indigo to the $\mathrm{Al}^{3+}$ ions at the edge of the palygorskite cavities (Figure 2), while similar $\mathrm{Mg}^{2+}$-dye interactions can also be present. ${ }^{[67]}$ Computational analyses also suggested that the thermal treatment employed in the pigment preparation eliminates some structural water molecules coordinated to the $\mathrm{Al}^{3+}$ ions in the clay, thus allowing for the binding of indigo. ${ }^{[70]}$ Maya-blue mimics based on clay minerals are now of relevance for the environmentally-friendly production of colorants, and a fertile soil for advanced materials and sustainable applications alike. ${ }^{\text {[1-74] }}$ For example, cointercalation of water-soluble indigo/thioindigo derivatives in layered double hydroxides leads to fluorescence quenching, suggesting efficient energy transfer for the hybrid material. ${ }^{[74]}$ Also, intercalation of a metal complex within clay nanoplatelets enables to control polarization of the emitted light. ${ }^{[72]}$ Similar strategies can be applied also to hydrophobic dyes, producing hybrid materials with well-defined orientation of the included molecules, thus anisotropic optical properties. ${ }^{[73]}$ 


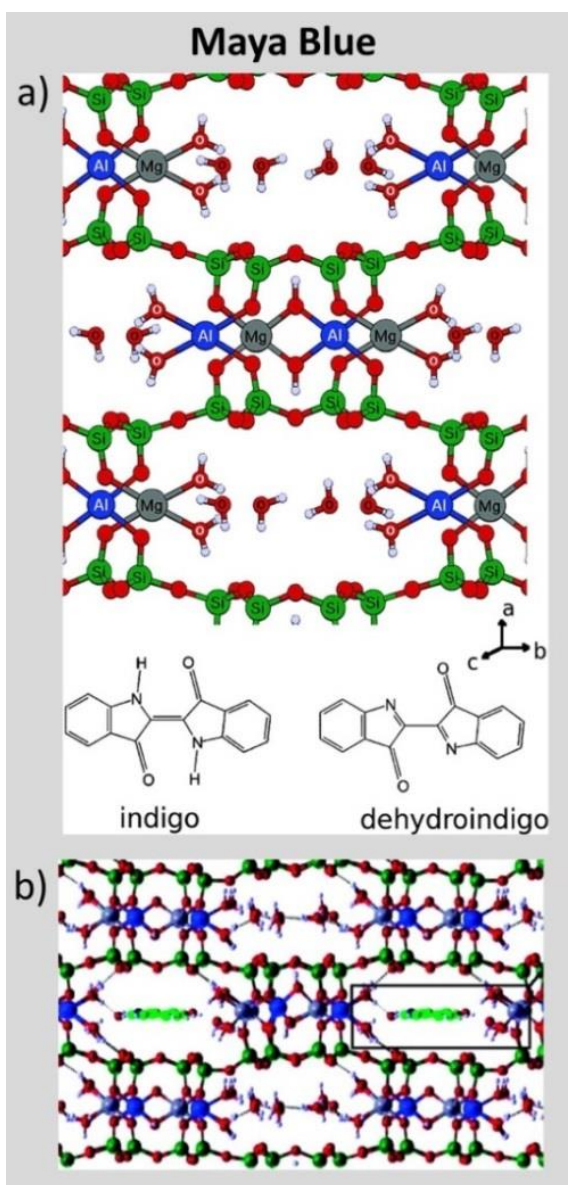

Figure 2. The Maya Blue pigment. a) The inorganic and organic components of Maya blue. The palygorskite clay: $\mathrm{O}$ atoms of structural water bound to $\mathrm{Al}$ are labeled in white, $\mathrm{O}$ atoms of zeolitic water are labeled in black. The indigo and dehydroindigo dyes. b) The Maya Blue pigment, a colorant extraordinarily resistant to humidity, heat, and chemical/photochemical attack, is formed by the intercalation of indigo in the palygorskite channels upon loss of water. [Adapted with permission from Ref. ${ }^{[67]}$ Copyright 2009 American Chemical Society]

Among the first supramolecular compounds, clathrate hydrates ${ }^{[75]}$ occupy a leading position: they were first reported by Davy, Faraday and probably Priestley, over 200 years ago, and are likely the host-guest materials best characterized. They are crystalline solids formed by guest molecules trapped in cages of hydrogen bonded water molecules. ${ }^{[76]}$ Gas hydrates are also found naturally (offshore, under permafrost and in glaciers), ${ }^{[7]}$ and these ones are minerals, according to the American Geological Association. Due to the long history of the field, and to the high relevance of hydrates for industrial and environmental issues, ${ }^{[78]}$ an impressive amount of structural and thermodynamic data has been gathered, ${ }^{[75]}$ which also makes them a choice model for the study of host-guest interactions from a fundamental viewpoint. The field developed around the 1950's, when single crystal structures of hydrates became available. ${ }^{[79]}$ Barrer made prominent contributions to this area, ${ }^{[32]}$ as well as to the first zeolite syntheses ${ }^{[80]}$ and metal incorporation experiments. ${ }^{[81]}$

The idea of thoughtfully exploiting porous materials as ordering matrices for advanced optoelectronic functionalities was the thesis of a seminal work envisioning the technological potential of zeolites in electro-optical devices. ${ }^{[14]}$ Such roadmap was followed by other reports on zeolite use in sensing devices, and ionic conductivity, ${ }^{[16,17,82,83]}$ and was paralleled by progress in zeolite syntheses. ${ }^{[84,85]}$ Porous aluminophosphates, ${ }^{[86,87]}$ oxides, ${ }^{[88]}$ and chalcogenides ${ }^{\left[{ }^{[9-93]}\right.}$ were realized and tested as nanocontainers of photoactive molecules, clusters, or quantum dots. The resulting materials exhibited non-linear optical behavior, such as second harmonic generation, ${ }^{[94]}$ and were used in devices like zeolitebased lasers ${ }^{[95]}$ or sensors. ${ }^{[90]}$

Periodic mesoporous silica ${ }^{[96,97]}$ and organosilica ${ }^{[98]}$ disclosed new opportunities, as their larger pore size could fit to macromolecules and nanoparticles. The rise of framework materials ${ }^{[9-101]}$ brought further variety of shapes and composition, structural flexibility, and systems that could undergo reversible changes upon light irradiation or mechanical pressure. ${ }^{[102-104]}$

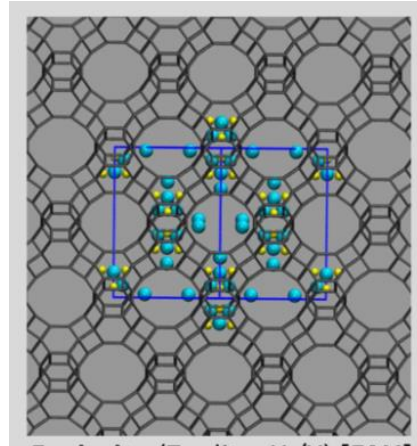

Faujasite (Zeolite $X / Y$ ) [FAU]

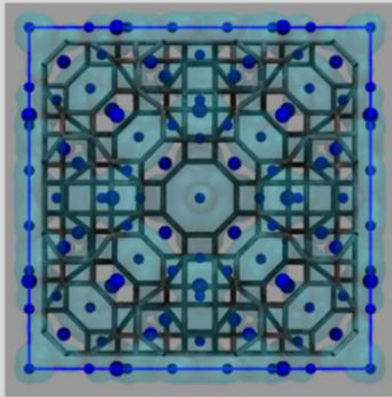

Paulingite [PAU]

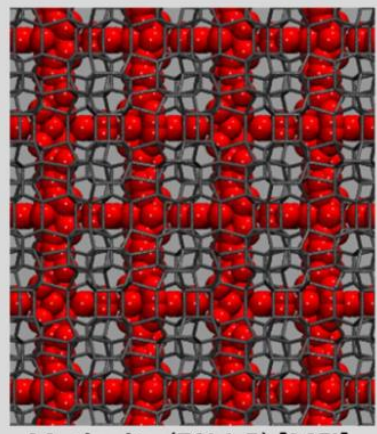

Mutinaite (ZSM-5) [MFI]

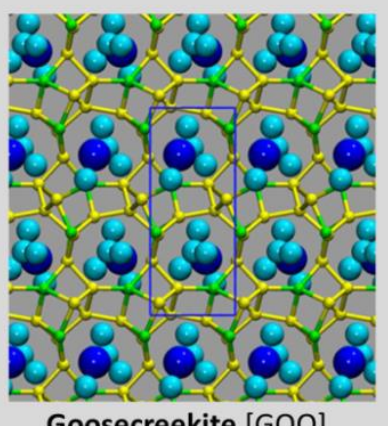

Figure 3. Structures of natural zeolites from X-ray diffraction. Top: Framework structures ( $\mathrm{Si}, \mathrm{Al}=$ grey sticks; oxygen omitted for clarity) of the natural zeolites faujasite ${ }^{[105]}$ (FAU) (blue solid lines indicate the unit cell) and mutinaite $^{[106]}(\mathrm{MFI})$, and of their synthetic counterparts (in parentheses). The colored balls are the water and cation sites (with fractional occupancy). [Illustration created with VMD, using the X-ray positions from Refs ${ }^{[105,106]}$. Framework structure $\left(\mathrm{Si}, \mathrm{Al}=\right.$ grey sticks) of natural zeolite paulingite $[\mathrm{PAU}]^{[107]}$ : the most complex mineral known to date. ${ }^{[108]}$ The colored balls are the cation sites (with fractional occupancy): the size of the ball is proportional to the occupancy of the site. The shaded light-blue regions represent the distribution of the water sites. The blue line represents the unit cell, containing 16 formula units $\left(\mathrm{Ca}_{2.57} \mathrm{~K}_{2.28} \mathrm{Ba}_{1.39} \mathrm{Na}_{0.38}\right)\left[\mathrm{Al}_{11.55} \mathrm{Si}_{30.59} \mathrm{O}_{84}\right] \cdot 27 \mathrm{H}_{2} \mathrm{O}$, with minor amounts of $\mathrm{Mg}$ $(<0.05), \mathrm{Sr}(<0.13), \mathrm{Mn}(<0.01)$, and $\mathrm{Fe}(<0.04)$. $^{[107]}$ [Illustration created with VMD, using the X-ray positions from Ref. ${ }^{[107]}$. Framework structure (yellow $=\mathrm{Si}$, green $=\mathrm{Al}$ ) and extraframework content $($ blue $=\mathrm{Ca}$, light blue $=$ water oxygen $)$ of goosecreekite [GOO], the only example of natural zeolite with a chiral framework [idealized formula: $\mathrm{CaAl}_{2} \mathrm{Si}_{6} \mathrm{O}_{16} \cdot 5 \mathrm{H}_{2} \mathrm{O}$ ]. [IIlustration created with VMD using the X-ray positions from Ref. ${ }^{[109]}$.

In the search of new frameworks, much effort was focused on mimicking functionalities of biological systems through biomimetic chemistry. ${ }^{[1]}$ However, mineralomimetics - the counterpart of biomimetics - has been successful as well. According to Iwamoto, this term indicates the chemistry of building mineral-like structures using materials that never give stable minerals in nature. ${ }^{[110-112]}$ For example, by recognizing the structural similarity between $\mathrm{Cd}(\mathrm{CN})_{4}$ and $\mathrm{SiO}_{4}$, the group realized clay-mimetic and zeolitemimetic cyanocadmate inclusion compounds through a crystalengineering strategy. ${ }^{[13]}$ 
The relationships between man-made and natural materials are profound. Structurally, zeolites can be viewed as composed by cation-templated "natural building units" ${ }^{[114,115]}$ Earlier syntheses of zeolites were inspired by minerals, ${ }^{[85]}$ as in the case of the industrially relevant zeolites $X$ and $Y,{ }^{[116]}$ isostructural with faujasite (Figure 3). ${ }^{[105]}$ Conversely, natural counterparts of synthetic zeolites were discovered a posteriori; a striking example is mutinaite (Figure 3), ${ }^{[106]}$ isostructural with ZSM-5, a catalytic landmark in petrochemistry. ${ }^{[84]}$ This interplay holds for clathrates as well: structure $\mathrm{H}$ hydrate was first created in laboratory, ${ }^{[117]}$ and then found in nature. ${ }^{[118]}$ Rare silica minerals similar to zeolites and isostructural with gas hydrates, the clathrasils, ${ }^{[119]}$ contain organic molecules - methane and other simple hydrocarbons ${ }^{[119]}$ - which recall the templates of synthetic zeolites (Figure 4). ${ }^{[120]}$ Some natural zeolites exhibit chirality ${ }^{[121]}$ (goosecreekite) or extraordinary structural complexity, ${ }^{[108]}$ like paulingite (Figure 3). A recently synthesized zeolite family ${ }^{[122]}$ was modeled upon paulingite, and key for the synthesis was the recognition that $\mathrm{Mg}$ and $\mathrm{Ca}$ cations - found in related zeolite minerals - could be used as auxiliary templating agents for the more complex members of the family. ${ }^{[123]}$

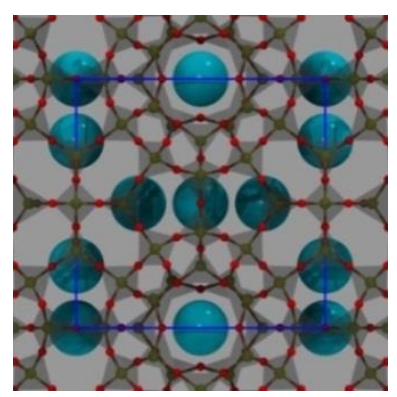

Figure 4. Methane naturally confined in a silica mineral. Structure of the allsilica mineral melanophlogite, historically recognized as the first clathrasil. ${ }^{[124]}$ The framework is illustrated using shaded grey tetrahedra superposed to a balland-stick representation $\left(\mathrm{O}=\mathrm{red}, \mathrm{Si}=\right.$ yellow). This mineral contains $\mathrm{CH}_{4}$ : the big light-blue spheres represent the experimentally determined $\mathrm{C}$ sites.[Illustration created with VMD, using the X-ray positions from Ref. ${ }^{[125]}$.

More fundamentally, insight on the interactions of simple species like methane, water, and zeolitic cations inside natural porous matrices is important to better understand the behavior of nanoobjects in confined spaces. For instance, natural zeolites contain structured water-ion networks ${ }^{[126,127]}$ which, under pressure, resist spectacularly or rearrange dynamically, ${ }^{[53]}$ and also the response of the framework is crucially influenced by the water-cation assemblies inside the cavities. ${ }^{[128]}$ Fundamental knowledge on the behavior of such aggregates at high pressure could help increasing the stability range of technologically relevant hostguest materials. For these reasons, care will be taken to compare artificially created composites with natural systems showing either relevant similarities or unexpected relationships.

\section{Characterization techniques}

Many powerful techniques are employed to study host-guest compounds. Among widespread methods, some are particularly useful to investigate guest species and their environment.

Structural determination is typically based on Bragg diffraction, which probes long-range order in periodic solids. However, the presence of guests usually lowers the symmetry of the host, hindering structural characterization of the confined species with standard crystallographic methods. ${ }^{[129]}$ In general, when deviation from periodicity (i.e., disorder) is present, only a configurational average over all possible disordered states is obtained. Such average structure appears to be more symmetrical that the actual material, because all disorder is reflected back into one unit cell, losing thus important structural insight. As the unit cell of a disordered material is actually the entire crystal, a proper structural description would require also short-range parameters like geometry of the local environment and next-nearest neighbors. Total scattering measurements do not rely on periodicity and give insight into short/medium range correlations (e.g hydrogen bonds) in disordered materials, which typically escape standard diffraction. The Fourier transform of the total scattering structure factor is the pair distribution function (PDF), a weighted sum of probabilities to find atoms of one type at a given separation from atoms of another type. Modern crystallographic techniques can integrate single crystal, powder, or neutron diffraction data with PDF analyses. ${ }^{[33,130,131]}$ Such advances have fostered structural studies on disordered phases, (such as the recently reported liquid MOFs), ${ }^{[132]}$ dye-zeolite materials, ${ }^{[133]}$ or catalysts under industrial conditions, unveiling, for example, the interaction of water with SAPO-34 acidic sites and their environment at high temperature $\left(700^{\circ} \mathrm{C}\right) .{ }^{[134]}$

Complex materials like inclusion compounds normally require multitechnique characterization approaches. Regarding structural analyses, the combination of diffraction and magic angle spinning (MAS) solid-state NMR spectroscopy (SS-NMR) is an excellent strategy, termed "NMR crystallography" [135] (see CrystEngComm, 2013, 15, 8589 for a themed issue). Bragg diffraction and SSNMR are complementary: whereas diffraction provides an average overview of the long-range structure, NMR does not rely on periodicity and captures detailed "short-range" information on the environment of specific nuclei, including their dynamics and local order/disorder. Because different local geometries imply, in general, differences in the chemical shift, NMR data are widely used in the structural characterization of porous materials. For zeolites, one prototypical case is ${ }^{29} \mathrm{Si}$, sensitive to bond distances, angles, and next-nearest-neighbor environment: its usefulness in probing the $\mathrm{Si} / \mathrm{Al}$ ordering in zeolites has been long recognized. ${ }^{[136]}$ Besides ${ }^{29} \mathrm{Si},{ }^{, 137]}$ also ${ }^{27} \mathrm{Al}$ plays a key role in topical issues, like characterization of zeolitic acid sites, ${ }^{[138]}$ or nucleation and growth processes, ${ }^{[139]}$ while ${ }^{33} \mathrm{P}^{[140]}$ is especially relevant for aluminophosphates. Many other nuclei $\left({ }^{1} \mathrm{H},{ }^{2} \mathrm{H},{ }^{13} \mathrm{C},{ }^{15} \mathrm{~N}\right.$, etc.) are largely exploited as well, e.g. for the study of MOF's ligands (see Ref. ${ }^{[135,141,142]}$ for reviews and Ref. ${ }^{[143]}$ for a recent multinuclear study of a formate MOF). Moreover, the Dynamic Nuclear Polarization approach allows to obtain high-quality ${ }^{13} \mathrm{C}$, ${ }^{1} \mathrm{H},{ }^{15} \mathrm{~N}$ SS-NMR spectra with natural isotopic abundance. ${ }^{[144]}$

With NMR spectroscopy it is also possible to probe the uniformity and the connectivity of the pores. ${ }^{129} \mathrm{Xe}$ chemical shift is a good probe of the inner pore surfaces, because it spans a wide range, and is very sensitive to the shape and size of the void..$^{[145]}{ }^{129} \mathrm{Xe}$ NMR has been applied to gas hydrates ${ }^{[146]}$ and zeolites, ${ }^{[147]}$ but also to MOF's, ${ }^{[148]}$ organic zeolite analogs ${ }^{[149]}$ and mesoporous materials. ${ }^{[150]}$ In the latter case, for example, the ${ }^{129} \mathrm{Xe}$ chemical shift can be correlated with the pore diameter. ${ }^{[151]}$ With optical pumping techniques it is possible to produce hyperpolarized xenon which allows for a large increase in signal intensity. This high sensitivity has been exploited to study the distribution of the ibuprofen drug in functionalized mesoporous silicas. ${ }^{[152]}$

Also the dynamic behavior of the guests can be probed by solidstate NMR spectroscopy. In this respect, variable-temperature ${ }^{2} \mathrm{H}$ NMR experiments are well-established tools: for instance, they 
have recently allowed to characterize the dynamics of ethylene bound to $\mathrm{Ag}^{+}$site in $\mathrm{Ag} / \mathrm{H}-\mathrm{ZSM}-5$ zeolite, highlighting two internal rotations of the guest about its symmetry axes. ${ }^{[153]}$

Also vibrational spectroscopies (principally, IR and Raman) are prime techniques for porous materials. ${ }^{[154]}$ In particular, IR spectroscopy of adsorbed probe molecules, like $\mathrm{NH}_{3}{ }^{[155]}$ or $\mathrm{CO},{ }^{[156-158]}$ is very sensitive to the environment of the absorption site, hence a powerful method to study, e.g., Brønsted or Lewis acid centers in zeolitic frameworks. More exotic approaches, such as photoacoustic IR, enable a precise discrimination of linkers in MOF via the sound produced upon IR irradiation. ${ }^{[159]}$ Raman spectroscopy is widely used to study the orientation of guest species at various temperature/pressure conditions (see e.g. examples in chapters 6 and 8). Coherent anti-Stokes Raman scattering spectromicroscopy (CARS), ${ }^{[160]}$ is particularly sensitive: for example, it revealed the formation of head-to-tail chains of adsorbate molecules inside the pores of ZSM-5 zeolite. ${ }^{[161]}$

Most materials presented in this work are of interest for their optical properties. In this respect characterization is performed with UV-VIS-NIR and luminescence spectroscopies. ${ }^{[162]}$ The positions of the electronic bands are sensitive to the oxidation state and local coordination environment of the absorbing center. However, only the development of single-molecule techniques such as confocal fluorescence microscopy, has allowed gathering insight on the orientation of fluorescent molecules in 1 D zeolite channels. The electronic transition dipole moment of these molecules is normally aligned with their long molecular axis. ${ }^{[29]}$ The technique probes the orientation of the emission dipoles: for example, fluorescence detected upon irradiation with light polarized parallel to the crystal axis (which coincides with the zeolite channel axis) indicates that the molecules are aligned with the channel. ${ }^{[29,162]}$ While this method assumes a homogeneous angular distribution of the guests, such limitation is overcome by polarimetric two-photon fluorescence microscopy, which can quantify disorder at high concentrations of included dyes. ${ }^{[163]}$

In general single-molecule imaging/spectroscopy techniques have undergone an impressive advance in the latest years (see Refs. ${ }^{[164-167]}$ and special issue of ChemPhysChem 2012, 13, 8811095). Not only they give insight on the orientation of guests, but they also allow to visualize fine details of pore accessibility and connectivity - like the recent demonstration of pore-blocking during catalytic isomerization in zeolite ferrierite. ${ }^{[168]}$

EPR also provides valuable insight on local environment of paramagnetic species. Besides catalytically relevant transition metal centers, it has proved to be particularly effective in the study of zeolite-incorporated metal clusters (chapter 4). Particularly advantageous is also X-ray absorption spectroscopy (XAS), ${ }^{[169]}$ which is not limited to paramagnetic species. XAS measurements are element-specific, can be used for structural studies of both crystalline and amorphous materials, and may be even applied under "operando" conditions. ${ }^{[169]}$ A recent example is a XAS study on the oxidation state and active site geometry in $\mathrm{Ce}^{4+}$-MOFs demonstrating the redox activity of these materials, relevant for potential application in redox catalysis. ${ }^{[170]}$

Destructive techniques, like thermogravimetric (TGA) and differential scanning calorimetric (DSC) analyses, as well as mass spectrometry, are widely employed for qualitative and quantitative elucidation of the guest content of inclusion compounds, and are particularly useful when combined with non-destructive analyses as the above-mentioned ones.

Finally, the use of computational approaches such as quantum chemical and molecular dynamics calculations enables to capture structural and dynamical features otherwise difficult to gather from experiments. Details on the application of computational methods to porous materials can be found in a recent comprehensive review, ${ }^{[171]}$ technical books ${ }^{[172]}$ and MOF-specific accounts. ${ }^{[173-175]}$

\section{Empty space architectures}

Porous materials may be classified, according to IUPAC, in three groups: microporous (pore diameters below $2 \mathrm{~nm}$ ), mesoporous (pore diameters between 2 and $50 \mathrm{~nm}$ ), and macroporous (pore diameters over $50 \mathrm{~nm}$ ). Beside those herein discussed, other classes of porous solids, like organic cages ${ }^{[176]}$ polymers, ${ }^{[177]}$ and all-inorganic polyoxometalates, ${ }^{[178]}$ have further widened the possible functionalities making it hard to select the best material for a given application. ${ }^{[179]}$ Although regular porous solids have ordered pore networks, the walls may be crystalline (zeolites), amorphous (mesoporous silica) or broadly periodic (organosilica). As discussed by various reports, ${ }^{[44,180-184]}$ and a recent review, ${ }^{[185]}$ the guest species undergo complex adsorption/diffusion processes before reaching an equilibrium arrangement inside the pores. Such organization of the guests can then be extended to the macroscale using assembly approaches in the case of colloidal particles/microcrystals. ${ }^{[186,187]}$ Other strategies ${ }^{[188-190]}$ imply the attachment of the microcrystal on a support. In this case, a key role is played by the terminations of the porous crystals, still little explored at atomistic detail. ${ }^{[191]}$ A deep knowledge of these regions is fundamental for both the guest inclusion process and the final utilization of host-guest materials in functional devices. ${ }^{[36]}$

\subsection{Zeolites}

Zeolites are microporous crystalline materials mainly made up by aluminum, silicon and oxygen. ${ }^{[58,192]}$ While natural zeolites are important in earth science and mineralogy, ${ }^{[126]}$ synthetic zeolites are ubiquitous in industry and technology. ${ }^{[83,188]}$ Owing to their thermal stability and selectivity, zeolites have been adopted since early 1960s in a wealth of industrial processes involving catalysis, adsorption and ionic exchange, and are key components of detergents and desiccants. ${ }^{[193]}$ Their potential as host matrices for nanosystems ${ }^{[41]}$ or building blocks for energy harvesting devices has been recognized for long time. ${ }^{[14,29]}$ The primary building units of zeolite frameworks are corner-sharing tetrahedra $\mathrm{TO}_{4}$, where $\mathrm{T}$ indicates an atom - normally $\mathrm{Si}$ or $\mathrm{Al}$ - bonded to four oxygens. The frameworks define architectures of channels and cages of nanometer size, which host various extraframework species molecules, ions, nanostructures or supramolecular aggregates according to the shape and size of the pores. ${ }^{[126]}$

\subsubsection{Structural aspects}

To date, the database of the International Zeolite Association $\left(\right.$ IZA) ${ }^{[60]}$ contains 235 zeolite structures (each identified by a three-letter code) which may be classified according to the size of the pore openings (i.e., the number of $\mathrm{T}$ atoms), or the number of dimensions defined by the pores. ${ }^{[58,194]}$ Other structural descriptors provide information on the geometry of the pores, ${ }^{[195]}$ the building blocks of the framework, ${ }^{[196]}$ or the environment of a T atom, ${ }^{[197]}$ as nicely documented by a themed review. ${ }^{[198]}$

The high number of zeolite framework types (or "topologies") implies a wide variety of cages and channels, with different geometry and size. The diameters of pore openings typically range between 0.3 to $0.8 \mathrm{~nm}$, while those of the inner cavities are larger, namely from 0.5 to $1.3 \mathrm{~nm} .{ }^{[58]}$ 


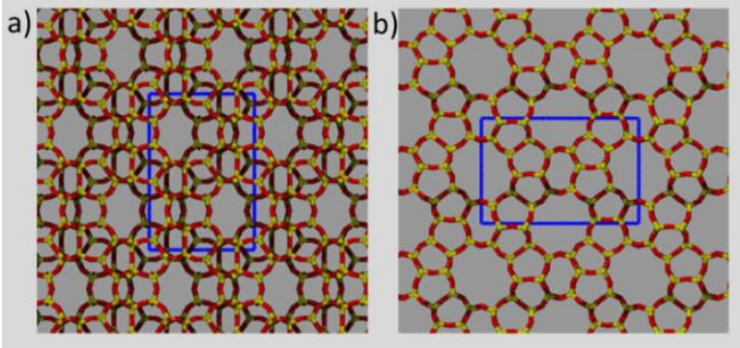

Figure 5. The framework structure of silicalite (MFI) with a 3-D channel system projected in the a) bc plane; b) ac plane. Yellow $=\mathrm{Si}$, red $=\mathrm{O}$, blue line $=$ unit cell. [Illustrations created with VMD using X-ray data from Ref. $\left.{ }^{[58,60]}\right]$

Zeolitic channels form one-dimensional, two-dimensional, and three-dimensional arrays of void space. In one-dimensional (1-D) channel systems, the channels run parallel to one direction and do not intersect. Two-dimensional (2-D) channels cross each other: intersections, though beneficial in catalysis, ${ }^{[199]}$ make the production of sequential arrangements of guests difficult to control. ${ }^{[36]}$ The same holds for 3-D channels (an example is the industrially relevant MFI framework of silicalite, Figure 5).

Zeolites can be also produced in a two-dimensional form, ${ }^{[200]}$ - a feature which opens new routes to the surface confinement of nanosized species. They are synthesized as nanosheets or ultrathin films deposited on metal oxides ${ }^{[201]}$ and metal surfaces. ${ }^{[202]}$ Other intriguing variants, such as layered ${ }^{[203,204]}$ or hierarchical macroporous zeolites[205-207], have been invented in the latest years. These advances have allowed to "escape from the $10 \AA$ prison" of zeolite nanospaces - as aptly termed by Ozin ${ }^{[208]}$ broadening thus the scopes of these materials as organizing scaffolds for diverse nanospecies.

\subsubsection{Chemical aspects}

All-silica zeolites, often referred to as Zeosils, are formed by $\mathrm{Si}$ tetrahedra only, and their framework is neutral. Traditional zeolites are aluminosilicates: each Al brings formally one negative charge to the framework. Such a charge is balanced by extraframework ions, mainly alkaline and alkaline-earth cations, hosted in the cavities.

Although often regarded as perfect, symmetric crystals, zeolites do contain defects. The framework is occasionally interrupted by hydroxyl groups occupying tetrahedral apexes ${ }^{[194]}$ or by cracks, as recently revealed by confocal fluorescence microscopy on crystals of ZSM-5, BEA ${ }^{[209]}$ and FER. ${ }^{[210]}$ Also, homogeneous crystals are actually constituted by complex crystalline intergrowths. ${ }^{[211]}$ In general, randomly distributed defects obstacle the fabrication of regular arrays of confined species.

Another important issue is the so-called structural disorder, which is related to the repartition of the Al atoms over the T-sites of the framework, ${ }^{[194]}$ and affects the positioning of charge-balancing cations. The distribution of $\mathrm{Al}$ atoms over the zeolite sites is difficult to determine by $\mathrm{X}$-rays because $\mathrm{Al}$ and $\mathrm{Si}$ have very close scattering factors. ${ }^{[194,198]}$ The different effects of $\mathrm{SiO}_{4}$ vs. $\mathrm{AlO}_{4}$ neighbors on ${ }^{29} \mathrm{Si}$ and ${ }^{27} \mathrm{Al}$ resonances make SS-NMR the technique of choice for determining Si-Al site occupancies and structural disorder, ${ }^{[135,136]}$ especially when combined with modeling. By ${ }^{27} \mathrm{Al}$ SS-NMR spectroscopy and DFT calculations, for example, Sklenak et al demonstrated that the repartition of $\mathrm{Al}$ sites over crystallographic positions in differently synthesized ZSM-5 samples depends on the synthesis conditions. ${ }^{[212]}$ In silico, the $\mathrm{Si} / \mathrm{Al}$ problem is typically tackled by calculating the energy of model structures with different distribution of these atoms on the crystallographic sites ${ }^{[213-215]}$ (same strategy holds also for the distribution of $\mathrm{Si}$ in silicoaluminophosphates ${ }^{[216,217]}$ or heteroatoms like $\mathrm{Ti}$ in zeolite catalysts, e.g. TS-1 ${ }^{[218-221]}$.

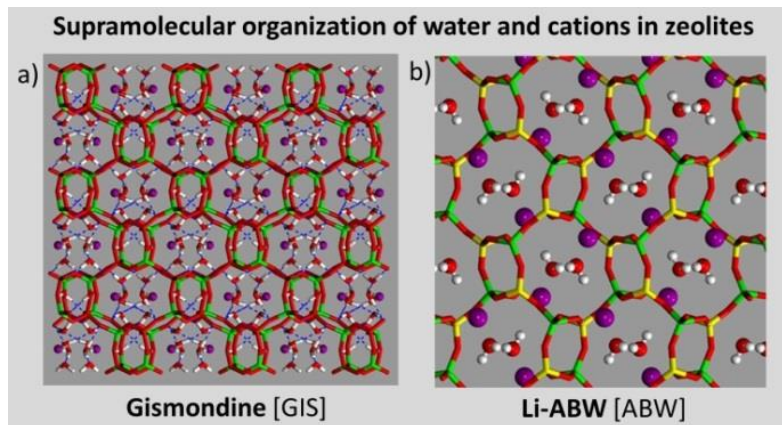

Figure 6. Organization of extraframework species a) The supramolecular network of calcium cations and water in natural zeolite gismondine (GIS, 3-D channel system). Colors: purple $=\mathrm{Ca}$, green $=\mathrm{Al}$, dark red $=\mathrm{Si}$, red $=\mathrm{O}$, white $=\mathrm{H}$; blue dashed line=hydrogen bonds. b) Water molecules in single file in synthetic zeolite Li-ABW (ABW, 1-D channel system): hydrogen bonds with framework oxygens are very weak. Colors: purple $=\mathrm{Li}$, green $=\mathrm{Al}$, yellow $=\mathrm{Si}$, red $=\mathrm{O}$, white $=\mathrm{H}$. [Created with VMD, using the X-ray positions from Refs. ${ }^{[222,223]}$

Along with cations, the pores normally contain water molecules. The interactions of these species with framework oxygens form a complex network whose structure and connectivity depends on the size, geometry, and composition of the zeolite cavity, besides the nature and number of encaged species. For example, while in gismondine (3D-channels) water molecules are hydrogen-bonded both to the framework and with each other (Figure 6a), in the tight 1D-channels of Li-ABW waters prefer to bind with themselves, forming linear chains along the channel axis (Figure 6b).

Water plays a prominent part in governing supramolecular organization inside the pores. Zeolites undergo dehydration below $400^{\circ} \mathrm{C}$ and the process is normally reversible. ${ }^{[194]}$ This ability of reversibly losing and gaining water via dehydration / rehydration cycles is useful for tuning the orientation of dye molecules in zeolite channels (see Chapter 7).

Extraframework cations, being non-covalently bonded to the framework, can be exchanged by washing with a concentrated solution of a desired cation, thus making zeolites exceptional ionic sorbents or exchangers. ${ }^{[188]}$ If the cations are protons (which form covalent bonds with framework oxygens), acid sites are created, whose special reactivity enables to catalyze reactions of substantial relevance for oil industry like cracking of hydrocarbons, or methanol to gasoline processes. ${ }^{[224]}$ lonic exchange is also used in ship-in-a-bottle syntheses of zeolite-hosted luminescent Ag clusters, ${ }^{[39]}$ as well as for the incorporation of cationic dyes, an essential step in the production of artificial antenna systems and zeolite-based devices. ${ }^{[29,50]}$

New framework types, as well as materials with composition different from natural zeolites, are continuously synthesized. ${ }^{[188]}$ The possibility to replace $\mathrm{Si}$ and $\mathrm{Al}$ with different elements and to control the Si/Al ratio in the synthesis has produced zeolites with fine-tuned exchange, selectivity, and sorption properties exploited in industrial processes. ${ }^{[84,85]}$ For instance, the Si/Al ratio governs the hydrophilicity of the framework. Zeolites with greater $\mathrm{Al}$ content host a higher number of cations, adsorb water, and are used as ionic exchangers, while zeolites with a high Si/Al ratio, being more hydrophobic, have greater affinity for hydrocarbons and are used in petrochemistry. ${ }^{[84]}$ Besides industrial applications, ${ }^{[19,188]}$ the water affinity of the framework plays a key role in the supramolecular organization of guest species. 
Zeolites where atoms other than $\mathrm{Si}$ and $\mathrm{Al}$ occupy tetrahedral sites (zeotypes) are important for catalysis: ${ }^{[58]}$ for example, the acid strength of proton-containing zeolites ${ }^{[155,225-230]}$ can be tuned by replacing $\mathrm{Al}$ with $\mathrm{Ga}^{[231-234]}$ or $\mathrm{B} .{ }^{[235]}$ For instance, boron zeolites are less acidic than Al-ones due to the tendency of boron to be trigonal. ${ }^{[236-238]}$ The change of $B$ coordination from tetrahedral to trigonal leads to interruptions in the framework and is accompanied by a $10 \mathrm{ppm}$ downfield shift of ${ }^{11} \mathrm{~B}$ NMR signal, ${ }^{[237,239]}$ whereas tetrahedral boron geometry is recovered upon hydration. ${ }^{[240-242]}$ Such weaker Brønsted acidity of B-sites guarantees a high selectivity for ethylene from methane ${ }^{[243]}$ and is relevant for the environmentally friendly production of styrene. ${ }^{[244]}$ Aluminophosphates (ALPO) ${ }^{[245,246]}$ generally have neutral framework and large pore sizes, like in VPI-5. ${ }^{[86,87]}$ Titanium, tin, or zirconium impart Lewis acidity to zeolites, ${ }^{[247,248]}$ while Ge-containing frameworks exhibit higher flexibility, extra-large pores, and even chirality. ${ }^{[249-251]}$ Indeed, a peculiar structural weakness of Ge-frameworks under hydrolytic conditions is exploited in ADOR (assembly-disassemblyorganization-reassembly), a powerful approach for the synthesis of new zeotypes. ${ }^{[252]}$ Other elements have been incorporated in zeolite frameworks, ${ }^{[253]}$ yielding previously unseen zeolite types and fine-tuning of their chemical properties. ${ }^{[198,254]}$ Also, the fusion of organic functions with a crystalline framework has produced organic-inorganic aluminosilicates with open porosity, also known as "hybrid zeolites". ${ }^{255,256]}$ Such variety of shape and composition provides further scope to the confinement of guest species.

\subsubsection{Electronic aspects}

Zeolites are typically classified as insulators. The endeavor of imparting interesting electronic properties to their frameworks has a long history, ${ }^{[16]}$ involving both theoretical, ${ }^{[257]}$ and experimental efforts. ${ }^{[89,92]}$ Nitridosilicate and nitridophosphate zeolites, ${ }^{[93,258]}$ owing to their chemical stability, optical transparency, and electronic structure, can be suitable host matrices for $\mathrm{Eu}^{2+}$ doping, and have shown potential as high-performance white-light luminescence materials (Figure 7). ${ }^{259]}$ The recent realization of open-shell transition-metal nitridosilicates ${ }^{[260]}$ via post-synthetic cation exchange ${ }^{[261]}$ may impart further appealing properties such as magnetism - to this interesting class of zeotypes.

Also the titanosilicate ETS-10[88] is a remarkable material, featuring a low-dimensionality nanosystem encaged into a zeolite framework. Symmetrically distributed $\mathrm{TiO}_{3}{ }^{2-}$ wires occupy zeolite nanochannels, running along perpendicular directions (Figure 8). ${ }^{[262,263]}$ Significantly, the wires displayed neat quantum size effects - band gap dependency on the wire length - and very promising optical properties. ${ }^{[264,265]}$
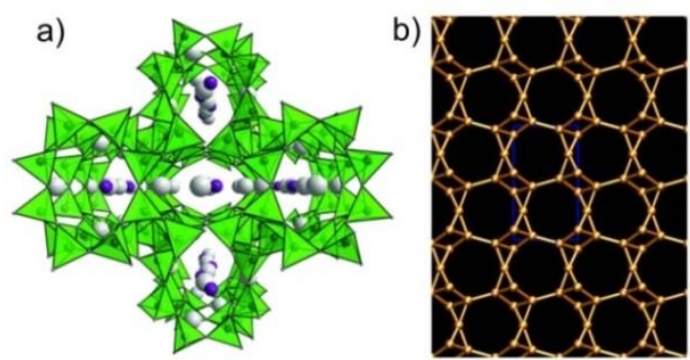

Figure 7. A host matrix for high-performance luminescence materials: a) the nitridophosphate zeolite $\mathrm{Ba}_{3} \mathrm{P}_{5} \mathrm{~N}_{10} \mathrm{Br}^{[259]} \quad\left(\mathrm{Br}=\right.$ purple, $\mathrm{Ba}=$ gray, $\mathrm{PN} \mathrm{N}_{4}$ tetrahedra $=$ green, JOZ framework type ${ }^{[58,60]}$. It exhibits excellent white-light emitting properties upon $\mathrm{Eu}^{2+}$ doping. [Reproduced by permission from Wiley$\mathrm{VCH} .{ }^{[259]}$ b) JOZ framework structure [Image realized with VMD using the X-ray positions from Ref. $\left.{ }^{[58,60]}\right]$

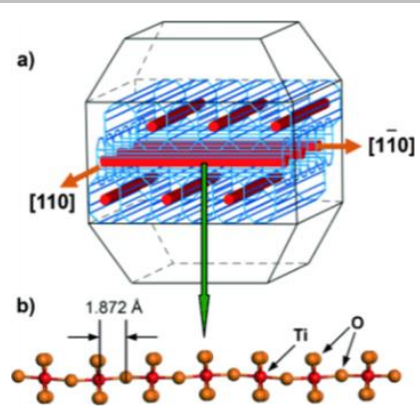

Figure 8: Confined $\mathrm{TiO}_{3}{ }^{2-}$ quantum wires. The titanosilicate zeotype ETS-10 (a) hosts $\mathrm{TiO}_{3}{ }^{2-}$ quantum wires (b) in its channel system. The quantum wires have different length and are arranged into a symmetric pattern. [Reproduced by permission from Wiley- $\mathrm{VCH}$ ]. ${ }^{[263]}$

The fabrication of semiconductive frameworks has focused on the family of chalcogenide zeolites ${ }^{[91,253]}$ because of their promising behavior as electronic sensors. ${ }^{[90]}$ Formally, these materials are derived by replacing oxygen with chalcogen atom $(\mathrm{S} / \mathrm{Se} / \mathrm{Te})$ and $\mathrm{Si} / \mathrm{Al}$ with tetrahedrally coordinated ions such as $\mathrm{In} / \mathrm{Ga} / \mathrm{Sn} / \mathrm{Ge}$. Recently, Lin et al. fabricated an In-Se chalcogenide zeotype (CSZ-5-InSe) with n-type semiconductor properties, and an ordered distribution of interrupted sites. Such semiconducting zeolite behaved as an effective catalyst for the oxygen reduction reaction, with the In interrupted sites functioning as active centers. The material was then selectively doped with bismuth at the interruption sites, thus allowing for an atomically precise fine tuning of its electronic structure. ${ }^{[266]}$ In spite of the synthetic challenges posed by the fabrication of chalcogenide frameworks, this route seems thus worth pursuing - not only because the incorporation of semiconductor property into zeolite materials has proved to be possible, ${ }^{[266]}$ but also because the electronic properties of these materials are tunable from metal to insulator by chemical design, as predicted by a recent modeling study. ${ }^{[267]}$ Furthermore, beside their intrinsic relevance as transparent nanoporous semiconductors, ${ }^{[267]}$ these zeolite frameworks could also open totally new application prospects to the confinement of quantum dots, ${ }^{[267]}$ and chromophores (Figure 9). ${ }^{[268]}$ Specifically, $\mathrm{Hu}$ et al accomplished an energy transfer process with the participation of a chalcogenide-based zeolite (coded as RWY) acting as light-harvesting host. The acridine orange dye was encapsulated into the host, and contacted at the pore entrances with another dye (rhodamine B) too large to enter the pores. Upon UV light absorption by the host, energy was transferred to acridine orange, and then to rhodamine $B$ yielding visible light emission. ${ }^{[268]}$ The energy-funnel behavior of the RWY host was confirmed in a second experiment conducted with different dyes (proflavine and pyronine), which highlighted a significant increase of the energy transfer efficiency upon acidification and solvation of the guests. ${ }^{[269]}$ These studies candidate chalcogenide-based semiconductor zeolites as very interesting host materials for energy transfer processes and photocatalysis. ${ }^{[270]}$

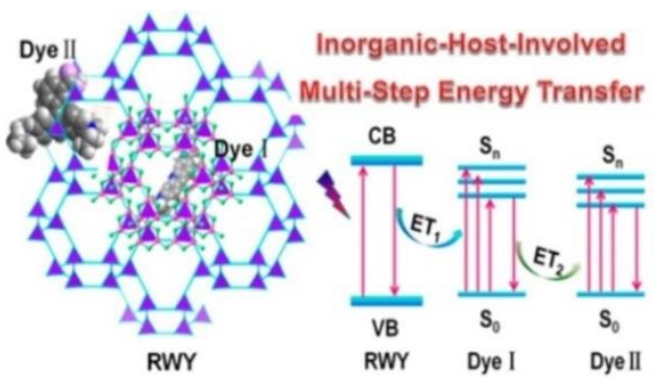


Figure 9. Chalcogenide zeolites incorporate semiconductive properties directly in zeolite frameworks. RWY is a chalcogenide zeolite that can host luminescent molecules and can be directly involved in energy transduction processes: here, the framework absorbs UV-light and transfers the excitation energy to dyes absorbing in the visible range. [Adapted with permission from Ref. ${ }^{[268]}$ Copyright 2015 American Chemical Society]

\subsection{Zero dimensional matrices: clathrasils and clathrates}

Some silicate minerals are structurally very close to zeolites. These polymorphs of $\mathrm{SiO}_{2}$, known as clathrasils, ${ }^{[271]}$ have cavities of sub-nanometer size like zeosils (all-silica zeolites), but with such small openings to prevent diffusion in and out of the pores; ${ }^{[119,124]}$ they might thus be pictured as inert hosts for nanomaterials of zero-dimensionality. Although melanophlogite (Figure 4) ${ }^{[125,272-274]}$ contains only very small molecules, like $\mathrm{CH}_{4}$, $\mathrm{CO}_{2}$ or $\mathrm{N}_{2},{ }^{[275,276]}$ other hydrocarbons are found in clathrasils with larger cavities: ${ }^{[119,277]}$ indeed, the recently discovered chibaite ${ }^{[278]}$ hosts methane, ethane, propane and isobutane. ${ }^{[119,277,278]}$

These minerals (Figure 10) are silica analogs of the well-known natural gas-storage media methane hydrates. ${ }^{[279,280]}$ Melanophlogite, for example, is isostructural with „structure I“ (sl) hydrates (Figure 10a), which have two kinds of cages: two pentagonal dodecahedra $\left[5^{12}\right]$ (with 12-sides) and six tetrakaidecahedra $\left[5^{12} 6^{2}\right]$ (with 14-sides) in the unit cell (figure 11a). Yet the multifaceted richness of clathrate hydrates goes well beyond the simple methane hydrate MEP topology - indeed, they encompass at least six framework types. ${ }^{[75]}$
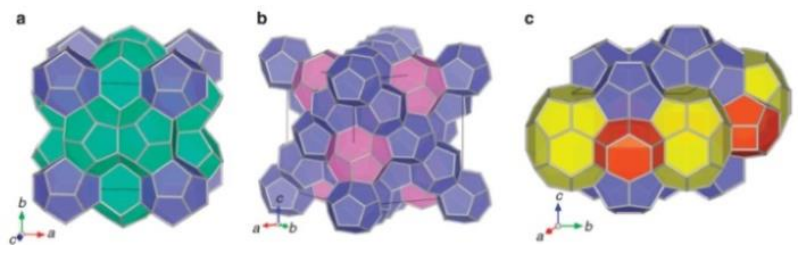

Figure 10. Three types of natural clathrasils (all-silica minerals): a) melanophlogite (MEP); b) chibaite (MTN) and c) mineral of type DOH as yet unnamed. These clathrasils are isostructural with hydrates of a) structure $I ; b$ structure II; c) structure $\mathrm{H}$, respectively. [Reproduced by permission from Macmillan Publishers Ltd.] a)

b)

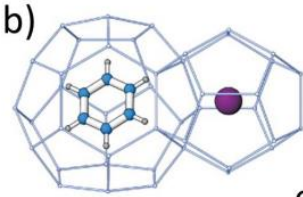

C)

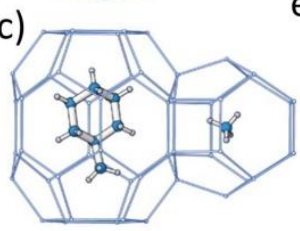

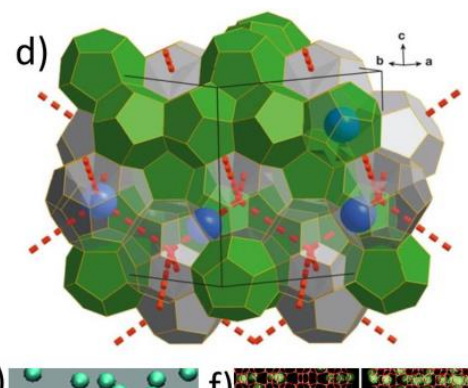

e)

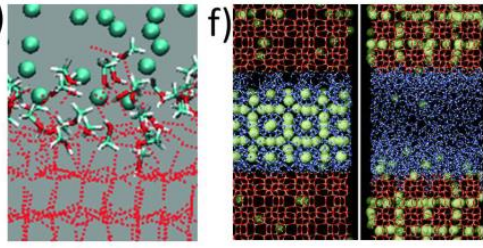

Figure 11. Clathrate hydrates a-c) Cages of the three main hydrate structures with encaged guests from single crystal $\mathrm{X}$-ray diffraction (only a single position for the guest species is shown in each cage): a) Ethane in the $\left[5^{12} 6^{2}\right]$ (left) and $\left[5^{12}\right]$ (right) cages of cubic structure I; b) cubic structure II, with benzene in the large $\left[5^{12} 6^{4}\right]$ cavity (left) and a Xe atom in the small $\left[5^{12}\right]$ cavity (right); c) Structure $H$, showing methylcyclohexane in the large $\left[5^{12} 6^{8}\right]$ cavity (left) and methane in the small $\left[4^{3} 5^{6} 6^{3}\right]$ cavity (right). [a-c) reprinted from Ref. ${ }^{[21]}$ with permission from Elsevier] d) Leaching of $\mathrm{Ne}$ atoms (blue) from sll clathrate structure: the atoms move only between large cages (grey) by transiting through six-membered rings of water molecules (the red dashed lines indicate the trajectory of $\mathrm{Ne}$ atoms). [Adapted by permission from Macmillan Publishers Ltd. $\left.{ }^{[282]}\right]$ e) Simulation snapshot illustrating the incipient formation of methane hydrate from frozen water/methanol (dots/sticks) solutions exposed to methane gas (cyan balls) at pressures of 30-125 bar and $\mathrm{T}=253 \mathrm{~K}$. [Adapted from Ref. [283] with permission from Wiley-VCH]. f) snapshot from MD simulations showing the conversion of a methane clathrate hydrate in contact with all-silica silicalite into methane-filled silicalite (red sticks=silicalite; blue sticks=water; green balls=methane) [Reproduced from Ref. ${ }^{[284]}$ with permission from the PCCP Owner Societies].

In general, clathrate hydrates are classic supramolecular systems formed by a framework of water, ${ }^{[75,279,285]}$ in which various ions or molecules are encaged (Figure 11a-c). Besides sl, there are two other important structures. One is a cubic symmetry phase, named (sll), of framework type MTN (Figure 10b): its unit cell contains sixteen $5^{12}$ cages and eight large hexakaidecahedral $\left[5^{12} 6^{4}\right]$ cavities (Figure $\left.11 \mathrm{~b}\right)$. The other phase, structure $\mathrm{H}(\mathrm{H}$ is for "hexagonal", framework type $\mathrm{DOH}$, figure 11c) comprises layers of $5^{12}$ cages alternated by layers with small $4^{3} 5^{6} 6^{3}$ cages and large barrel-shaped $5^{12} 6^{8}$ cages (Figure11c). ${ }^{[6]}$ Dimethyl ether forms a more exotic hydrate structure, ST hydrate, exhibiting a trigonal crystal structure. ${ }^{[286]}$ The remaining phases appear at elevated pressure - e.g. tetrahydrofuran hydrate, displaying space-filling $4^{4} 5^{4} 6^{6}$ cages, forms at $0.8 \mathrm{GPa}{ }^{[287]}$ Indeed, hydrates are often studied in this harsh regime, and for very good reasons: first of all, their pressure-induced stability increase. This allows hydrates to reach temperatures around $10^{\circ} \mathrm{C}$ (higher than ice melting point) at pressures of $0.1 \mathrm{GPa}^{[288,289]}$ Some hydrates, however, do not need high pressure to survive - like hydrogen sulfide hydrate, which can exist at $\sim 1$ atmosphere and 273K. ${ }^{[32]}$ Most hydrates crystallize in sl or sll structures, and the structure type is determined mainly - but not exclusively - by the size of the guests. Whereas $\mathrm{sl}$ is often formed with small molecules (e.g., methane), sll can contain larger guests (Figure 11b). Both large and small guests are needed for $\mathrm{sH}$ to form (Figure 11c). Small molecules like $\mathrm{H}_{2}$, however, form sll hydrates. ${ }^{[290,291]}$ This happens because: i) sll has more small cages than sl; ii) the large $5^{12} 6^{4}$ cages can accommodate multiple $\mathrm{H}_{2}$ molecules.

Recall that clathrate hydrates are prototypical non-stoichiometric compounds: it is not necessary for all cages to be occupied. In the ethane hydrate of Figure 11a, the large cage is fully occupied while the small cage was found to be nearly empty ( $5 \%$ occupancy) giving a composition of $\mathrm{C}_{2} \mathrm{H}_{6}{ }^{*} 7.52(1) \mathrm{H}_{2} \mathrm{O}$. ${ }^{[281]}$ The cage occupancies are thus very different from $\mathrm{CH}_{4}$ hydrate, where all cages are strongly occupied (large: 100\%, small: $90 \%),{ }^{[76]}$ and $\mathrm{CO}_{2}$ hydrate, where the $100(2) \%$ and $71(3) \%$ occupancies for large and small cages determined by singlecrystal X-rays yielded a hydration number of 6.20(15). ${ }^{[292]}$ Hence, by single crystal $\mathrm{X}$-ray (or neutron) diffraction it is possible to elucidate the composition of these phases and their structure, provided that a proper analysis of guest disorder is performed. [281] For example, the ethane hydrate of Figure 11a is disordered over 8 symmetry equivalent positions: their analysis indicated that the guests are not in the center of the cages, rather they stay close to the walls. ${ }^{[281]}$ The same holds for the benzene molecules in Figure 11b. This happens because close guest-framework contacts allow to maximize the weak intermolecular forces underlying the stability of hydrates - which is traditionally ascribed to guest-host steric repulsion from hydrophobic species. ${ }^{[293]}$ Nevertheless, 
some hydrates can also display attractive interactions between water framework and guests - such as hydrogen- and halogenbonding. In particular the latter ones, which were studied since the 1950 s by the Hassel group, ${ }^{[294]}$ have witnessed a growing interest in the last 20 years in view of their usefulness in crystal engineering (see e.g. Refs ${ }^{[295-297]}$ for reviews). A halogen bond is defined by IUPAC as "a net attractive interaction between an electrophilic region associated with a halogen atom in a molecular entity and a nucleophilic region in another, or the same, molecular entity". ${ }^{208]}$ These interactions - arising from halogens' affinity for the lone pairs of Lewis bases - are ubiquitous in supramolecular chemistry and may be also responsible of the unexpected structures found for dihalogen clathrates. Single crystal X-ray experiments ${ }^{[299]}$ showed that while $\mathrm{Cl}_{2}$ forms a standard sl hydrate with a surprising $32.5 \%$ occupancy of the small cages, $\mathrm{Br}_{2}$ hydrate crystallizes into a tetragonal slll phase, first proposed in $1963,{ }^{[300]}$ but extremely rare among hydrates. Also, the mixed $\mathrm{Cl}_{2}-\mathrm{Br}_{2}$ hydrate is of sl-type, but the larger $\mathrm{Br}_{2}$ molecules in the $\mathrm{Cl}_{2}$ hydrate lattice dramatically deform the cubic structure. ${ }^{[299]}$ Based solely on van-der-Waals radii, these molecules would never fit into such hydrate cages, indicating thus the presence of attractive interactions stronger than pure dispersion forces. The short $\mathrm{O} \cdots \mathrm{Cl}$ and $\mathrm{O} \cdots \mathrm{Br}$ distances obtained from refinement were then ascribed to halogen bonding. ${ }^{[299]}$ Actually, because the water oxygen lone pairs are already involved in the hydrogen bonds forming the clathrate lattice, the interactions of $\mathrm{Cl}_{2}$ and $\mathrm{Br}_{2}$ with water in hydrates should be somewhat different from classic halogen bonds - for instance, they lack the characteristic quasi-linearity. As evidenced by recent theoretical studies, ${ }^{[301-303]}$ much effort is now being directed to capture the nature of such unconventional halogen interaction.

Hydrates. In spite of innumerable studies, these curious forms of ice still remain amazingly surprising. It was thought that they must always contain molecules in order to avoid collapse. Yet such rule was broken by a spectacular experiment which accomplished the emptying of a neon hydrate, bringing thus to light the seventeenth phase of ice. ${ }^{[282]}$ This achievement was believed to be impossible, because the stability of hydrates relies on the weak interactions between the water framework and its guests. Inspired by the discovery of empty Ge-clathrates, ${ }^{[304]}$ and by previous work suggesting diffusion of $\mathrm{H}_{2}{ }^{[305,306]}$ or $\mathrm{Ne}^{[290]}$ through the clathrate lattice, Falenty et al. subjected neon hydrate to continuous vacuum pumping. After 5 days, all guests were gone, and the empty hydrate was studied by neutron diffraction. The empty clathrate was found to resist up to temperatures of $\sim 145 \mathrm{~K}$ before decomposing and to undergo a considerable enlargement of its framework with respect to the guest-containing hydrate. ${ }^{[282]}$ The proposed emptying mechanism involves the passage of $\mathrm{Ne}$ between the large cages of the sll lattice (Figure 11d), while removal of $\mathrm{Ne}$ from the small cavities would require a "hole in the cage" - i.e. the presence of water vacancies. ${ }^{[307,308]}$

Such experiment, and the discovery of intracage diffusion (detected also for methane), ${ }^{[309]}$ underlined the relevance of kinetic effects in clathrate hydrates. Similar conclusions were drawn by a recent study on the formation of $\mathrm{CO}$ hydrate, which also pinpointed the role of guest-guest interactions in stabilizing double-CO occupancy of the large cage in structure sll. ${ }^{[310]}$ As already mentioned, the physico-chemical origin of hydrate structures is commonly ascribed to the solvation of hydrophobic guests, like those in Figure 11a-c. Many evidences show however that hydrate framework stability depends holistically on host-guest interactions: hence, on a balance of hydrophilic and hydrophobic components. In this respect, methanol is an archetypal case. To the gas companies, hydrates are a real nuisance: to prevent their formation in gas lines, they often add methanol, which acts as „inhibitor": in fact, its hydroxyl group has a disrupting effect on the hydrogen bond network of the hydrate lattice. In spite of this, alcohols can form hydrates when in presence of a help gas, like $\mathrm{CH}_{4}{ }^{\left[{ }^{[11]}\right.}$ Indeed, methane clathrate forms very rapidly by exposing frozen water-methanol mixtures to methane gas, using pressures from 125 bars at $253 \mathrm{~K} .{ }^{[283]}$ Simulation results suggested that the methanol hydroxyl group should be involved in a sort of unconventional "catalyst-role" during the formation of hydrates with hydrophobic guests from ice (Figure 11e).[283,312] This could explain the experimentally observed increase in the rate of methane hydrate formation (by up to two orders of magnitude) in presence of methanol.[283]

Also, methanol can be incorporated as a guest in clathrates having both water and ammonium fluoride in the lattice because the hydrogen bonding of methanol with $\mathrm{F}^{-}$or $\mathrm{NH}_{4}{ }^{+}$keeps the water network nearly unaltered. ${ }^{[313]}$ In this way, methanol can be used as a helper to obtain $\mathrm{NH}_{4} \mathrm{~F}$-hydrate lattices co-hosting various hydrophilic guests of potential interest in gas storage. ${ }^{[314]}$ Interestingly, like for dihalogens, ${ }^{[299]}$ attractive interactions (in this case, hydrogen bonding with $\mathrm{NH}_{4} \mathrm{~F}$ ) allow the guests to occupy cages smaller than their van der Waals dimensions. ${ }^{[314]}$

The discussed examples suggest the importance of deeply understanding formation and decomposition mechanisms of hydrates (see Ref. ${ }^{[315,316]}$ for reviews). To this aim, computational approaches are especially useful. For instance, confinement of methane hydrate between two slabs of hydrophobic silicalite was simulated by molecular dynamics, showing that the clathrate structure was destabilized by the interaction with surface silanol groups. ${ }^{[284]}$ At low temperature, the hydrate essentially maintains its structure, while increased thermal motion leads to destruction of the water cages, starting from the interface with the zeolite and proceeding layer-by-layer until complete hydrate decomposition (Figure 11f). Interestingly, the released methane is absorbed by the hydrophobic zeolite pores - an insight that might be useful in the quest of controlled methane extraction from natural hydrates. Among hydrates, the chemical variety of guest species is impressive. Also strong acids or bases can form clathrate-related structures, some of which endowed with sodalite topology (SOD). In this case, the guests are anions and the water lattice has a positive charge, because it incorporates the counter-ions. The clathrates formed by $\mathrm{HPF}_{6}, \mathrm{HAsF}_{6}$, and $\mathrm{HSbF}_{6}$ are especially representative. According by combined ${ }^{19} \mathrm{~F}$ NMR and powder $\mathrm{X}$ ray diffraction analyses, ${ }^{[317]}$ and single-crystal $\mathrm{X}$-ray data, ${ }^{[318]}$ $\mathrm{HPF}_{6}$ has composition $\mathrm{HPF}_{6} \cdot \mathrm{HF} \cdot 5 \mathrm{H}_{2} \mathrm{O}$, with disordered $\mathrm{PF}_{6}$ anions at the center of truncated cubo-octahedral cages, while the HF molecules randomly occupy one-sixth of the water positions in the SOD lattice. Interestingly, hydrates of tetramethylammonium hydroxide (and other strong bases) form a related structure with a tetramethylammonium cation as guest and a negatively charged water framework of SOD type ${ }^{[319]}$ Here, the analogy with natural zeolites is particularly striking, because also a sodalite-group mineral - tsaregorodtsevite, ${ }^{\left[{ }^{[20]}\right.}$ contains (as the only one known) a tetramethylammonium cation in its cages. By considering now silica clathrates, recall that the Latin word clatratus means that guests are trapped, but also protected, "by the bars of a grating". Indeed, clathrasils have an exceptional thermal stability at normal pressure. By this virtue, even highly reactive species - such as the methyl radical[321] and the $S_{2}$ molecule ${ }^{[322]}$ - can be characterized spectroscopically. ${ }^{[323]}$ For example, femtosecond pump-probe experiments captured the photodissociation and recombination reactions of iodine molecules inside the cages of decadodecasil-3R zeosil (DDR). ${ }^{[324]}$ Such experimental analyses provide relevant insight on the 
dynamics and reactivity of confined molecules - which might be also potentially useful in the realization of confined quantum dots. Silica clathrates can be synthesized in laboratory. ${ }^{[325]}$ Dodecasil3C (MTN type) was among the first ones to be produced. This is another case where the realization of the material ${ }^{[326]}$ came long before the discovery of the mineral, ${ }^{[278]}$ and the same happened to the corresponding hydrate (structure $\mathrm{H})^{[117,118]}$

Many framework compositions have been attained, leading to new materials for potential optoelectronic, thermoelectric ${ }^{[197]}$ or electrochemical applications, ${ }^{[280,327,328]}$ or catalytic use. ${ }^{[329]}$ Synthetic clathrates can be anionic, cationic, or neutral according to the formal charge of the lattice; apart few exceptions, ${ }^{[304,330-332]}$ they need to contain a guest in order to be stable.

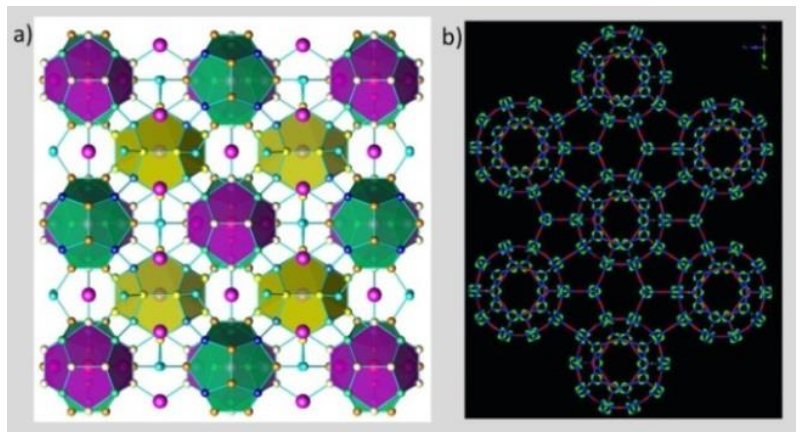

Figure 12. The peculiarity of inverse clathrates (a) is that guest species are negatively charged. This one is $\mathrm{Si}_{30.3(8)} \mathrm{P}_{15.7(8)} \mathrm{Se}_{7.930(3)}$ (ordered formula: $\mathrm{Si}_{32} \mathrm{P}_{14} \mathrm{Se}_{7}$ ) (MEP framework type): the Se guests are shown as big purple balls. [reprinted from Ref. ${ }^{[333]}$ with permission from Wiley- $\mathrm{VCH}$ ] $\boldsymbol{A}$ MOF clathrate of MTN topology (b) with large cages $\left(\sim 2.5 \mathrm{~nm}^{3}\right)$ The MOF $\left[\left\{\mathrm{Cd}\left(\mathrm{H}_{2} \mathrm{O}\right)_{3}\right\}_{34}\left(\mathrm{~N}_{4} \mathrm{C}_{6} \mathrm{H}_{12}\right)_{17}\right] \mathrm{Cl}_{68} \cdot 46 \mathrm{H}_{2} \mathrm{O} \cdot 68 \mathrm{DMF}, \mathrm{DMF}=\mathrm{N}$, N-dimethylformamide $]$ is viewed along the [110] direction. The six-membered ring openings measure $12.3 \times 13.1 \AA^{2}$ (between atom centers), while those of the five-membered rings are $10.4 \times 10.4 \AA^{2}$. Color codes: $\mathrm{Cd}=$ red, $\mathrm{N}=$ blue, $\mathrm{C}=$ green $(\mathrm{O}$ and $\mathrm{H}$ atoms are not shown). Reprinted from Ref. ${ }^{[334]}$ with permission from Wiley-VCH]

While negatively charged frameworks (the most abundant ones) trap cations, the positively charged lattices of "inverse" clathrates are particularly interesting because, unlike most zeolites, they encapsulate anions - normally halides, but also tellurium ${ }^{[35]}$ or selenium. ${ }^{[333]}$ For instance, the silicon-phosphorus framework of Figure 12 a traps Se in a partially covalent embrace, leading to a semiconductive material of potential thermoelectric use. ${ }^{[33]}$

In principle, clathrates formed by silicon, germanium, and $\operatorname{tin}^{[327,336,337]}$ should have neutral frameworks, like silica clathrates or clathrasils; however, charge transfer from the encaged species normally imparts them a formal negative charge. ${ }^{[338,339]}$ Hypothetical guest-free carbon clathrates have been studied for long time. ${ }^{[340,341]}$ In 1993, Nesper et al considered all zeolite and clathrate frameworks known at that time. They replaced each tetrahedral unit with a carbon atom and performed structural optimization and first-principles (Car-Parrinello) ${ }^{[342]}$ molecular dynamics on those structures. Interestingly, calculations predicted that the modification derived from zeolite ZSM-39 ( $\left.\mathrm{C}_{\text {MTN }}\right)$ and melanophlogite $\left(\mathrm{C}_{\text {MEP }}\right)$ were the most stable hypothetical carbon allotropes after graphite and diamond. ${ }^{[340]}$ Recently, a similar search was performed on $\approx 600000$ networks, ${ }^{[341]}$ yet no such material has been so far synthesized. Other theoretical investigations suggest that mixed carbon-boron clathrate frameworks filled with strontium might be stable..$^{[343]}$

In general, the confinement in clathrate cages allows for the guest species to be studied with multitechnique approaches aimed at a deeper understanding of their interactions with the framework.
This feature is common to small-caged zeolites such as sodalite (SOD) or ZSM-39 (MTN). ${ }^{[271,344]}$ Also many MOFs have clathrate topologies. For instance, both MIL-100 ${ }^{[345]}$ and the Cd-MOF in Figure $12 b^{[334]}$ are of MTN-type, featuring tetrahedral metalorganic building blocks and very large cages. ${ }^{[334,345]}$

Hence, clathrates bear very close links with other classes of porous materials, which are likely to be further enforced by the rising research area of confined hydrates. ${ }^{[346]}$

\subsection{Mesoporous silica and organosilica hybrids}

Compared to zeolites, mesoporous materials offer the possibility of accommodating larger objects inside their cavities, and may be either crystalline (long-range order of the pores) - or amorphous.

3.3.1. Mesoporous silica materials

Regular mesoporous silicas ${ }^{[347,348]}$ and metal oxides, ${ }^{[349]}$ as well as their hybrid organic-inorganic variants, ${ }^{[350-353]}$ occupy a leading place in applications (see e.g. Chem. Soc. Rev. 2013, Issue 9). 3D-mesopore systems are suitable for catalysis because they are less plagued by pore-blocking, while 1D systems are often preferred for encapsulating biomolecules, ${ }^{[354-356]}$ drugs, ${ }^{[357]}$ and confined nanostructures, such 1D-metal nanowires. ${ }^{[349,358,359]}$

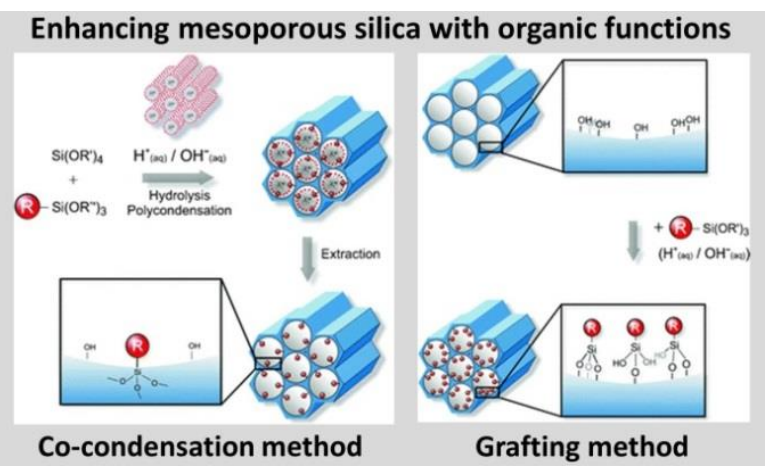

Figure 13. Imparting functionality to mesoporous silica. In the co condensation (“direct synthesis") approach, a trialkoxyorganosilane R-Si(OR') is added to the precursors. $R=$ organic functionality incorporated during the synthesis. The grafting method (post-synthesis) involves the condensation of e.g. trialkoxyorganosilane $\mathrm{R}-\mathrm{Si}\left(\mathrm{OR}^{\prime}\right)_{3}$ with silanol groups on the silica surface. [Adapted from Ref. ${ }^{[360]}$ with permission from The Royal Society of Chemistry]

MCM-41 and SBA-15 have non-intersecting one-dimensional channels with long-range hexagonal order and well-defined diameters (Figure 13). The channel diameter is determined essentially by the template, generally removed by calcination after polymerization. ${ }^{[347]}$ By a judicious choice of template and synthesis conditions, it is possible to tune the size of the channel according to the size of specific pre-selected guests. ${ }^{[20,347]}$ Also, mesoporous silicas can incorporate additional functionalities into their matrix via 1) "one-pot" synthesis, i.e. by co-condensation of a functional organosilane with the silica precursor, or 2) "postcondensation", which consists in the binding ("grafting") of the functional organosilane to the walls of the mesoporous material, usually by impregnation or through the vapor phase (Figure 13). ${ }^{[350,360-362]}$ The presence of a great number of surface silanols on the inner pore walls favors post-synthesis functionalization approaches. These are particularly convenient for optimizing the interaction with biomolecules, ${ }^{[363]}$ or the positioning of encapsulated chromophores in artificial antenna system. ${ }^{\text {[64-366] }}$ Post-synthesis strategies have also allowed to functionalize mesoporous silica nanospheres with molecular machines, creating intriguing devices for controlled release of drugs. ${ }^{[367-369]}$ 
For example, by attaching bistable rotaxane molecules to the pore entrances, Zing et al. realized molecular valves which could reversibly open or close the channel entrance commanded by redox inputs, ${ }^{[370]}$ or sequentially load and release molecules of different size (Figure 14). ${ }^{[371]}$

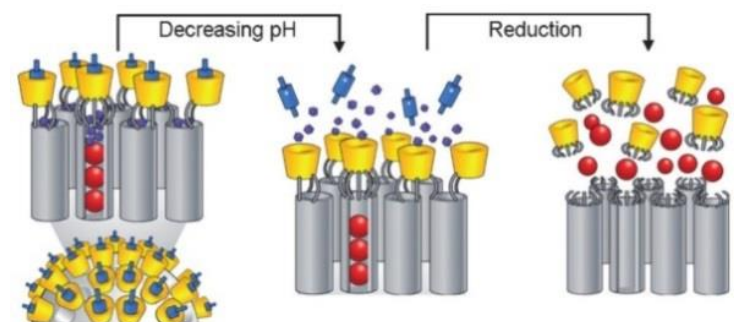

Figure 14. A sophisticated mesoporous silica-based drug delivery system. Pore entrances are -functionalized so as to release two drugs of different size (blue and red balls) one at a time upon the controlled action of different stimulations. The image illustrates schematically the two-step release process: the smaller molecule (blue balls) is released by lowering the $\mathrm{pH}$, and the larger one by a chemical stimulation (in this case, by addition of a reducing agent). [Reprinted with permission from Wiley- $\mathrm{VCH}$. $]^{[371]}$

\subsubsection{Mesoporous organosilica materials}

The invention of periodic organosilica ${ }^{[98,372-374]}$ offered the exciting opportunity to do "chemistry of the channel walls"[375] of mesoporous materials. Differently from the silica counterparts, the pore walls of mesoporous hybrid organic-inorganic systems are constituted by organosilanes, where silicon atoms are bridged by organic groups. ${ }^{[98,372,375]}$ The organic functionality is, thus, "buried" in the walls as in co-condensed functional mesoporous silica, but in higher concentration and with a uniform distribution (Figure 15). ${ }^{[350]}$ An early example that vividly pictures the "chemistry of the channel walls" in action, is mesoporous methylene silica. ${ }^{[375]}$ The methylene group of the framework could be converted upon chemical stimulation into methyl group grafted on the channel walls, demonstrating the potential of these systems to exhibit a responsive, stimuli-driven behavior ${ }^{[375]}$

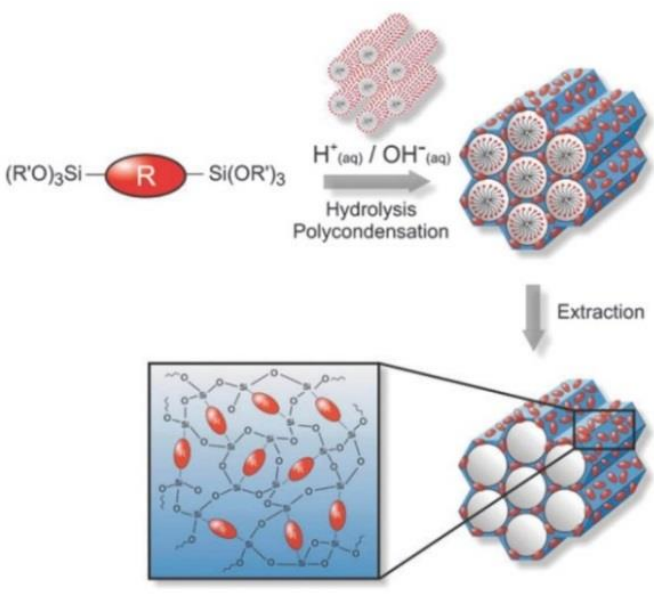

Figure 15. Schematic representation of the fabrication of PMOs starting from bis-silylated organic bridging units $R$. [Reproduced from Ref. ${ }^{[360]}$ with permission from The Royal Society of Chemistry]
PMO-based light-harvesting systems
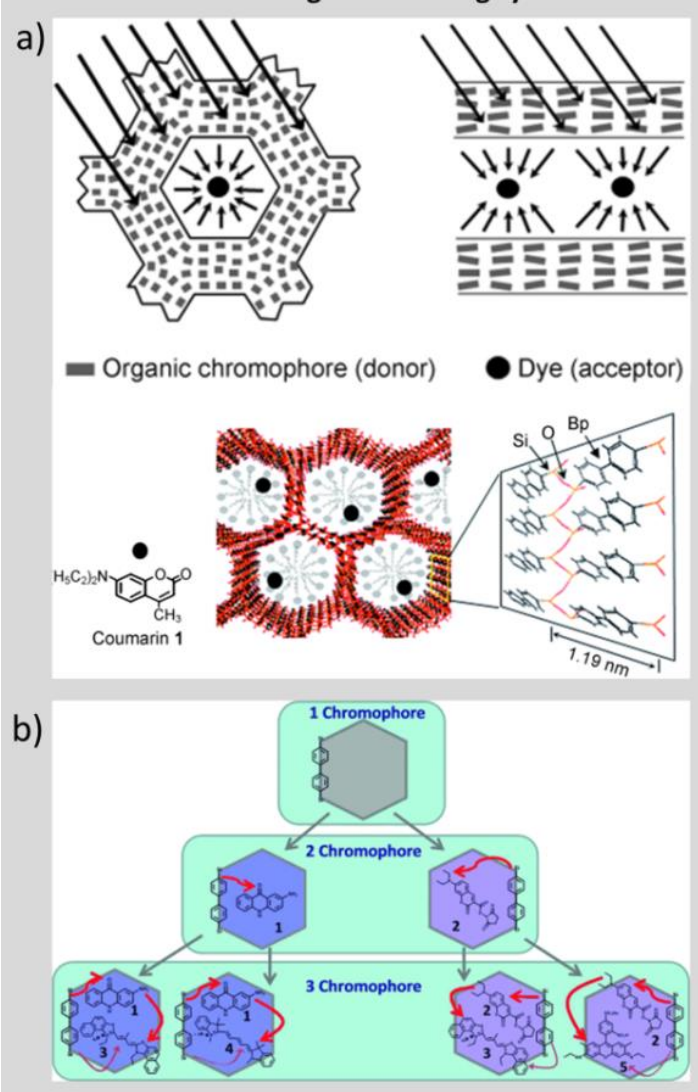

Figure 16. Embedding chromophores in the pore walls: Energy transfer from the chromophores incorporated in the pore walls of a PMO to the dyes inside the mesochannels. [Adapted with permission from Wiley- $\mathrm{VCH}$. ${ }^{[63]}$ b) The three-chromophore systems with light-harvesting behavior of Ref. ${ }^{[376]}$, for which a two-step-Förster resonance energy transfer process was detected. Energy is transferred from the biphenyl moieties embedded in the pore walls to mediators (indicated as (1)), and then to the acceptors (indicated as (3), (4), (5)) The emission wavelength can be varied from 477 to $630 \mathrm{~nm}$ by a proper selection of the acceptor dye. [Reprinted with permission from Wiley- $\mathrm{VCH}$.] $]^{[376]}$

Both silica and organosilica porous materials offer control over pore size and morphology. ${ }^{[350,377]}$ Yet the possibility to fine-tune the composition of the organosilica pore walls and the immense platform of functional precursors makes these hybrids very versatile materials, as nicely discussed in two reviews focused on their use in catalysis, sensing and optical applications. ${ }^{[353,378]}$ In this regard, the incorporation of chromophores in the organosilica walls, along with a proper selection of photoactive guest species is an appealing option, which has already produced materials for light-harvesting devices. ${ }^{[50,63,356,376]}$ For example, the Inagaki group devised an ingenious light-harvesting system by exploiting the biphenyl bridges in the PMO walls as the light absorbing donors, while the incorporated coumarin dye acted as acceptors (Figure 16a). By investigating the effect of the acceptor loading, it was found that the quantum yield of the material doubled by increasing the coumarin content by $0.8 \%$. The system showed a FRET efficiency, close to $100 \% .{ }^{[63]}$ The idea to use the organic bridges of biphenyl-PMO as donors proved to be successful also when two types of dyes were encapsulated (Figure 16b). Grösch et. al observed two-step-FRET from the biphenyl moieties to a mediator dye, followed by energy transfer from the mediator to the acceptor dye, with efficiencies ranging from 70 to $80 \%$. Interestingly, the wavelength of the emission could be tuned from 477 to $630 \mathrm{~nm}$ by a suitable choice of acceptor dye. ${ }^{[376]}$ 


\subsubsection{The inner surfaces of mesoporous silica materials}

The experimentally determined values of the pore thickness in mesoporous materials range between 7 and $15 \AA$. Pores surfaces may have different degrees of hydrophilicity depending on the surface density of the exposed silanol groups (normally from 1.5 to $7 \mathrm{SiOH} / \mathrm{nm}^{2}$ ) and are very difficult to characterize, due to their amorphous nature and high density of defects. ${ }^{[379]}$ Modeling greatly helps to characterize the surface of these materials. ${ }^{[379,380]}$ Models explicitly considering the quantum nature of chemical bonding would be highly desirable, yet this route implies higher computational costs. Earlier first principles studies ${ }^{[379]}$ utilized flat surface models, or nanometer-thick slabs, ${ }^{[381-383]}$ to simulate the walls of mesoporous materials providing insight into their defects. Combined with IR and UV-vis data, those calculations showed that strained surface defects such as two-membered rings are energetically disfavored over non-bridging oxygen defects. ${ }^{[381,382]}$ Extended models were then used to study high-temperature molecule-surface interactions, ${ }^{[384,385]}$ and to characterize Ti sites in Ti-grafted mesoporous silica jointly with IR/UV-vis spectra. ${ }^{[383]}$ Slit-pore models provide information on adsorption processes dominated by local features of the surface ${ }^{[379]}$ and are widely used to study defects, ${ }^{[386]}$ functionalization of pore surfaces, ${ }^{[387,388]}$ interaction with drugs, ${ }^{[389]}$ aminoacids, ${ }^{[390,391]}$ or peptides, ${ }^{[392]}$ and confinement effects on liquids, ${ }^{[393,394]}$ often unveiling intriguing facts. For example, molecular dynamics simulations showed that, in the presence of $\mathrm{Na}^{+}$and $\mathrm{K}^{+}$cations, a negatively charged peptide (pepsin) solvated by a water droplet can be attracted by a negatively charged silica surface. ${ }^{[392]}$ The peptide-silica interaction was rationalized thanks to a detailed study of the electrostatic potential of the peptide surface, ${ }^{[395]}$ which exhibited both negative and positive regions. ${ }^{[396]}$ Such model allowed to explain why encapsulation of pepsin in SBA-15 occurs at pH-conditions where both protein and mesoporous silica are negatively charged. ${ }^{[397]}$

Recently, computational studies with slit-pore models highlighted interesting organizational properties of confined fluids. ${ }^{[393,394,398,399]}$ According to Guo et al, an ethanol-water mixture undergoes a partial demixing within the walls of an hydrophilic pore: while ethanol molecules tend to adsorb on surface silanols, water molecules preferentially interact among themselves, forming wires or clusters (Figure 17a). ${ }^{[393]}$ By attaching to the hydrophilic walls, ethanol creates an hydrophobic coat, which could explain why water molecules prefer to stay away from the walls and to self-organize in clusters. ${ }^{[393]}$ Partial separation of ethanol and water was also found in model slit pores of graphene and boron nitride. Specifically, Kommu et al. observed a higher tendency of ethanol to selectively adsorb on the pore walls of boron nitride, and showed that the ethanol/water partial demixing was relatively unaffected by the pore width (Figure 17b). ${ }^{[394]}$ Similar microphaseseparated tubular structures seem to be a recurrent feature of binary liquid mixtures in mesopores, and are also supported by experimental evidences. ${ }^{[398-401]}$ For example, neutron diffraction experiments on MCM-41 filled with a tert-butanol-toluene mixture evidenced inhomogeneous distribution of the components, suggesting that the pore surface is covered by tert-butanol molecules, while toluene preferentially locates in the central region of the pore. ${ }^{[400]}$

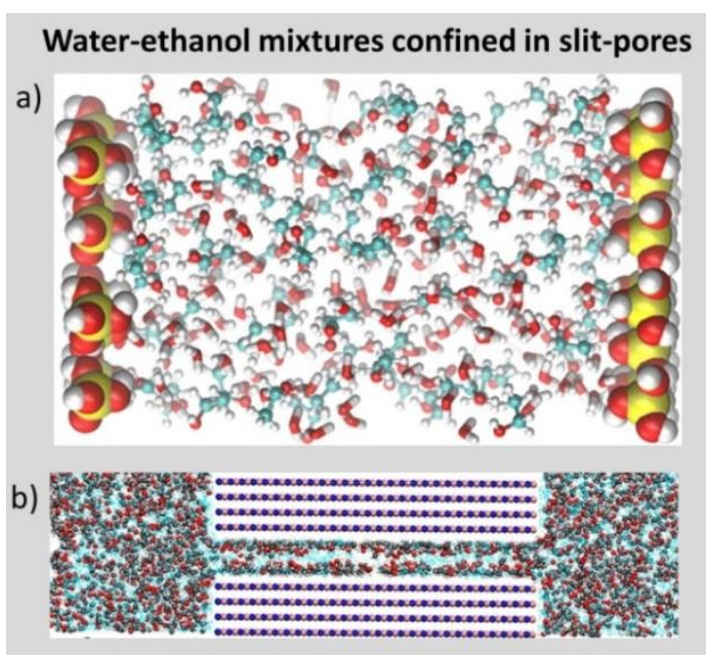

Figure 17. Organization of water-ethanol mixtures confined in slit pores of a) hydrophilic silica; b) boron nitride. Partial demixing occurs in both cases. a) Color codes: $\mathrm{Si}=$ yellow, $\mathrm{O}=$ red, $\mathrm{C}=$ cyan, $\mathrm{H}=$ white. [Adapted with permission from ref. ${ }^{[393]}$. Copyright 2014 American Chemical Society] b) Spheres represent ethanol atoms $(\mathrm{O}=$ red, $\mathrm{C}=$ gray $\mathrm{H}=$ white $)$. Cyan dots represent water molecules. Boron-nitride layers: $\mathrm{B}=$ pink, $\mathrm{N}=$ blue. Pore size: $13 \AA$. [Adapted with

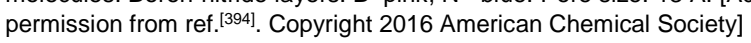

Theoretical models well illustrate the role of surface silanols in the arrangement of guest species. Ugliengo et al. ${ }^{[402]}$ developed a periodic hybrid-DFT model of the hexagonal MCM-41 pores, (Figure 18), with pore diameter $\sim 30 \AA$, walls $\sim 10 \AA$ thick, and silanol density $\sim 7 \mathrm{OH} \cdot \mathrm{nm}^{-2}$. Such model was used to study the absorption of water ${ }^{[403]}$ drugs, $^{[404]}$ and dye molecules ${ }^{[405]}$ in MCM41. Accurate treatment of hydrogen-bond interactions between surface $\mathrm{Si}-\mathrm{OH}$ groups and the adsorbates, ${ }^{[402]}$ and inclusion of dispersion ${ }^{[379]}$ allowed for a realistic picture of the encapsulation of the ibuprofen drug. ${ }^{[406]}$ As SS-NMR experiments indicated mobility of ibuprofen inside the mesopores, ${ }^{[152]}$ to foresee the most favorable absorption sites the investigators classified the inner surface in six regions with different silanol density and electrostatic potential. In particular, the $\mathrm{H}$ atoms of the silanols are associated with positive values of the potential (blue color in Figure 18b), while $O$ atoms with negative values (red color in Figure 18b). ${ }^{[406]}$ Such electrostatic maps indicated that the six regions are hydrophilic and can thus adsorb hydrophilic moieties like the ibuprofen carboxyl group. Accordingly, the ibuprofen absorbed on all six regions, but with different arrangements depending on the local environment of the site. The calculated structure, containing seven molecules per pore, shows a roughly uniform repartition of the drug on the pore surface, but without short-range ordering of the molecules (Figure 18c), because of the amorphous nature of the pore walls.

Experimental evidences has long supported the heterogeneity of the MCM-41 pore surface, which may be described as composed by hydrophilic (silanol-rich) and hydrophobic (silanol-poor) patches. ${ }^{[407,408]}$ A similar picture (Figure 19a) was obtained by simulating by molecular dynamics the dehydration process of a cylindrical pore via condensation of adjacent silanols. ${ }^{[09]}$ Despite the initially uniform silanol distribution, after dehydration the silica pore displayed a slightly elliptical shape, with patches of hydrophobic or hydrophilic character, respectively located in highand low-curvature regions of the pore surface. 
a)
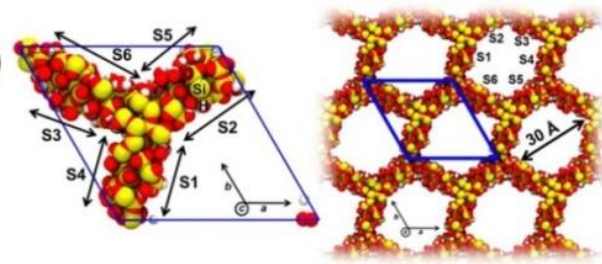

b)

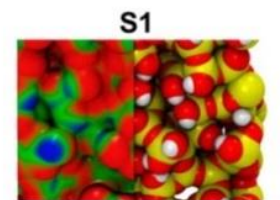

S3

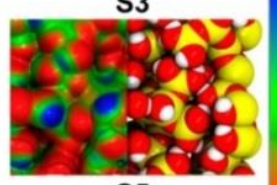

S5
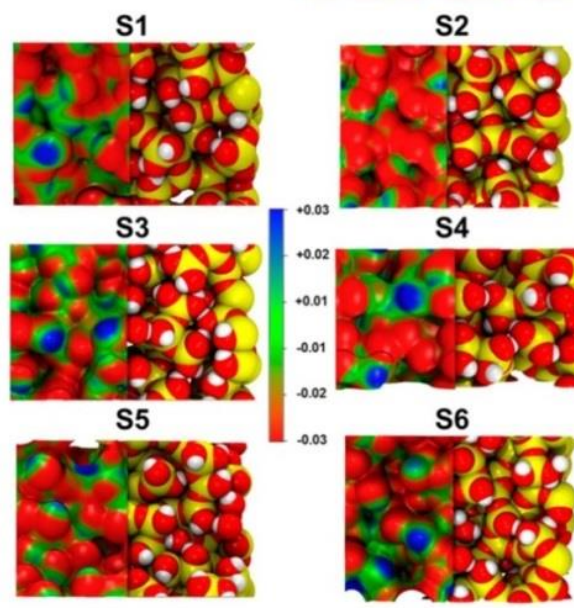

S4

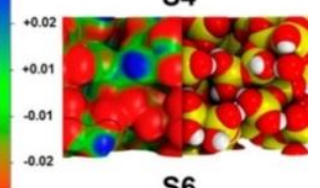

S6

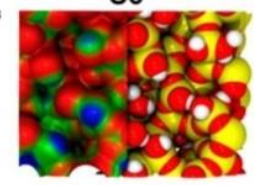

c)

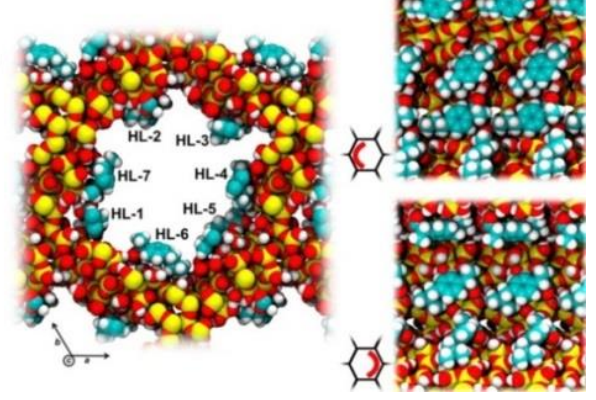

Figure 18. MCM-41 "in-silico": Unit cell (blue lines) and packing of the MCM41 model adopted in Ref. ${ }^{[406]}$ to study the adsorption of ibuprofen. Both views are along the $c$ axis. The six internal regions of the pore are identified as S1-S6. b) The right panels show the structure on top of which (left strip) the electrostatic potential was mapped on the electron density isosurface (used isovalue: $0.0004 \mathrm{e}$ ). The color scale indicates the electrostatic potential values c) MCM-41 with 7 ibuprofen molecules adsorbed in the pore. (Left) View along $c$ axis. (Right) Lateral view showing the two halves of the pore. Color codes: $\mathrm{Si}=$ yellow, $\mathrm{O}=$ red, $\mathrm{C}=$ cyan, $\mathrm{H}=$ white. Adapted with permission from Ref. ${ }^{[406]}$ Copyright 2014 American Chemical Society.

According to these simulations, the silanol condensation process could be at the origin of the non-uniform $\mathrm{Si}-\mathrm{OH}$ distribution on the surface. Similar deformation was also found in the first-principles simulation of a silica pore with a larger diameter, initially exhibiting a perfect cylindrical shape (Figure 19b). ${ }^{[410]}$

Various procedures allow to build pore models for computer simulations. ${ }^{[411]}$ Nevertheless, the pore surface still remains very difficult to capture at atomistic detail. Whereas the pores of MCM-41 are non-connected, SBA-15 materials are more complex: they have intra-wall disordered micro-porosities, ${ }^{[412]}$ or small transversal channels connecting the pores, as indicated by the evolution of ${ }^{129} \mathrm{Xe}$ NMR chemical shift with $\mathrm{Xe}$ partial pressure. ${ }^{[150]}$ These defects are likely due to the penetration of the template chains in the SBA-15 silica framework. ${ }^{[413-415]}$

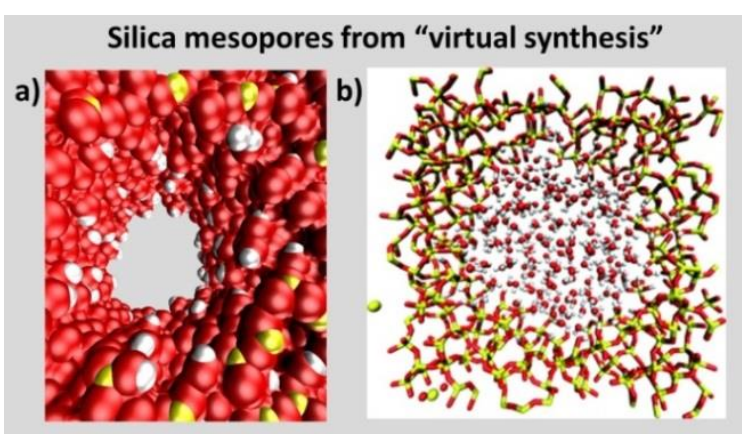

Figure 19. Silica mesopores obtained by virtual syntheses using a) forcefield ${ }^{[409]}$ and b) first principles ${ }^{[410]}$ simulations. In both cases, the pore shows an elliptical shape, and silanols are more densely distributed in the flatter regions of the pore surface. Color codes: $\mathrm{Si}=$ yellow, $\mathrm{O}=$ red, $\mathrm{H}=$ white. In b), the pore also contains water molecules. [Reprinted with permission from Wiley- $\mathrm{VCH}$.] ${ }^{[410]}$

The connectivity among mesochannels has been visualized by single-molecule fluorescence microscopy (see Ref. ${ }^{[416]}$ for a review). In these experiments, luminescent probe molecules were incorporated in as-synthesized mesoporous silica, and their diffusion along the channels was followed with a fluorescence microscope. ${ }^{[417]}$ The passage of dye molecules between channels was detected, thus proving that the pores were connected. ${ }^{[418]}$ Experiments with an orienting electric field and molecules of different size shed light on the local surface features, identifying leaky defects in the walls of the pores. ${ }^{[419]}$

To summarize, mesoporous materials incorporate guests that would never fit into zeolites: they have tunable pores and are relatively easy to functionalize. However, the amorphous structure of the walls implies that the surface functionalities (and hence the guest molecules) do not have a regular distribution at the molecular scale, and that the behavior of the included molecules is actually dominated by the surface defects. Also, small-sized guests still preserve much freedom of motion, which hinders the formation of uniform and stable supramolecular arrays.

\subsection{Metal-organic and covalent porous frameworks}

Metal organic frameworks (MOF) are typically constituted by inorganic secondary building units, often containing a transition metal center, and organic linkers, joined through moderately strong coordination bonds. ${ }^{[420]}$

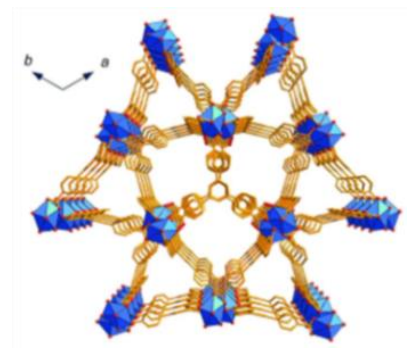

Figure 20. A MOF. The inorganic building units (blue polyhedra) are connected by the organic linkers. The MOF is viewed along the $c$ direction (color codes: In=blue polyhedra; $\mathrm{C}=$ orange; $\mathrm{O}=$ red). By ion exchange, dyes can be orderly encapsulated in this MOF, leading to second-order nonlinear optical (NLO) materials ${ }^{[62]}[\text { Reprinted with permission from Wiley-VCH. }]^{[62]}$ 
One of them is shown in Figure 20: the peculiar triangular shape of its 1D channels, very different from elliptical / circular openings of zeolites or silica mesopores, suggests new opportunities for space confinement. ${ }^{101]}$ Indeed, this MOF was shown to incorporate dipolar dyes by simple ionic exchange, yielding a composite with high second-harmonic generation intensity. ${ }^{[62]}$

Covalent organic frameworks, (COF) - which are made only by organic building blocks, and can be considered as the lightweight counterparts of mesoporous silicas or zeolites. They are useful in applications where low weight and high framework flexibility are required, with the additional benefit that advanced functionalities, such as chirality or photoconduction, ${ }^{[421]}$ can be directly incorporated into the framework by properly selecting the ligands and the synthesis conditions. ${ }^{[422]}$ Two-dimensional COFs have a layered structure deriving from $\pi$-stacking of planar "building units" with extended $\pi$-conjugated systems amenable for electronic and optoelectronic applications. ${ }^{\left[{ }^{[1,421]}\right.} \mathrm{A}$ nice example is the COF in Figure 21: the stacking of the conjugated units creates a tubular network within which bulky guests can be confined. This was the case of $\mathrm{C60}$, which was incorporated into the COF via sublimation. Because of host-guest size restrictions, only one $\mathrm{C}_{60}$ molecule can pass through the cylindrical pores. Upon irradiation with visible light, the composite displayed photoconductivity and fast response to the light stimulus, making this material particularly interesting for photoswitches and photovoltaic cells. ${ }^{[421]}$
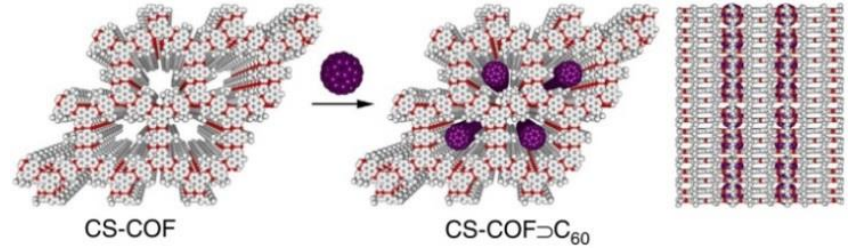

Figure 21. A $\boldsymbol{\pi}$-conjugated $\mathbf{C O F}$ in van-der-Waals representation (grey carbon, red: nitrogen, tert-butyl groups and $\mathrm{H}$ atoms not shown). The material is built from the stacking of $\pi$-conjugated layers, thus extending the periodic structural ordering of the layers to the third dimension. It is an ideal host for bulky guests: upon $\mathrm{C}_{60}$ inclusion, the composite displayed photoconductivity. ${ }^{[421]}$ [Adapted by permission from Macmillan Publishers Ltd. ${ }^{[421]}$ ]

Zeolite Imidazolate Frameworks (ZIF) have high robustness and chemical stability, ${ }^{[423,424]}$ while pore dimensions are smaller compared to other MOF families. Although topologically isomorphic with zeolites, ZIF are made up by imidazolate linkers connected to transition metal centers in tetrahedral coordination. Their zeolite-like topologies derive from the fact that the metalimidazolate-metal angles are close to the $\mathrm{Si}-\mathrm{O}-\mathrm{Si}$ inter-tetrahedra angles. Figure 22 shows a popular member - ZIF-8, an archetypal example of MOF's resistance to pressure. ${ }^{[423]}$ ZIF-8 is also a choice system for modeling studies, aimed at the molecular-level description of the geometry ${ }^{[424]}$ and flexibility ${ }^{[425]}$ of the pore windows, useful in sorption applications, or the modification of its band structure for electronic or photocatalytic uses. ${ }^{[426,427]}$

Zeolite-like frameworks can be built with other linkers besides imidazolates, and are usually termed ZMOF (Zeolitic MOF). For instance, the ZMOF in Figure 23 has SOD topology and was obtained using organic building units designed to be the tetrahedral nodes in the final structure, and $\ln ^{3+}$ ions. ${ }^{[428]}$

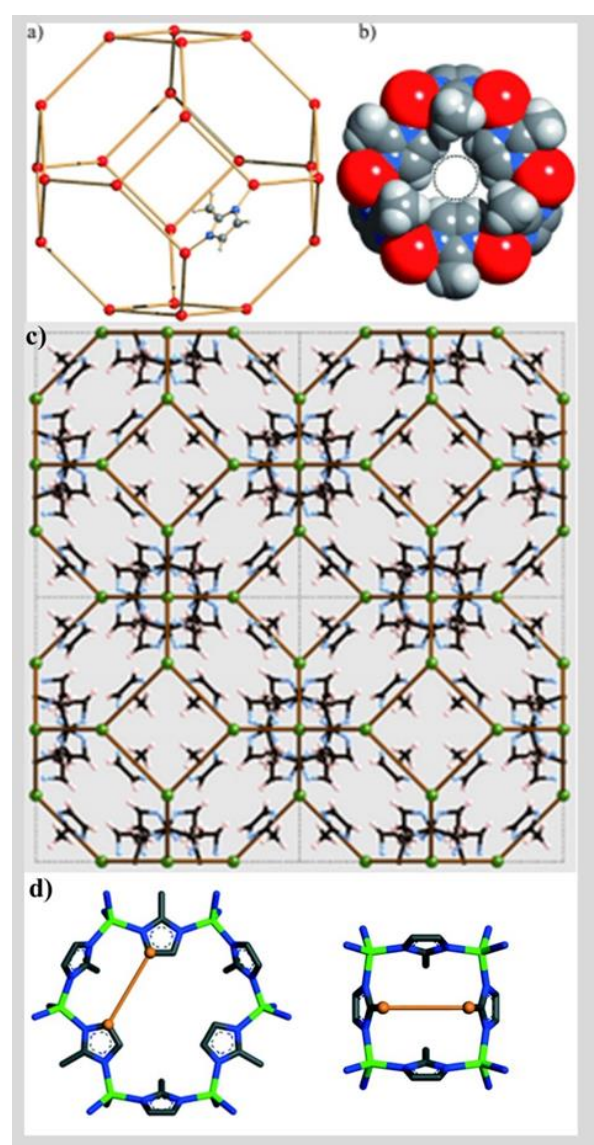

Figure 22. $\boldsymbol{A}$ ZIF with sodalite topology. ZIF- $8^{[423]}$ has composition $\mathrm{Zn}(\mathrm{mim})_{2}$ (where $\mathrm{mim}=2$-methylimidazolate), and sodalite (SOD) topology. a) A topologic SOD cage, showing the typical six-ring (hexagonal) and four-ring (rhombic) windows. A mim ligand connecting two $\mathrm{Zn}$ centers (red balls) is also highlighted. b) Space-filling representation of a six-ring window of the SOD cage with $3.3 \AA$ diameter. Color codes: $\mathrm{Zn}=$ red, $\mathrm{N}=$ blue, $\mathrm{C}=$ gray, $\mathrm{H}=$ white. [Adapted with permission from Wiley- $\mathrm{VCH}$. $]^{[423]}$ c) Ball-and-stick representation of a $2 \times 2$ supercell of ZIF-8. The SOD topology is highlighted by brown lines connecting $\mathrm{Zn}^{2+}$ ions (green balls). [Adapted with permission from Wiley-VCH.] ${ }^{[425]} \mathrm{d}$ ) Balland-stick representation of the six-membered and four-membered rings in ZIF-8. Colors: $\mathrm{Zn}=$ green, $\mathrm{N}=$ blue, $\mathrm{C}=$ black ( $\mathrm{H}$ not shown). The orange segments indicate the $\mathrm{C}$-C which define the width of the window $(4.946 \AA$ and $4.608 \AA$ for six- and four-membered rings, respectively). ${ }^{[424]}$ [Reproduced from Ref. ${ }^{[424]}$ with permission from The Royal Society of Chemistry]

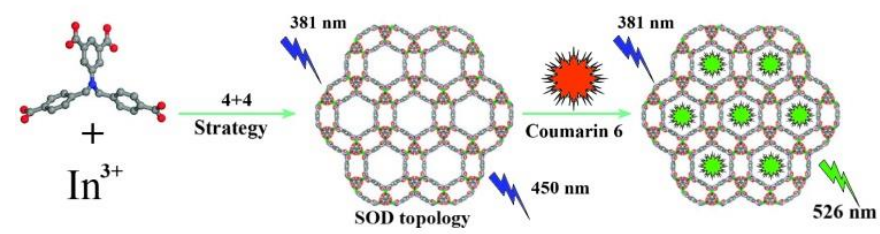

Figure 23. A ZMOF with sodalite topology. This ZMOF, constituted by $\ln ^{3+}$ and a tetracarboxylate ligand, has a negatively charged framework with SOD topology and emits in the visible. By encapsulation of cationic dyes such as coumarin, it shows interesting light-harvesting properties. [Reproduced from Ref. ${ }^{[64]}$ with permission from The Royal Society of Chemistry]

Such a ZMOF was loaded with coumarin dye by ionic exchange. Photoluminescence spectra of the composite evidenced that the excitation energy was transferred from the ZMOF host to the guest dyes, indicating an efficient light-harvesting behavior.

A wide variety of MOFs frameworks has been reported, featuring systems of 1D- , 2D-, or 3D- channels similar to zeolites, and/or isolated cages analogous to clathrates. Cavities of various shape 
have been designed, and functionality has been imparted to the framework using catalytically active metal centers. ${ }^{[429,430]}$ Also the linkers often contain active, stimuli-responsive elements, such as rotors, ${ }^{[431]}$ rotaxanes $^{[432]}$ and catenanes, ${ }^{[433,434]}$ or luminescent species. ${ }^{[435]}$ Several MOFs incorporate functional elements both within their framework and inside the pores. Figure 24, for example, illustrates the possibility to tune the rotor motion of the linkers by $\mathrm{CO}_{2}$ loading/unloading in the MOF, as demonstrated by ${ }^{2} \mathrm{H}$ SS-NMR spectroscopy. ${ }^{[431]}$
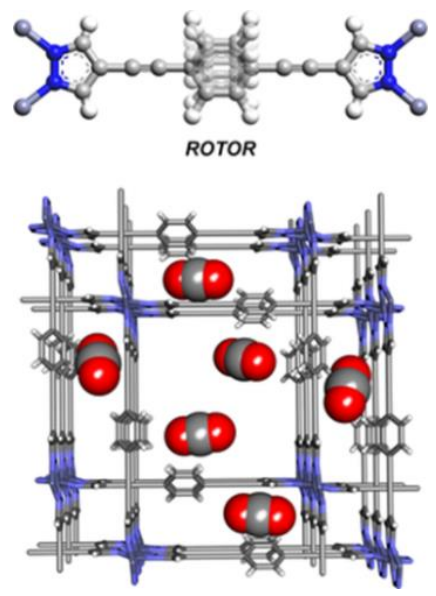

Figure 24. A stimuli-responsive MOF framework, where the rigid rod-like linkers bear a phenylene rotor (top). The spinning speed of the rotors is tuned by the $\mathrm{CO}_{2}$ entering the MOF. [Reprinted with permission from Wiley-VCH.] ${ }^{[431]}$

Intense growth of this area (for an overview see e.g., CrystEngComm, 2015, issue 2; Coord. Chem. Rev. 2016 - Issue 307; Chem Soc Rev 2017, Issue 11), has led to the formulation of general rules for classifying MOF's structures, which can be retrieved in dedicated databases. ${ }^{[436]}$

Such functional and stimuli-responsive platforms for the confinement of molecules or nano-objects have opened new perspectives not only to devices (see e.g. Ref. ${ }^{[30]}$ for a review) but also to analytic methodologies. The so-called "crystalline sponge" approach refers to the preparation of composites in which guest molecules are trapped into MOF cages and then studied by crystallographic methods. ${ }^{[268,437]}$ In practice, carefully selected frameworks absorb target molecules from a solution, thus converting the incoming liquid into an arrangement of confined guest species thanks to the molecular-recognition ability of MOF cavities. ${ }^{[438]}$ In this manner, the geometrical features of the absorbed species could be revealed, along with the host framework, by X-ray analysis of the porous composite material, thus making structure elucidation possible even for compounds very difficult to crystallize. ${ }^{[437,438]}$ Besides the obvious advantages, the method suffers from limitations: i) structure resolution is not always successful, ii) pore confinement in some cases alters significantly the physicochemical properties of both the guest species and the host, as evidenced by a recent study. ${ }^{[439]}$

\section{Clusters of metals or semiconductors: organization of zero-dimensional nanosystems}

Zeolites have been elected as ideal matrices for creating ordered arrays of clusters of metal or semiconductors since the early 80 's. ${ }^{[14]}$ Semiconductor nanoclusters, widely known as quantum dots, are an ever-green topic in material science: they offer the unique opportunity of synergically combining quantum size effects (useful for optoelectronic applications) with an exceptionally high fraction of exposed surface atoms, that would be desirable for catalysis. Unfortunately, the latter feature is usually accompanied by a too high reactivity of the clusters, and by the unwanted formation of larger aggregates. Confinement in porous matrices was early recognized as a viable solution for those problems; hence zeolite frameworks - chemically inert and characterized by remarkable thermal and mechanical stability - were immediately exploited to this aim. Ideally, both the high reactivity and the growth of the clusters would be limited by the small and regular size of zeolite pores. Practically, the implementation of this concept encountered several difficulties, which hindered the fabrication of regular, ordered architectures of clusters in zeolite cavities until very recent times. Although many approaches to the production of uniform, low-dimensionality nanostructures have been implemented over the years, ${ }^{[187,440]}$ fabrication in confined spaces never lost its appeal. In particular, the improvement of synthesis and characterization techniques ${ }^{[41-445]}$ combined with insight from computational studies ${ }^{[446-448]}$ fostered this decade's tremendous progress. ${ }^{[43,449]}$

Common synthetic strategies for confined nanoclusters are vapor deposition of suitable precursors, ${ }^{[14,16]}$ or "ship-in-a-bottle"[450,451] approaches allowing for the in-situ synthesis of compounds in a host matrix. In the latter case, the reactants are molecules and ions sufficiently small to penetrate inside the host nanochannels, leading to products too large to escape from the cavities.

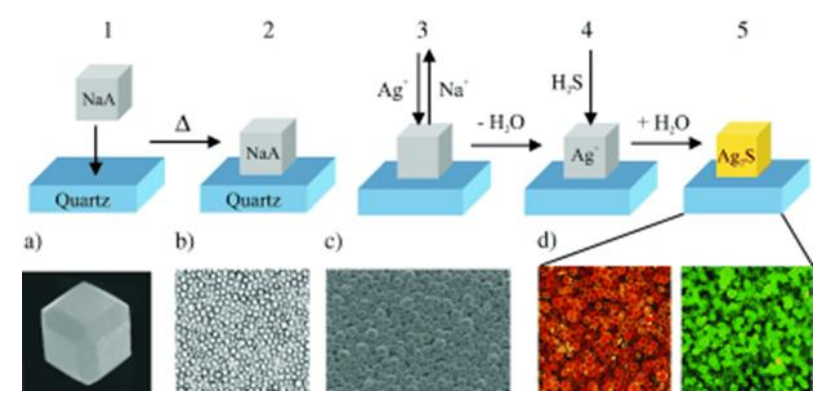

Figure 25. Ship-in-a-bottle" synthesis of confined nanoclusters: 1) physisorption of monolayer of zeolite A crystals on quartz; 2) fixation of the monolayer by heating $\left(600^{\circ} \mathrm{C}\right)$; 3) $\mathrm{Ag}^{+}$insertion via ion-exchange; 4) dehydration and exposure to $\mathrm{H}_{2} \mathrm{~S}$ vapor, which reacts with enclosed $\left.\mathrm{Ag}^{+} ; 5\right)$ rehydration and formation of stable $\left(\mathrm{Ag}_{2} \mathrm{~S}\right) n$ clusters in zeolite $\mathrm{A}$. The images show the zeolite crystals during the process. [Reprinted with permission from Wiley- $\mathrm{VCH}$.] ${ }^{[39]}$ 


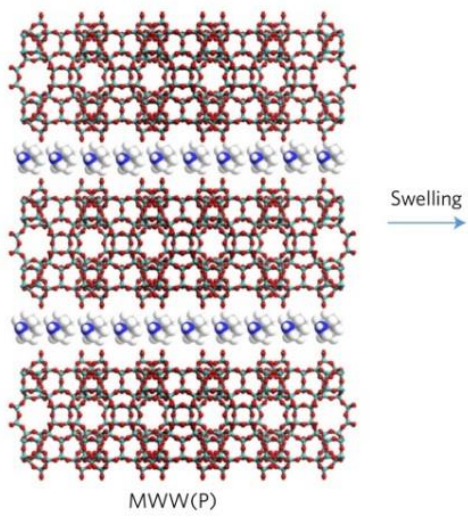

Hexamethylenimine
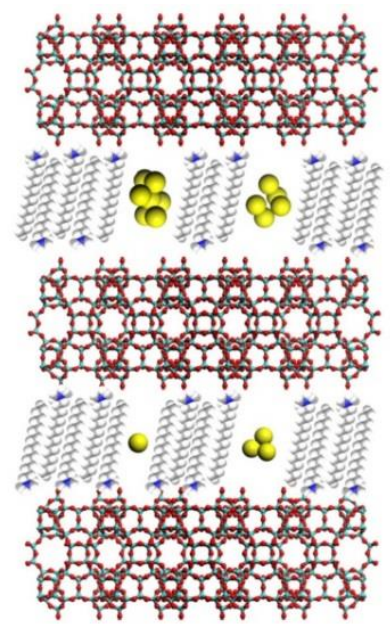

,

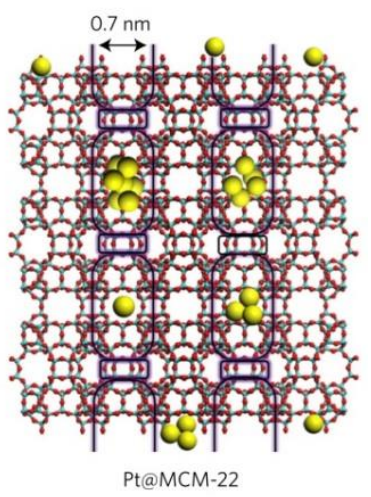

Pt cluster

Figure 26. Fabrication of Pt-nanocatalysts confined in zeolite MCM-22 (MWW-framework type). ${ }^{[58,60]}$ A Pt-containing solution is added during the swelling process of a layered zeolitic precursors. The $2 \mathrm{D}$-zeolite sheets are expanded using a common organic surfactant (hexadecyltrimethylammonium, $\mathrm{CTMA}^{+} \mathrm{OH}^{-}$). Smaller Pt species are also encapsulated inside the channels of each zeolitic layer. Removal of the template leads to the Pt@MCM-22 composite material, featuring supercages of $\sim 0.7 \times 1.8 \mathrm{~nm}$ size, loaded with Pt single atoms or subnanometric Pt clusters. Reprinted by permission from Macmillan Publishers Ltd. ${ }^{[123]}$

This concept is illustrated in Figure 25 for the "ship-in-a-bottle" fabrication of luminescent silver sulfide clusters in supported zeolite A monolayers. ${ }^{[39]}$ Silver ions enter zeolite cages via ionic exchange, and self-assemble into clusters upon heating or irradiation with light in a process usually called "activation".

A new strategy for the fabrication of zeolite-confined nanocatalysts was recently proposed by the Corma group, based on the incorporation of $\mathrm{Pt}$ during the transformation of a twodimensional into three-dimensional zeolite. ${ }^{[452]}$ The idea was to entrap Pt clusters inside the hemi-cages of a layered zeolite precursor during the condensation step (see Figure 26). Besides outstanding thermal stability and catalytic activity, the obtained material featured a distribution of confined Pt-species, comprising both individual atoms and nanoclusters up to $1 \mathrm{~nm}$ in size. This example clearly showcases how difficult it is to synthesize arrays of confined clusters with atomic-level order. Irrespective of type of nanostructures and fabrication strategy, perfectly ordered arrays of e.g. nanodots might be obtained only if the pores were uniformly occupied - thus, in practice, only if the confining matrix were completely filled by species of the same size. This objective is very hard to achieve even by using zeolites with a single type of pores of small size, such as sodalite - which is constituted only by small-sized cavities called beta-cages (see Figure 26).

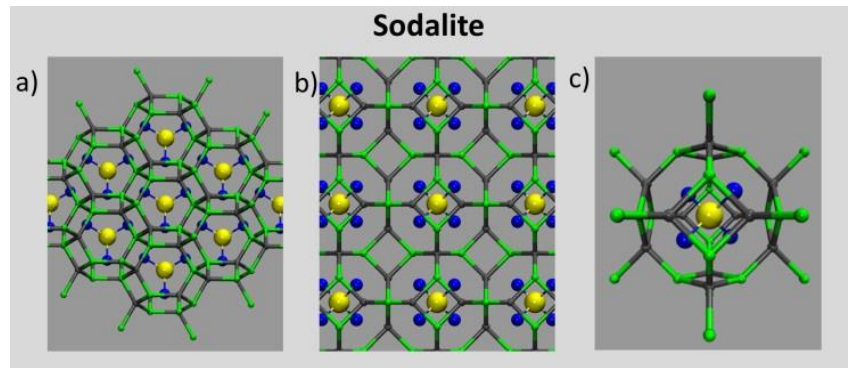

Figure 27. Natural sodalite (SOD framework type). a,b) Two representations highlighting: a) the six-membered-ring windows of the cavity; b) the cubic symmetry of the framework, derived by the infinite replication of a sodalite-cage (shown in c)). Color codes: $\mathrm{Si}=$ grey; $\mathrm{Al}=$ green; $\mathrm{Cl}=$ yellow; $\mathrm{Na}=$ blue (the

framework oxygen atoms are not shown for clarity). [Illustration created with VMD, using the X-ray positions from Refs $\left.{ }^{[58,60]}\right]$

Syntheses inside mesoporous matrices were also plagued by these problems for a long time: although nanostructures of metals or metal oxides have been fabricated since end 1990's, ${ }^{[453]}$ in most cases, the pores were partially filled, and the resulting distribution of clusters or nanorods in the mesoporous-silica matrix was quite random. ${ }^{[359]}$ Nonetheless, perfect order is not required for most applications, including catalysis or sensing.

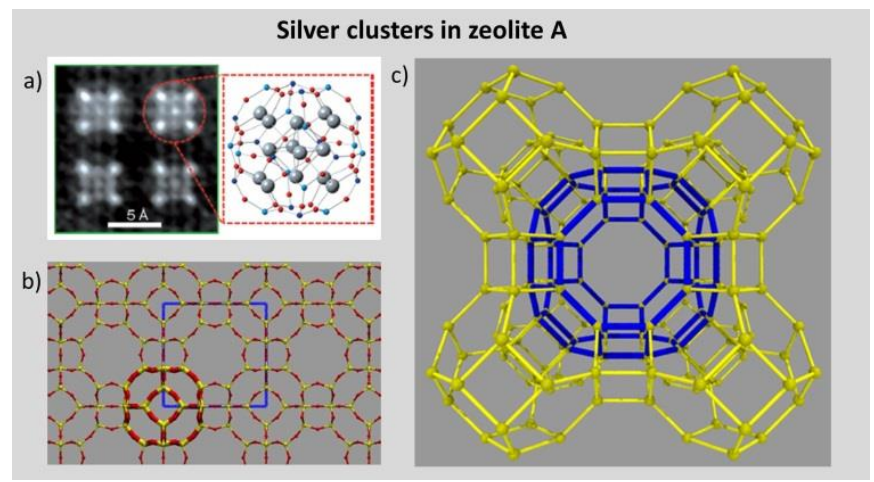

Figure 28. The void-space architecture of zeolite $\boldsymbol{A}$ features cages of different size, among which silver cluster of outstanding luminescence can be hosted. a) Scanning transmission electron microscopy image of the arrangement of isolated $\mathrm{Ag}$ ions and $\mathrm{Ag}$ clusters of six atoms in zeolite $\mathrm{A}$. The sodalite cage is shown in the left inset. [Reprinted by permission from WILEY$\mathrm{VCH}^{[441]}$ b) Framework structure (yellow $=\mathrm{Si}$, red $=0$ ) of zeolite $\mathrm{A}(\mathrm{LTA})^{[58,60]}$ with the unit cell shown as solid blue lines. The sodalite cage, shown in licorice representation, is a composite building block of zeolite A. c) Representation of the LTA framework highlighting the sodalite cages (yellow) and the alpha-cage (blue) [Illustration created with VMD, using the X-ray positions from Refs ${ }^{[58,60]}$.]

\subsection{Nanoclusters in sodalite cages}


No surprise at all that the pioneering efforts to synthesize nanoclusters in zeolites chose sodalite as their favorite playground (Figure 27) ${ }^{[14]}$ Its beautifully symmetric body-centered arrangement of perfectly equal cavities - the truncated-octahedral beta cages - also captured the attention of Linus Pauling, who first described its structure in 1930. ${ }^{[454]}$ The sodalite structure builds up the most numerous zeolite family, counting about 900 members, i.e. over $18 \%$ of all published zeolite structures, ${ }^{[455]}$ among which lazurite ${ }^{[66,456]}$ - the main component of lapis-lazuli, and natural tsaregorodsevite - a rare mineral encaging tetramethylammonium cations. ${ }^{[320,457]}$ The SOD beta-cage is also a building block of other zeotypes, like the catalytic cornerstone zeolite A (Figure 28), or zeolite Y (Figure 29), and a widespread structural motif in metal-organic framework materials. ${ }^{[64,458]}$

Also the first computational studies aimed at gathering insight on the structural and electronic properties of confined nanoclusters were performed on sodalite, ${ }^{[459-461]}$ which still remains a very attractive model system for theoretical investigations. For example, theory indicates that the electronic properties of isolated quantum dots are mainly transferred to the confined species, and that the zeolite environment just modulates their electronic transitions. ${ }^{[462,463]}$ Hence, frameworks act as dielectric media, that protect the clusters and maintain their optical properties. ${ }^{[463,464]}$

Since 1990's ${ }^{[41,42,465]}$ A and $Y$ zeolites have been used to stabilize silver cluster, and the appealing optical properties of the resulting composites have been explored. The observation that, upon high vacuum activation, the originally white-colored silver-exchanged zeolite A turned yellow at room temperature and brick red at $200{ }^{\circ} \mathrm{C}$ was particularly important because it revealed that the optical properties of the composite depended on hydration. ${ }^{[42]}$ The reversible, stimuli-induced change of colors of silver zeolites was correlated to ligand-to-metal charge transfer transitions from the oxygen atoms of the framework to the vacant $5 \mathrm{~s}$ orbital of the $\mathrm{Ag}$ ions. ${ }^{[42]}$ In later experiments, individual Ag clusters were activated by a laser beam, producing intense luminescence. ${ }^{[442]}$ Other studies showed that the emission of Li-substituted silver-loaded zeolite A could be tuned by water: while partially hydrated composites gave a blue emission, a green/yellow color was detected upon full hydration. ${ }^{[466]} \mathrm{XRD}$ and scanning transmission electron microscopy on silver-zeolite $A$ samples revealed that silver forms octahedral clusters inside the sodalite cages, surrounded by eight cations, each located in the center of a 6 membered-ring (Figure 28a). ${ }^{[441]}$ However, the size of the confined clusters depends, in general, on the loading and preparation history of the sample. Operating at relatively mild conditions, cationic aggregates composed by 3, 4, 6 silver atoms - namely, $\mathrm{Ag}_{3}{ }^{{ }^{+}}, \mathrm{Ag}_{4}{ }^{{ }^{+}}, \mathrm{Ag}_{6}{ }^{+}$were obtained; such clusters were characterized by EPR spectroscopy, testing also their reactivity towards ethylene, dioxygen and nitrogen oxide. ${ }^{[467-470]}$

Nowadays, silver-containing zeolites can be synthesized with tailored optical properties: ${ }^{[43]}$ they can emit in the whole visible spectrum $^{[471]}$ and with remarkable quantum efficiencies. ${ }^{[472]}$ Zeolite-Y works particularly well, because the luminescent clusters are hosted in well-separated hexagonal prism cages. ${ }^{[43,473]}$ In zeolite $A$, the clusters are located in adjacent sodalite cages (Figure 28), yet they still feature excellent stability and efficiency. ${ }^{[473]}$ By this virtue, silver exchanged LTA zeolites hold great promise for practical applications such as optical encoders ${ }^{[444]}$ or bright emitters in OLEDs. ${ }^{[45]}$ Further progress in this field could be triggered by deeper insight on the structural and electronic properties of the confined aggregates via firstprinciples studies. ${ }^{[447,474]}$ Also, since silver cations located at different sites have different enthalpies of adsorption, ${ }^{[475,476]}$ the use of thermodynamic models capable to predict the repartition of
$\mathrm{Ag}^{+}$cations in the confining medium upon ion exchange, and hence the organization of the Ag-clusters in the final composite, could advance the preparation procedures of these materials via a knowledge-based approach. ${ }^{[475,477]}$ Metals such as $\mathrm{Cu}^{[478]}$ or $\mathrm{Pt},{ }^{[479-481]}$ have also been incorporated in zeolite frameworks - for instance, nearly spherical $\mathrm{Pt}_{13}$ clusters showing interesting magnetic properties were realized inside zeolite $Y$ supercages, ${ }^{[479]}$ and confined nanoalloys can be produced as well. ${ }^{[482]}$

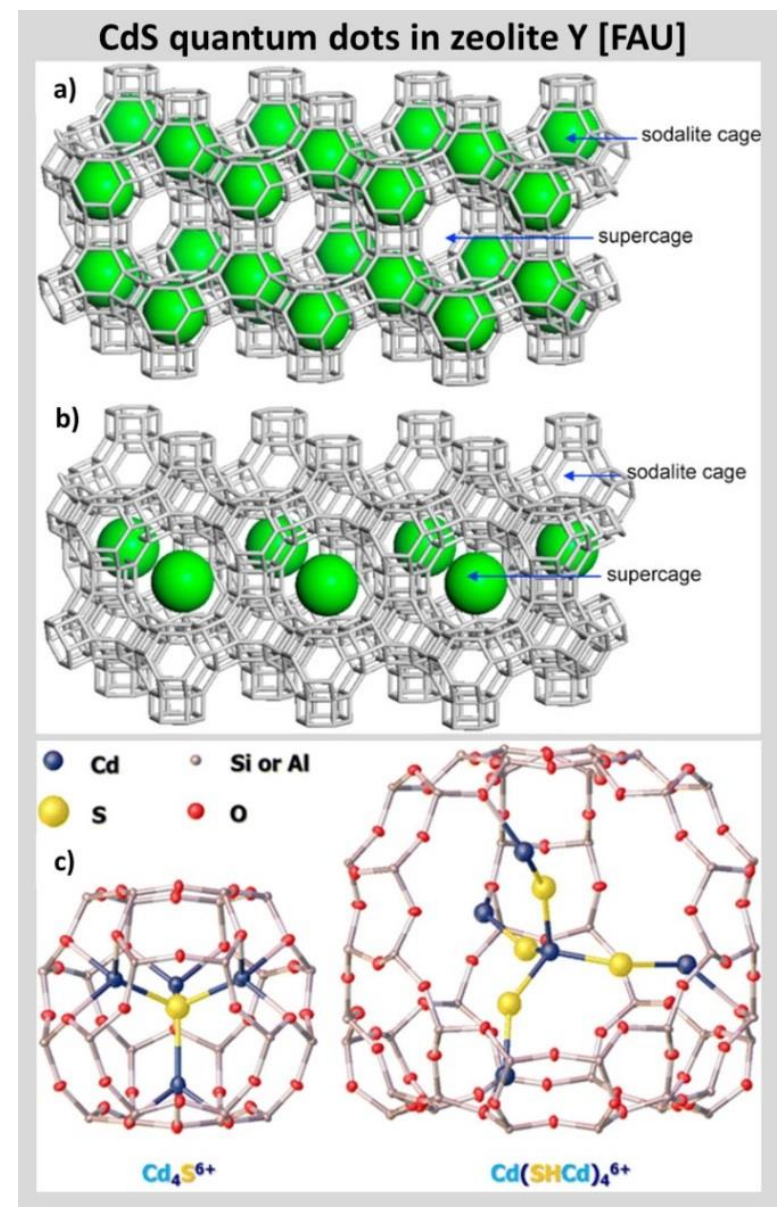

Figure 29. CdS quantum dots (schematically represented as green spheres) confined in zeolite $\mathrm{Y}$, located a) in both sodalite cages and supercages; b) in the supercages. [Reprinted by permission from Elsevier ${ }^{[37]}$ c) X-rays structure of tetrahedral $\mathrm{Cd}_{4} \mathrm{~S}^{6+}$ ion in the sodalite cage (left) and tetrahedral $\mathrm{Cd}(\mathrm{SHCd})_{4}{ }^{6+}$ ion in the supercage (right) of zeolite $\mathrm{Y}$. [Adapted with permission from Ref. ${ }^{[483]}$ Copyright 2016 American Chemical Society.]

Chalcogenide clusters have been widely studied, in particular using zeolite $Y$ as a matrix. ${ }^{[449,484,485]}$ As observed for the Agclusters, the structure of the confined aggregates and the properties of the resulting materials are strongly dependent on the composition of the framework and on process parameters, such as the degree of hydration. ${ }^{[485]}$ In principle, the clusters can form both in the sodalite cage and in the supercage of zeolite $Y$ (Figure 29a,b). Recently, a structural model based on X-ray diffraction (Figure 29c) has been proposed, suggesting that a tetrahedral ion $\left(\mathrm{Cd}_{4} \mathrm{~S}^{6+}\right)$ occupies the SOD cage (similar to natural sodalite, Figure 27), while a larger tetrahedral guest, $\mathrm{Cd}(\mathrm{SHCd})_{4}{ }^{6+}$ is located in the supercage. ${ }^{[483]} \mathrm{A}$ recent review thoroughly discusses the synthetic strategies to achieve, for example, the selective occupation of only one type of cages, and the broad spectrum of potential applications of these composite materials. ${ }^{[37]}$ For instance, photovoltaic solar cells based on CdS 
and $\mathrm{PdS}$ quantum dots encapsulated in zeolite $\mathrm{Y}$ have been realized, and the performance of such devices was found to depend, besides from the chemical nature of the nanospecies, on the homogeneity of their distribution inside the zeolite matrix. ${ }^{[486]}$

Chalcogenide nanoclusters have been also encapsulated into a chalcogenide-based semiconductor zeolite. ${ }^{[487]}$ Specifically, the host-guest hybrid system contained MnS clusters, as confirmed by UV-Vis, IR and Raman measurements. The aggregation state of the cluster, probed by EPR, suggested different morphologies according to degree of loading and temperature. Interestingly, the MnS clusters showed two emissions peaks (at 518 and $746 \mathrm{~nm}$ ) whose photoluminescence intensity depended on loading level and temperature. ${ }^{[487]}$ Indeed, the second peak was never found in previous experiments on MnS clusters in other hosts, raising thus interest toward the potential use of semiconductor zeolites as hosts for luminescent nanoclusters.

\subsection{Nanoclusters and quantum wires in mesopores}

Owing to their large pore sizes, mesoporous silicas are appealing hosts for quantum nanostructures, also because silica is an insulator (like most zeolites), and acts as an effective barrier between individual nanowires. ${ }^{[488]}$ Obtaining mesoscopic ordering of the confined nanomaterials is, in general, more difficult than in zeolites, because low crystallinity, or presence of unwanted macropores may lead to the growth of nanoparticles greater than the nominal pore size and to their sparse incorporation within the matrix. ${ }^{[453]}$ Despite these problems, advances in the fabrication procedures made it possible to realize, for example, arrays of highly ordered zinc-manganese sulphide quantum wires within MCM-41 and SBA-15, ${ }^{[488]}$ or magnetic $\mathrm{Fe}_{2} \mathrm{O}_{3}$ nanoparticles with regular, finely controllable sizes confined into hexagonally ordered mesopores (Figure 30a). ${ }^{[489]}$

Recently, intriguing alternatives to standard quantum dots, such as nanosized perovskites, have become very popular due to their excellent optical properties and photovoltaic performances. ${ }^{[490]}$ Whereas the photophysical properties of conventional quantum dots (chalcogenide nanoclusters) are very sensitive to surface defects, in such materials the surface dangling bonds do not create trap states that detrimentally affect photoluminescence. ${ }^{[491]}$ Because of this defect-tolerance, perovskite nanocrystals do not need to be passivated. The Kovalenko group took advantage of this fact and implemented a facile template-driven synthesis of lead halide perovskite nanocrystals in hexagonal mesoporous silica. ${ }^{[492]}$ The investigators impregnated the pores of various commercial mesoporous materials, such as MCM-41 and SBA-15, with perovskite precursor solutions and, after drying, they observed the formation of arrays of perovskite nanocrystals compatible with the pores size of the adopted template. The confined nanoparticles showed photoluminescence properties dependent on the pore-diameters of the template and better processability with respect to non-templated nanocrystals. ${ }^{[492]}$

Despite the difficulty of tuning simultaneously the size, composition and uniform dispersion of the encapsulated clusters, also MOFs frameworks have been used as confining matrices for nanoparticles of various nature, including quantum dots. ${ }^{[493,494]}$ For example, strategies for the controlled incorporation of capped nanoparticles of various shape, composition and size into a ZIF can produce composites with good catalytic, magnetic and optical properties derived from the encapsulated nanoparticles. ${ }^{[495]}$ Another emerging strategy is the use of atomic layer deposition approaches. ${ }^{[496,497]}$ Self-assembly methods were successfully exploited to confine nanowires with electron donor character into the periodic pores of electron-poor MOFs acting as acceptors.
This strategy has led to donor-acceptor hybrid materials composed by arrays of semiconductive nanowires incorporated in semiconductive MOF frameworks (Figure 30b). ${ }^{[498]}$

a)
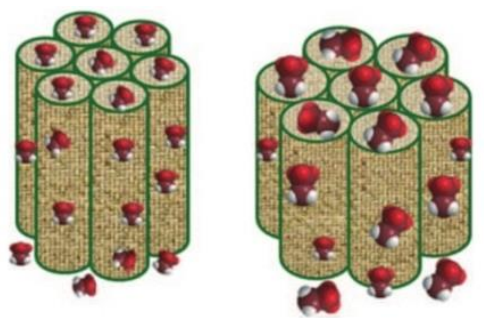

b)

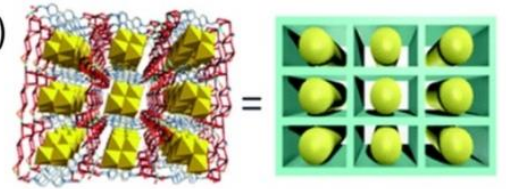

Figure 30. Confined nanostructures in mesopores and MOFs: a) $\mathrm{Fe}_{2} \mathrm{O}_{3}$ nanoparticles of uniform sizes fabricated inside mesoporous supports of tunable diameter [Reproduced from Ref. ${ }^{[489]}$ with permission from WILEY-VCH]. b) semiconducting iodoplumbate nanowires (in yellow) regularly organized in the pores of semiconductive pyridinium MOF [Reproduced from Ref. ${ }^{[498]}$ with permission from The Royal Society of Chemistry]

\section{Supramolecular organization of water and cations in confined spaces}

Mainly because of their impact on catalysis, the location and distribution of extraframework cations in zeolites has been object of many experimental[499-504] and computational[505-510] studies. In both hydrophilic or hydrophobic frameworks, water behavior has been intensively explored, ${ }^{[217,511-518]}$ uncovering unexpected supramolecular structures, such as nanohelices (in natrolite) ${ }^{[511]}$, triple helices (in VPI-5), ${ }^{[87,519]}$ cyclic hexamers (in $\mathrm{NaY}$ supercages), ${ }^{[520,521]}$ or nanoworms (in hydrophobic silicalite, Figure $5^{[522,523]}$ ) along with the more common nanospheres (in zeolite A) ${ }^{[524]}$ and ice-like nanotubes (in $\mathrm{AlPO}_{4}-5$ and SSZ-24). ${ }^{[525]}$ Overall, the message is that water behavior differs significantly in passing from hydrophobic to hydrophilic zeolites (Figure 31).

Water molecules can be introduced in hydrophobic (silica-rich) zeolites by a moderate pressure; once entrapped, they mainly form hydrogen bond among themselves and interact only weakly with framework walls. Such weak interactions are actually nonnegligible, as they perturb the hydrogen bonding network and dynamics. ${ }^{[523]}$ However, as a first approximation, water aggregates in hydrophobic zeolites might be considered similar to "isolated" water clusters or droplets, depending on the size of the channel. ${ }^{[518,526,527]}$ Hence, the structure of hydrophobically confined water aggregates is mainly determined by the geometric shaping effect of the channels and cages of the confining matrix. 


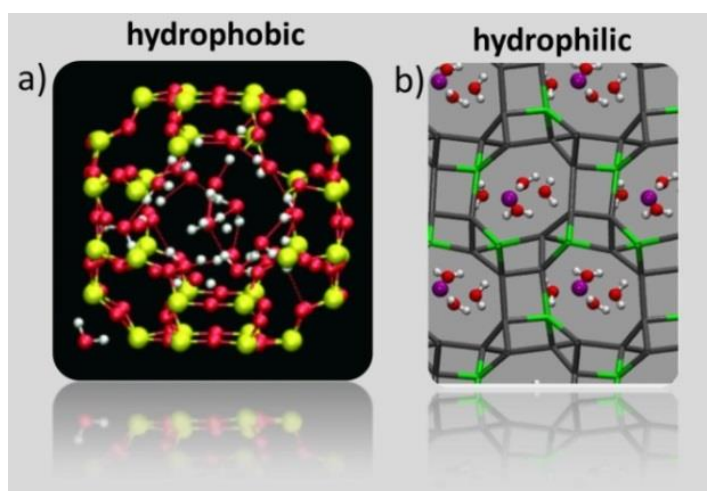

Figure 31. The different organization of zeolitic water in a) hydrophobic and b) hydrophilic frameworks. a) Water in the sodalite cage of all-silica LTA: the leading interaction is water-water hydrogen bonding. Colors: $\mathrm{Si}=y e l l o w, \mathrm{O}=$ red, $\mathrm{H}=$ white [Adapted from Ref. ${ }^{[526]}$ with permission from WILEY-VCH]. b) Water and $\mathrm{Ca}^{+}$cations in natural yugawaralite: $\mathrm{Ca}^{+}$are coordinated to Al-bonded framework oxygens and water, and water molecules form hydrogen bonds with themselves and $\mathrm{Al}$-bonded framework oxygens. Colors: $\mathrm{Ca}=$ purple, $\mathrm{Si}=g r e y$, $\mathrm{Al}=$ green, $\mathrm{O}=\mathrm{red}, \mathrm{H}=$ white. Framework oxygens and hydrogen bonds are not shown for clarity. [Illustration created with VMD; X-ray positions of Ref. ${ }^{\left[{ }^{[28}\right]}$ ]

On the contrary, in hydrophilic frameworks, the interaction of water with charge balancing cations and framework oxygens can be strong and specific. Along with the water-water interactions, it governs the organization of the confined species. Water and ions in hydrophilic cavities cannot be regarded as "isolated species"; they form rather, jointly with the framework, a supramolecular structure extending in the three dimensions.

The fascinating patterns of water and cations in natural zeolites are often very difficult to study by diffraction: the extraframework sites have low occupancy, and multiple symmetry-related positions are possible. ${ }^{[57,106,107,502,529-531]} \mathrm{A}$ further obstacle is the possible co-presence of two crystalline phases, like the tetragonal and monoclinic polytypes of tschernichite, the natural analog of zeolite beta (Figure 32). ${ }^{[529]}$ In large-pores zeolites (like mazzite, Figure 32 ) it is very challenging to locate all the water molecules, even with the knowledge of Al preferential location (obtained via ${ }^{27} \mathrm{Al}$ SS-NMR). ${ }^{[532]}$ Yet those averaged positions give hints on the arrangement of the guests, and if they are represented together (Figure 32), the striking complementarity between framework and confined species emerges. This relation is more profound than a simple geometric effect dictated by shape-volume constraints, as framework and guests are bound together by a complex network of electrostatic, coordinative and hydrogen bond interactions which underlies the impressive stability of natural zeolites. ${ }^{[126]}$

One way to get insight on the responsivity properties of the confined guests' assemblies is to study their behavior under an external perturbation, such as temperature or pressure. Whereas an increase of temperature causes greater disorder in the hydrogen bond network, and then dehydration, pressure effects are less straightforward.

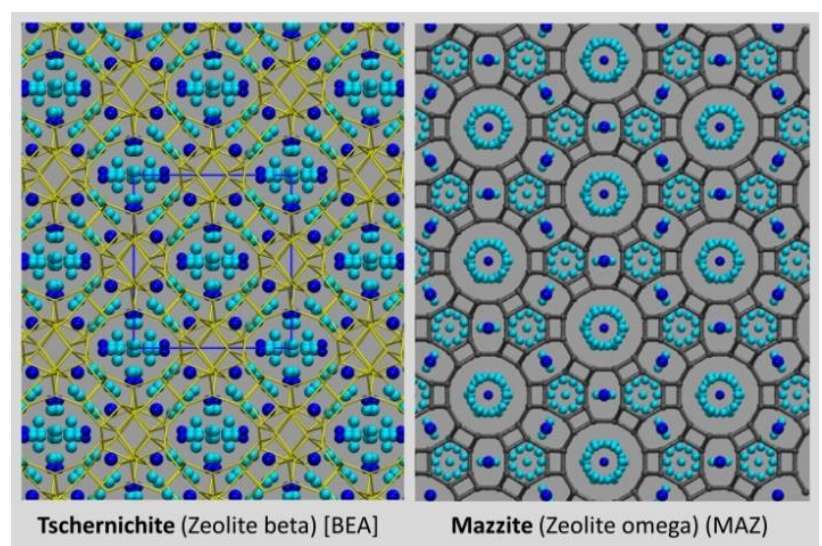

Figure 32..Nature-made supramolecular organization: Framework structures of the natural zeolites tschernichite (BEA) ${ }^{[529,533]}$ and mazzite (MAZ) ${ }^{[532]}$ (and of their respective synthetic counterparts (in parentheses)). The colored balls show the water and cation sites (with fractional occupancy). The blue solid lines are a guide for the eye and illustrate the unit cell, as exemplified for BEA. [Illustration created with VMD, using the X-ray positions from Refs ${ }^{[529,532]}$.]

When zeolites are compressed hydrostatically (with nonpenetrating media) ${ }^{[53,534-537]}$ the water-cations arrangement changes responsively. Also, the framework undergoes the energetically cheapest change: without internal distortion, the tetrahedral units rotate around their hinges and the channels deform. ${ }^{[535,538,539]}$ The guest species play a crucial role in the deformation of the zeolite framework. ${ }^{[53,536]}$

Under high pressure, the formation of water dimers in scolecite triggers a phase transition, ${ }^{[540]}$ and the coordination network of the cations guide, as a sort of template, the distortions of the framework, as exemplified by calcium in natural zeolite yugawaralite. ${ }^{[528,541]}$ Such a "template" role of the extraframework cations also explains the resistance to dehydration of tightlycoordinated zeolitic water, ${ }^{[107]}$ and may be visually captured by Figure 33, showing how precisely the naturally confined species, taken as a whole, fill the network of empty spaces. Interestingly, a similar template role of the guest molecules has been first proposed for clathrasils. The pressure-induced amorphization of dodecasil-3C (characterized by cage voids), and decadodecasil3R (with 2D-channels) was studied by IR spectroscopy, X-ray diffraction and molecular dynamics. Amorphization was found to be fully (dodecasil-3C) or partially (decadodecasil-3R) reversible only when guest molecules were present. ${ }^{[542]}$ Hence, the guests acted as "organizing centers", making the lattice resilient to much higher pressures compared to guest-free clathrasils. ${ }^{[542]}$

A surprisingly simple architecture of water and cations is hosted inside a mineral called bikitaite. ${ }^{[543]}$ This rare zeolite hosts in its 1D-channels linear assemblies of water molecules coordinated to lithium cations (Figure 34). Such an arrangement was termed "one-dimensional ice"[544,545] because of its solid-like behavior, characterized by impressive stability at high temperature ${ }^{[546]}$ or high pressure ${ }^{[547-550]}$ conditions, and no translational/rotational motions of the individual molecules irrespectively of the $\mathrm{Al} / \mathrm{Si}$ ordering in the framework. ${ }^{[545]}$ This knowledge was obtained by multitechnique analyses including computational studies ${ }^{[230,545,551,552]}$ neutron-[543] and $X$-ray diffraction, ${ }^{[544]}$ single crystal polarized Raman, ${ }^{[553,554]}$ and ${ }^{1} \mathrm{H}$ SSNMR relaxation experiments. ${ }^{[555]}$ 
The template effect of confined species in natural zeolites

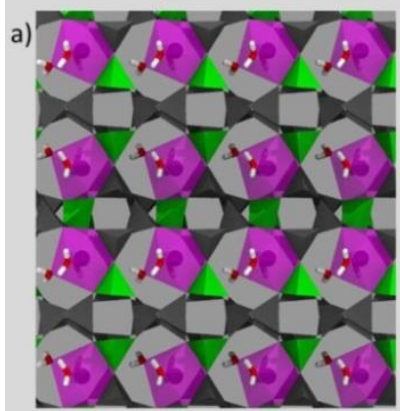

Yugawaralite [YUG]

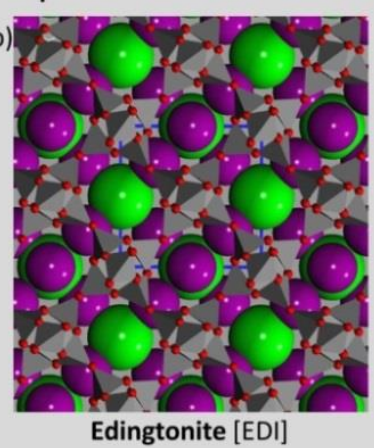

Edingtonite [EDI]

Figure 33. The organization of extraframework species in natural zeolites a) yugawaralite and b) edingtonite. a) The coordination polyhedron formed by the calcium cations (purple) is linked to the negatively charged $\mathrm{AlO}_{4}$ units (green). $\mathrm{SiO}_{4}$ units are in grey, water molecules in red and white (framework oxygens are not shown). b) $\mathrm{Cl}$ (green) and $\mathrm{Ba}$ (purple) alternatively occupy the edingtonite cage. Tetrahedral atoms $\mathrm{Si}, \mathrm{Al}$ are represented in grey, framework oxygens as red little balls (water molecules are not shown). [lllustration created with VMD, using the X-ray positions from Ref. ${ }^{[528]}$ (YUG); Ref. ${ }^{[58]}$ (EDI).]

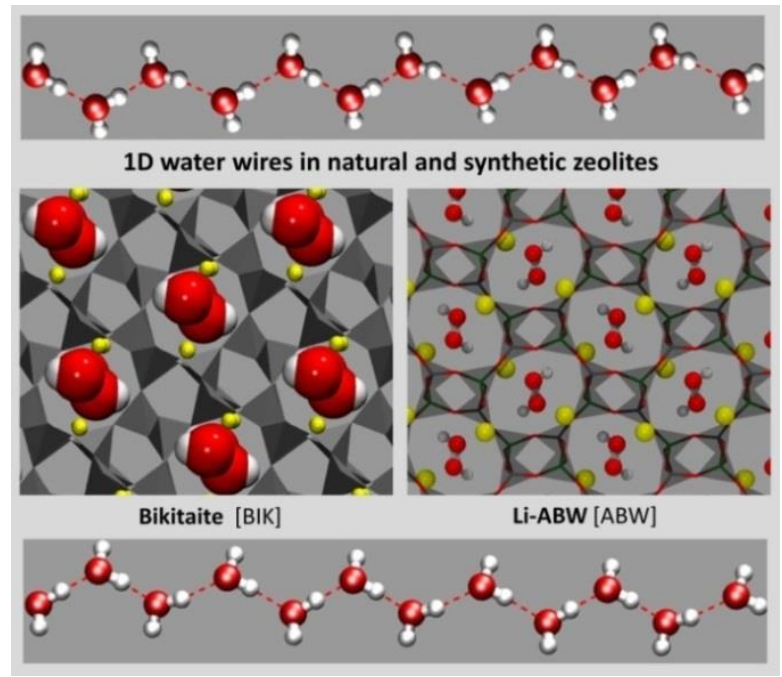

Figure 34. Water wires occupy the 1D-channels of natural bikitaite (left) and synthetic zeolite Li-ABW (right): water molecules are coordinated to $\mathrm{Li}^{+}$(yellow balls) and do not form hydrogen bonds with the framework oxygens. The two zeolites (and the water wires) are viewed along the channel direction. In-plane views of the water wires are shown in the top and bottom panels. [Illustration created with VMD, with X-ray positions from Ref. ${ }^{[544]}(\mathrm{BIK})$ and Ref. ${ }^{[556]}(\mathrm{ABW})$ ].

The latter ones, performed in the temperature range $224-418 \mathrm{~K}$, identified one single relaxation process: a $180^{\circ}$ flip-flop motion of water exchanging the positions of its protons, ${ }^{[555]}$ analogous to the librational twist of water in solid hydrates. ${ }^{[557]}$ Such water flipping was reproduced by molecular dynamics simulations, ${ }^{[551]}$ that, in line with NMR, did not observe any water diffusion in that temperature range. Other modeling studies proposed a two-step dehydration mechanism: the first and rate determining one is water escape from its site, followed by a fast jump to an empty site. ${ }^{[546]}$ The $\mathrm{Li}$ cations assist water migration, lowering the free energy barrier for the jump. ${ }^{[546]}$ Such a single-file diffusion in 1D channels can occur only if one water site is empty, i.e. if the water wire has "defects“.

Notably, water in bikitaite is not hydrogen bonded to framework oxygens, but interacts with $\mathrm{Li}^{+}$via strong coordination bonds (Figure 34). Also the synthetic zeolite Li-ABW has 1D channels, where water chains are confined. ${ }^{[558-560]}$ Yet such water wires are

easily perturbed upon compression, because water forms hydrogen bonds with framework oxygens and the chain is gradually disrupted with increasing pressure. ${ }^{[223]}$

The water chains in the two zeolites have different behavior because the framework electric dipole is much stronger in bikitaite compared to $\mathrm{Li}-\mathrm{ABW}$, and this implies a greater electrostatic stabilization to the water architecture. ${ }^{[558]}$ Also importantly, the chain does not form when $\mathrm{Li}$ is replaced by $\mathrm{Na}$, as demonstrated for the zeolite $\mathrm{Na}-\mathrm{ABW}$, because of the lower polarizing power and binding capacity of the sodium cation towards water and framework oxygens. ${ }^{[559]}$ This example evidences the delicate interplay of host-guest interactions in hydrophilic zeolites: fine differences in the framework structure, or in the nature of the charge balancing cations, may affect significantly the stability and dynamic behavior of the confined supramolecular assembly. These factors also govern the organization of more complex guests such as lanthanides, ${ }^{[51]}$ metal complexes, ${ }^{[561,562]}$ or dyes, inside hydrophilic hosts. ${ }^{[563-566]}$

\section{Organization of dihalogens in 1D channels}

The environmental relevance of toxic gas capture[46] and the electric conductivity enhancement caused by iodine uptake ${ }^{[567,568]}$ have raised great interest on the incorporation of dihalogen molecules in diverse hosts like clathrasils, ${ }^{\left[{ }^{[24,569]}\right.}$ zeosils, ${ }^{[46,569-571]}$ and ALPOs, ${ }^{[572-575]}$ but also organic salts, ${ }^{\left[{ }^{576]}\right.}$ organic zeolites, ${ }^{[567]}$ Hofmann clathrates, ${ }^{[577,578]}$ and other MOFs. ${ }^{[579-581]}$

The structure and composition of the host determine the intermolecular interactions responsible of the capture/release mechanisms. In various MOFs, for instance, iodine capture is based on halogen bond interactions with metal centers or building blocks of the framework, which often produce (poly)iodide moieties. ${ }^{[576,577,581]}$ In particular, a recent single-crystal X-ray study showed that $\mathrm{I}_{2}$ molecules were incorporated formally as $\mathrm{I}_{4}{ }^{2-}$ anions in a Cu-MOF thanks to strong $\left.|-\cdots|_{2} \cdots\right|^{-}$interactions with the $\left\{\mathrm{Cu}_{4} \mathrm{I}_{4}\right\}_{n}$ chains of the host. Notably, a strong conductivity increase was detected upon $\mathrm{I}_{2}$ uptake, suggesting a possible use of the material in $\mathrm{I}_{2}$-electric sensing. ${ }^{[568]}$

In zeosils and ALPOs, dihalogens interact more weakly with the host and are incorporated formally as neutral species. In this case the arrangement of the guests is stabilized also by homo-halogen interactions, recently evidenced for $I_{2}$ clusters in superfluid helium droplets. ${ }^{[582]}$ Dihalogens form intriguing supramolecular structures, like wires of aligned $I_{2}$ molecules, ${ }^{[573]}$ inside zeosil or ALPO cavities. These hosts act as templates, because they control via their void spaces the interactions among confined dihalogens. ${ }^{[569]}$ This effect was evidenced by the colors of the $I_{2}$ inclusion compounds, ranging from violet to red-brown depending on the dimensionality of the host. ${ }^{[569]}$ The violet color, typical of gasphase $I_{2}$, was found in DDR clathrasil, indicating isolated molecule behavior, while $I_{2}$ in TON zeosil was blue-violet due to the halogen-bonded chains in the host 1D-channels. ${ }^{[569]}$ The color of the compound $\mathrm{I}_{2}$.FER (2D-channels) suggested co-presence of iodine chains and isolated molecules. Finally, inclusion in the MFI 3D-channels resulted in a red-brown color, ${ }^{[569,571]}$ ascribed to stronger halogen interactions. ${ }^{[569]}$ Several studies have then focused on the organization of confined dihalogens, exploring not only the structure of the aggregates, but also how their organization is affected by size and concentration of the guests, ${ }^{[583]}$ structure and composition of the host, ${ }^{[574,583]}$ solvation, ${ }^{[584]}$ temperature, ${ }^{[585-587]}$ and pressure. ${ }^{[588-590]}$ In general, molecules confined in small cages, like in SOD (Figure 27), undergo rotational motion - which may be more frictioned for large 
guests, but is normally very fast. ${ }^{[591]}$ Hence, geometrical match between the host cavities and the guest is not sufficient to achieve orientational ordering because of the thermal motion. This happens also to dihalogens in the 1D channels of AEL and AFI aluminophosphates:[572,573,575,584,586,588,592] rotational barriers are greater in the AEL elliptical channels, featuring an alternation of wide and narrow regions (Figure 35). Raman spectroscopy revealed that the confined dihalogens could orient either aligned or perpendicular to the channel axis, as the two orientations give rise to distinct Raman bands. ${ }^{[588,589]}$ The lower frequency band is due to wires of aligned molecules: indeed, the inter-halogen interactions weaken the intramolecular bond, causing a decrease of its force constant. ${ }^{[569]}$ As the aligned halogen wires are normally interrupted by perpendicular molecules, only short chains are formed. For example, Liu et al. introduced $\mathrm{Br}_{2}$ vapor under vacuum in the round 1D-channels of AIPO4-5 (AFI framework type) to realize stable $\mathrm{Br}_{2}$ arrays. ${ }^{[586]}$

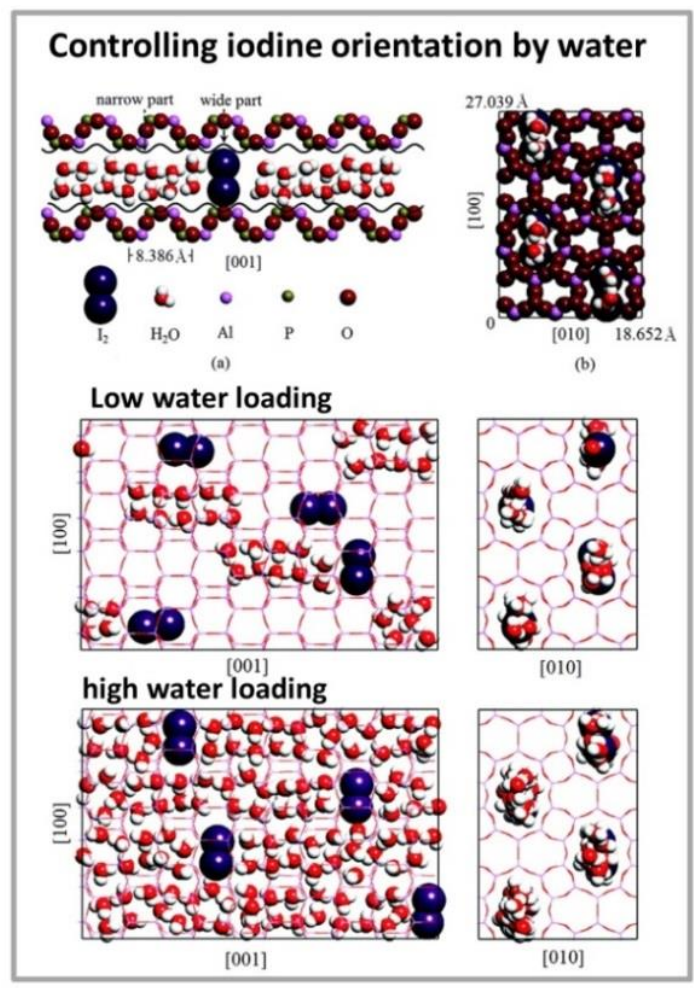

Figure 35. The role of water in the organization of iodine inside the $1 \mathrm{D}$ channels of ALPO4-11 (AEL). The top panel represents: a) an $\mathrm{l}_{2}$-containing channel ( $\left.I_{2} @ A E L\right)$ at high water loading. The channel features an alternation of wide and narrow parts with a periodicity of $1 / 2 c(c=8.386 \AA$ is the AEL lattice parameter along the channel axis, while the size of the narrow region is $4.3 \AA$. $6.1 \AA$ ). b) Projection on (110) plane. The middle and bottom panels show 2 configurations (viewed in the (101) and (110) planes) from molecular dynamics simulations of $\mathrm{I}_{2} @ A E L$ at low and high water loading, respectively. [Adapted with permission from Ref. ${ }^{[584]}$ Copyright 2012 American Chemical Society.]

Raman spectra evidenced that the $\mathrm{Br}_{2}$ chains were nearly aligned to the channel axis of AFI crystals, and that a significant proportion of molecules were parallel to the channels. ${ }^{[586]}$ Notably, the population of aligned $\mathrm{Br}_{2}$ was increased by operating either at high pressure ${ }^{[590]}$ (see chapter 8 ) or at low temperature. ${ }^{[586]}$

To further explore thermal effects, Chen et al. studied the confinement of iodine molecules in $\mathrm{AFI}^{[587]}$ and $\mathrm{AEL}^{[585]}$ frameworks down to $-196^{\circ} \mathrm{C}$. Combined Raman experiments and molecular dynamics indicated that, upon decreasing temperature, the thermal motion of the confined molecules slowed down, thus inducing a change in orientation. The population of $\mathrm{I}_{2}$ molecules aligned to the channel increased: hence, longer $I_{2}$ chains were formed in both hosts, with higher ordering. Nonetheless, such a cooling-induced order enhancement was not preserved after recovery to room temperature. ${ }^{[585,587]}$

The reversibility of the iodine orientation inside ALPO channels is mainly due to the guest-host interactions being noncovalent, nonspecific, and weak. What happens now if such molecules are forced to share the channel space with another guest - water, for example. In this case, the orientation depends on the water content: at low water loading $\mathrm{I}_{2}$ is mainly aligned to the axis, while at high loading the orientation is perpendicular (Figure 35). ${ }^{[584]}$ The water-solvated iodine standing in the widest part of the channel is energetically the most favorable state of the system at room temperature and full hydration, ${ }^{[584]}$ because at such conditions water hydrogen bonding becomes the dominant interaction. The uniform "standing" orientation of $I_{2}$ (Figure 35) is a signature of the organizing role of water, which also affects the orientation (and optical anisotropy) of luminescent guests in zeolite-based antenna systems, as discussed in chapter 7. ${ }^{[593-596]}$

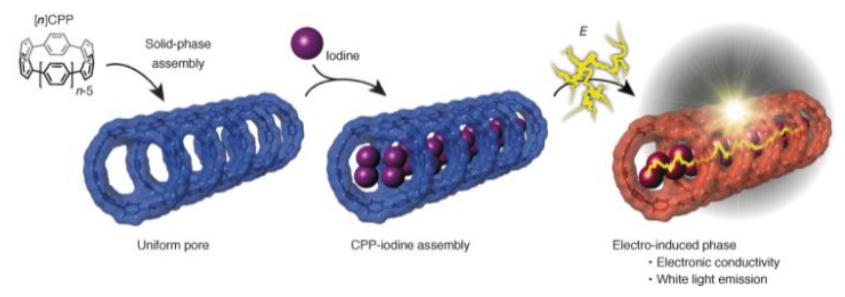

Figure 36. 1D lodine chains confined in an organic nanocylinder made by cycloparaphenylene (CPP) rings. Electric stimulation of this host-guest system generates electronic conductivity (from iodine chains) and white light emission (from the organic matrix).[Reprinted with permission from Wiley- $\mathrm{VCH}$. $]^{[597]}$

Beside interesting structural aspects, dihalogens, when forced into nanosized spaces, may reveal unexpected behavior, especially when the confining matrix is stimuli-responsive. One such matrices, formed by condensed organic rings, contains $I_{2}$ chains similar to those found in zeolites. ${ }^{[597]}$ Upon activation with an electric field, the system displayed electronic conductivity, ascribed to polyiodide chains formed by charge transfer from the organic matrix (Figure 36). Although the polymerization process has not been clarified yet, the conductivity was found to depend on the size of the ring. This suggests that the dynamics of confined $\mathrm{I}_{2}$ molecules may play a relevant role in the process. ${ }^{[597]}$ Technologically appealing properties may also appear if iodine is confined in zeolites, for example in the familiar silicalite (Figure 5), where $\mathrm{I}_{2}$ forms a semiconducting 3D-supramolecular network (Figure 37). ${ }^{[46]}$ Indeed, the outstanding $\mathrm{I}_{2}$ sorbent properties detected for this highly hydrophobic MFI were ascribed to the scarcity of framework defects and the ability to tightly fit $\mathrm{I}_{2}$ within the channels by forming a confined 3D-network of iodine. ${ }^{[46]}$
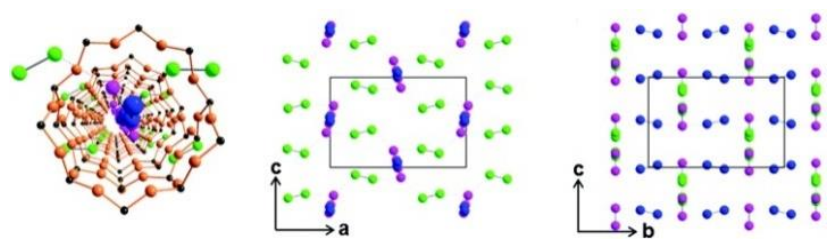

Figure 37. Semiconducting $I_{2}$ network in silicalite. (left) Vision along the $b$ axis of hydrophobic silicalite channels containing 3 types of crystallographically different $I_{2}$ molecules. A row of iodine molecules is parallel to the $b$-axis (in blue). 
The full 3D-network of confined $\mathrm{I}_{2}$ molecules, viewed in the ac plane (center) and in the bc plane (right). [Reproduced from Ref. ${ }^{[46]}$ with permission from The Royal Society of Chemistry]

Confined chlorine molecules do exhibit interesting behavior as well. For example, although the first excited state of gas-phase $\mathrm{Cl}_{2}$ is a triplet, experiments on confined $\mathrm{Cl}_{2}{ }^{[598]}$ suggest that the excitation should occur on an unbound singlet state relatively close to the triplet, causing thus the dissociation of the molecules.

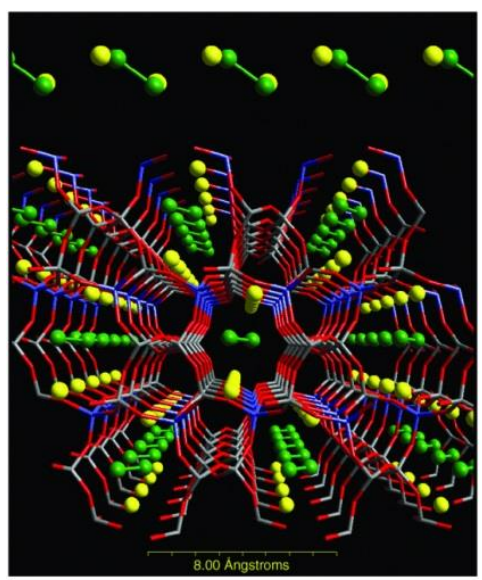

Figure 38. Linear organization of chlorine molecules in the 1D channels of zeolite bikitaite. Color codes: $\mathrm{Cl}=$ green, $\mathrm{Li}=$ yellow, $\mathrm{Si}=$ grey, $\mathrm{Al}=$ blue, $\mathrm{O}=$ red. [Reproduced from Ref. ${ }^{[599]}$ with permission from WILEY-VCH].

By first principles modeling, ${ }^{[599]}$ Medici et al. simulated a similar process, namely the electronic excitation of a $\mathrm{Cl}_{2}$-wire confined in the 1D-channels of bikitaite (Figure 38). ${ }^{[600]}$ Zeolite confinement, and the organization of $\mathrm{Cl}_{2}$ in supramolecular chains, favor collisions of an excited molecule with the adjacent ones, triggering the excitation-transfer. Indeed, the calculations showed that the excitation was localized on a single $\mathrm{Cl}_{2}$ molecule, which dissociated into two neutral $\mathrm{Cl}$ atoms. Collision of such atoms with the adjacent molecule in the wire led to excitation transfer; as a result, another molecule dissociated and a new $\mathrm{Cl}-\mathrm{Cl}$ bond was formed. The excitation transfer occurred at picosecond-scale via a Dexter ${ }^{[601]}$ mechanism, and may be viewed as a displacement of the $\mathrm{Cl}-\mathrm{Cl}$ bonds along the supramolecular chlorine wire. ${ }^{[599]}$ Such insight could be of help for understanding electronic excitation transfer along ordered arrays of confined molecules.

\section{Organization of complex species in 1D}

Linear molecules, even if confined in 1D-systems, in general do not form perfectly ordered 1D structures, especially when the size of the channel is large enough to allow guest species to rotate. Yet confinement in one dimension still remains the easiest solution to the organization problem, because: i) the number of possible arrangements is limited, and ii) the organization process may be more easily designed and controlled.

For this reason, one-dimensional porous matrices have been heavily exploited to confine any possible kind of system of nanoscopic size, from inorganic to organic, biological and hybrid bio-inorganic: fullerenes were included in mesoporous oxides, ${ }^{[602]}$ MOFs, ${ }^{[31,419]}$ or COFs $;{ }^{[421]}$ natural pigments like chlorophyll, ${ }^{[603]}$ or light-harvesting complexes like LH2 were confined in mesoporous silica, ${ }^{[604,605]}$ and extra-large channel MOFs were invented to encapsulate enzymes (Figure 39). ${ }^{[606]}$ a)

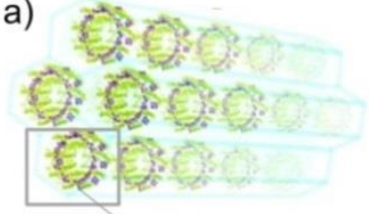

b)

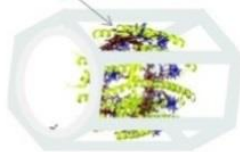

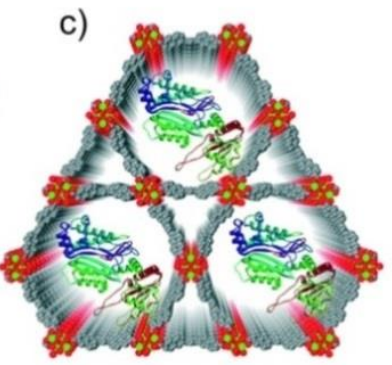

Figure 39. Mesochannels for 1D organization of huge molecules. a) Inclusion composite of photosynthetic complex LH2 in mesoporous silica; b) zoomed view of a confined LH2 molecule. [Adapted by permission, Ref. ${ }^{[604]}$ Copyright 2006 American Chemical Society]. c) Schematic view of enzymes in MOF-channels. [Reprinted from Ref. ${ }^{[606]}$ by permission from The Royal Society of Chemistry]

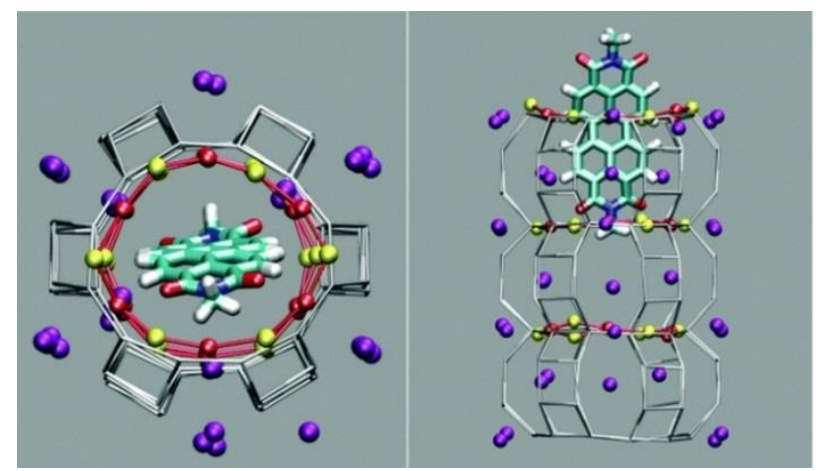

Figure 40. Difficult entrance of a guest in a nanochannel. If the van der Waals size of the guest is slightly greater than the pore opening, both the host matrix and the guest deform cooperatively in the penetration process. ${ }^{[607]} \mathrm{Here}$, the guest is a perylene-diimide dye and the host is zeolite L (LTL-type ${ }^{[58-60,608]}$ ). The channel entrance becomes transiently elliptical when the dye passes through. This process occurs at high temperatures thanks to the favorable interaction of the carbonyl group of the dye with the zeolite $\mathrm{K}^{+}$cations, and to host-guest cooperative vibrational motions. The motion of the dye inside the porous matrix has an asymmetric free energy profile, ${ }^{[607]}$ which makes the entrance process favored over the exit, in analogy to what observed for the passage of a diazobenzene dye through a crown-ether ring. ${ }^{[609]}$ Color code: Si and Al atoms of zeolite $\mathrm{L}$ framework = gray sticks. $\mathrm{C}=$ cyan; $\mathrm{H}=$ white; $\mathrm{N}=$ blue, carbonyl $\mathrm{O}=$ red; $\mathrm{K}^{+}=$purple. The red and yellow balls (framework oxygens) on channel aperture highlight the deformation of the opening at the passage of the molecule,

The dimensions of the pore openings are an extremely important parameter, because they dictate the size of the molecules that may enter the pores and be organized within their spaces. However, molecules are flexible, and can often manage to pass through the pore orifices even if they have nominally a larger size. Framework flexibility, which is a distinctive feature of MOFs such e.g. ZIF-8, ${ }^{[423-425,610,611]}$ may also play a key role in facilitating the penetration of oversized guests via a window-swing effect. ${ }^{[425]}$ Contrary to common expectations, also zeolites manifest a surprising structural flexibility, which allows for the vapor-phase incorporation of relatively bulky molecules (e.g. perylene-diimide) in nanosized channels like those of zeolite L (Figure 40). ${ }^{607]}$

Despite all progress in the fabrication and characterization of channel-based materials, the incorporation mechanisms and the host-guest interactions determining the composites' properties are still little understood, often because of the complexity of both encapsulated species and confining matrix. One remarkable 
exception is the family of composites produced by the inclusion of chromophores in zeolite $L^{[59,608]}$ (LTL topology). The fabrication of these hierarchically organized photoactive materials and their use as artificial antenna system, pioneered by the Calzaferri group $^{[29,36,39,49,563,612-618]}$ has been thoroughly investigated and the acquired knowledge played a seminal role, for example, in extending their scope to biomedical uses. ${ }^{[619-624]}$ Also importantly, dye-zeolite $L$ hybrids stimulated the quest of new host matrices for luminescent supramolecular systems: not only zeolite frameworks of diverse shape and composition, ${ }^{2688,625-632]}$ but also mesoporous silicas/organosilicas, ${ }^{[63,365,376,633-636]}$ 1D macrocycle channels, ${ }^{[38,637]}$ and MOFs. ${ }^{[62,64,638-647]}$

\subsection{Dye-zeolite $L$ materials as artificial antenna prototypes}

Zeolite L (Figures 40, 41) is a convenient host system because the size and shape of its one-dimensional channels allow for the encapsulation of a broad variety of dyes, either in neutral or in cationic forms. In this way, one can obtain guest-host composites with remarkable organization patterns, up to end-to-end sequenced chains of chromophores. ${ }^{[29,36,40]}$
Other advantages are the facile synthesis of zeolite crystals with tailorable aspect-ratio ${ }^{[648-650]}$ and the selective functionalization of their external surfaces, either at the base (channel entrances) or at the coat region. ${ }^{[616]}$ These features allow to obtain zeolite crystals self-assembled via coordinative linkers, ${ }^{\left[{ }^{[61]}\right.}$ arranged in close-packed monolayers, ${ }^{[652]}$ integrated with devices, ${ }^{[61,653]}$ and even connected with living systems such as bacteria, ${ }^{[654]}$ which can then be targeted and killed by red-light irradiation. ${ }^{[655]}$

Nonetheless, the atomistic structure of these versatile compounds often remains unexplored, as suggested by the scarce number of diffraction studies on dye-zeolite hybrids. ${ }^{[133,656-664]}$ Fortunately, computational modeling comes to the rescue. ${ }^{[171]}$ Theoretical approaches have proved especially useful to study processes at the channel entrances (Figure 42), which govern the formation of the organized material[607] and its potential practical uses. ${ }^{[191]}$ Channel entrances are particularly important because they can be functionalized; for example, with stopper molecules (traditionally named "stopcocks"), that, by preventing the leakage of the chromophores and the entrance of undesirable species, ${ }^{[665]}$ preserve the inner supramolecular organization and functionality of the material (Figure 42). ${ }^{[615,666,667]}$

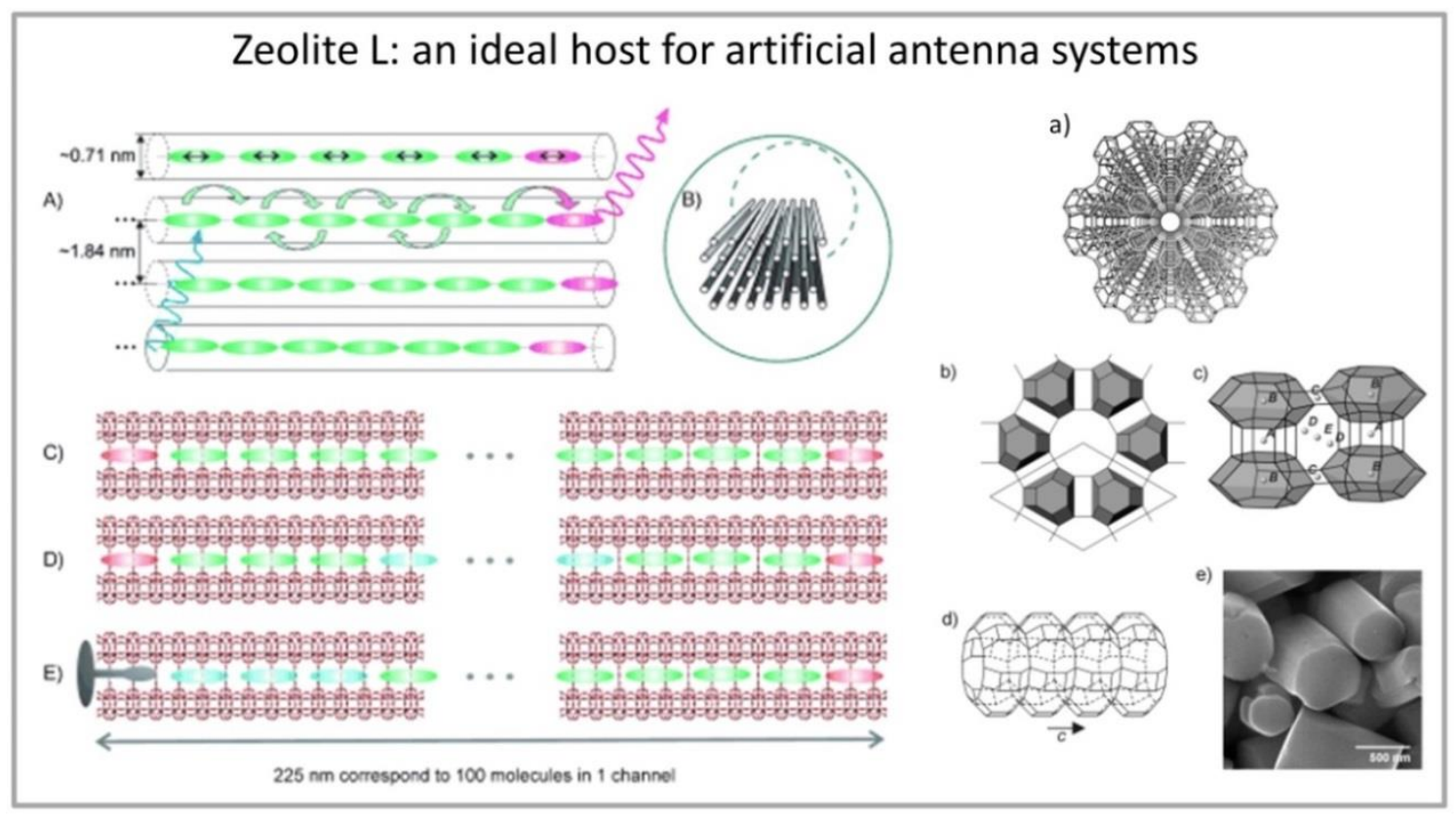

Figure 41. Artificial antenna systems based on zeolite L are illustrated on the left panel. A) Donor dyes (green) absorb light and transfer excitation energy by Förster resonance energy transfer (FRET) to acceptor dyes (magenta) at the channel end. Electronic transition dipole moments (ETDM) of the dyes are indicated by double arrows. B) Arrangement of parallel nanochannels. C,D) Sequential organization of two- (C) and three- (D) types of chromophores. E) Unidirectional antenna system: energy is transported only one-way, from left to right. The left side of the channel is blocked by a molecular stopper ("stopcock"), ${ }^{[666]}$ represented in gray. Commonly adopted perylene-diimide dyes ${ }^{[668,669]}$ are about $2.25 \mathrm{~nm}$ long (see e.g. Ref. ${ }^{[665]}$ ). [Reproduced from Ref. ${ }^{[653]}$ with permission from WILEY-VCH]. The zeolite $L$ framework is shown on the right panel: a) view along the channel axis (corresponding to the $c$ axis); b) the cancrinite cages (shown as polyhedra), c) the different positions of extraframework cations (A to E); d) one 12-membered ring channel of zeolite $L$ viewed along the $c$ axis, e) SEM image of hexagonal crystals of zeolite L. [Adapted from Ref. ${ }^{[29]}$ with permission from WILEY-VCH].

The specific modification of the channel openings via the grafting of alkoxysilane moieties (Figure 42) is also fundamental to attain macroscopic organization of zeolite crystals (by covalent attachment to a support, for example $)^{[564,670,671]}$ and to add further functionalities. Typically, the added groups carry magnetic, ${ }^{672,673]}$ or photo/electroresponsive properties - like phthalocyanine dyes, ${ }^{[40,674-676]}$ spiroxanes, ${ }^{[677]} \mathrm{Ru}^{2+[562]}$ and $\operatorname{Ir}(\mathrm{III})^{[678]}$ complexes. Stopcocks can also be used for molecular recognition processes: for instance, the end-modification of zeolite $L$ with adamantane allowed for the selective aggregation of $\beta$-cyclodextrin-coated gold nanoparticles at the zeolite crystal terminations. ${ }^{[679]}$

Cationic or neutral dyes are commonly introduced via ionexchange or vapor phase methods, respectively. ${ }^{[29,680-683]}$ An alternative strategy is the ship-in-a-bottle method, where dyes are incorporated directly in the synthesis step. ${ }^{[564]}$ As shown in Figure 41, the well-defined geometry of the 12-membered ring channels of zeolite $L$ ( $7.1 \AA$ diameter) should force the confined molecules to self-organize in single-file, providing thus an efficient transfer of excitation energy along the chain. ${ }^{[617,653,684]}$ 
Achieving supramolecular organization in zeolite $L$ is however not straightforward. Besides the framework, also size, shape, charge, and concentration of the guests govern the inclusion process. In addition, other species may be present, like water. Nonetheless, experimental and theoretical analyses shed some light on how the host-guest, guest-guest, and guest-water interactions affect supramolecular organization, stability, electronic properties, and optical behavior of the composite, also suggesting possible improvements. The high number of chromophores encapsulated in zeolite $L$ could be classified in two broad groups, namely, cationic and neutral species. While the electrostatic potential of the zeolite plays a key role in the arrangement of cationic dyes, neutral chromophores can form localized, specific and strong interactions with the host, like coordination bonds.

7.2 Neutral dyes: is organization imposed only by the space constraints of the matrix?
Perylene-diimide dyes are widely used in functional materials because of their outstanding optical properties. ${ }^{[668,669]}$ Because of their size, these dyes must align to the channel axis to be incorporated in zeolite L (Figure 43). In this case, the organization is dictated by the space constraints of the matrix, but it is finetuned by the geometry of the dye $;^{[685,686]}$ for example, the spacing between the photoactive perylene cores can be tuned via the choice of end-substituents. ${ }^{[65]}$ Also the dye carbonyl groups are crucial, as shown by a recent study. ${ }^{[133]}$ Indeed, X-ray diffraction, total scattering, IR and UV-vis data combined with modeling revealed that the perylene dye tb-DXP is asymmetrically positioned in the channels in order to optimize, compatibly with the steric constraints, the stabilizing interactions between its carbonyl groups and the zeolite potassium cations. ${ }^{[133]}$ The relevance of the $\mathrm{C}=\mathrm{O} \cdots \mathrm{K}^{+}$interaction (amounting to $\sim 26 \mathrm{kcal} / \mathrm{mol}$ for perylene-diimide) ${ }^{[607]}$ can be better understood by considering a carbonyl dye of smaller size: fluorenone.

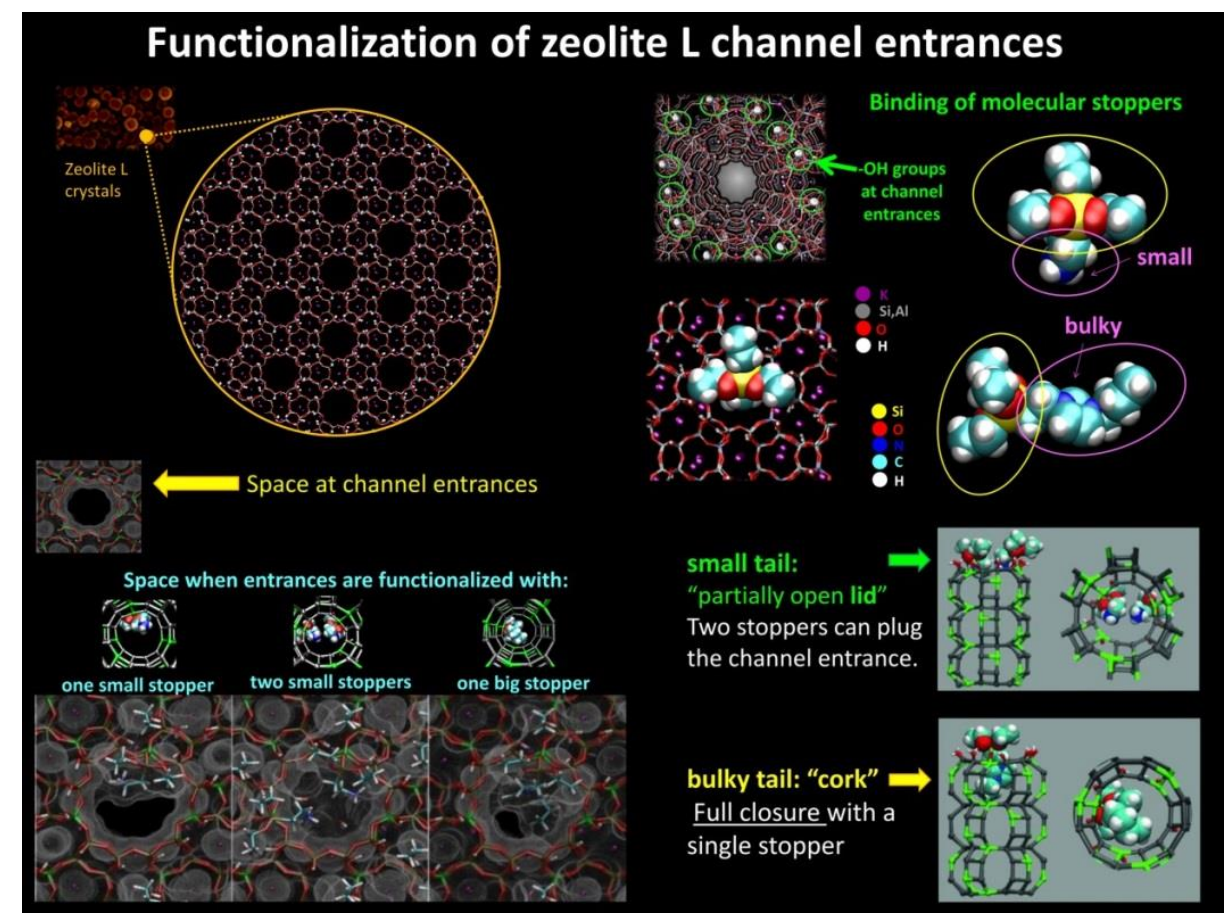

Figure 42. Tuning the pore openings: To master supramolecular organization in zeolites, microscopic insight on the pore entrances is needed. Modeling has given useful information on zeolite $L$ channel entrances and their functionalization with alkoxy-silane moieties, which prevents leakage of guest species. ${ }^{[191]}$ Attachment of these molecular stoppers (or "stopcocks") to the channel entrance modifies the accessibility of the pores according to type and size of the stopper. Bulky stopcocks, with an imidazolium tail group, fully seal the channel entrance acting similarly to a cork on a bottle. [Adapted from Ref. ${ }^{[191]}$ with permission from WILEY-VCH].

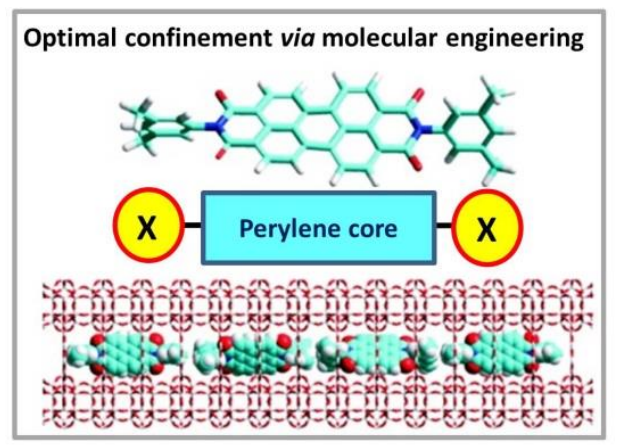

Figure 43: Perylene-diimide (also known as perylene-bisimide) are a family of dyes that fit exactly in zeolite $L$ channels. By the end-substituents (yellow balls), the organization of the dyes and the spacing between the photoactive perylene cores - may be tuned via molecular engineering strategies. ${ }^{[618,665,685]}$ The figure shows, for a typical perylene dye, the structural formula, the schematic representation, and a scheme of the organization of the confined chromophores. Color codes: $\mathrm{C}=$ cyan, $\mathrm{N}=$ blue, $\mathrm{O}=\mathrm{red}, \mathrm{H}=$ white, $\mathrm{Si} / \mathrm{Al}=$ grey. [Adapted with permission from Ref. ${ }^{[685]}$. Copyright 2011 American Chemical Society.]

Due to its size, fluorenone could have some freedom of motion inside the host; yet it forms unexpectedly stable adducts with zeolite $L$ also at humid conditions, ${ }^{[680]}$ because it is bound to the potassium cations via its carbonyl group. Such a strong interaction explains the stability and the optical anisotropy of the composite. ${ }^{[687,688]}$ Indeed, coordination to $\mathrm{K}^{+}$ensures that the fluorenone molecules, on average, are correctly oriented to allow for an efficient FRET. ${ }^{[365,614]}$ 


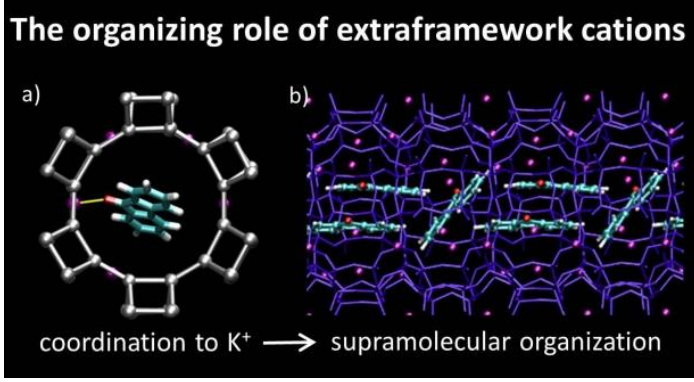

Figure 44. Molecules bearing carbonyl functionalities tend to coordinate $\mathrm{K}^{+}$ when confined in zeolite L. ${ }^{[687]}$ At high loading (b), the extraframework potassium cations cope to induce the self-organization of the dye into a regular, one-dimensional ladder-like arrangements. ${ }^{[660]}$ Color codes: $\mathrm{C}=$ cyan, $\mathrm{O}=$ red, $\mathrm{H}=$ white, $\mathrm{K}=$ purple, Framework=grey (a) or blue (b).

This compound exemplifies how zeolitic extraframework cations are instrumental in directing the supramolecular organization of the confined dyes. Organization does not rely just on steric constraints, rather it is dominated by a specific and strong attractive interaction. ${ }^{[687]}$ When the concentration of the dye increases up to close-packing regime (Figure 44), this interaction still remains important, and drives the formation of a peculiar space-filling arrangement of chromophore molecules. ${ }^{[600]}$ Under compression, this fluorenone-nanoladder, protected by the zeolite host, withstands GPa pressures and responds by approaching with the carbonyl groups the $\mathrm{K}^{+}$cations, i.e., by strengthening the main stabilizing interaction. ${ }^{[664]}$ Such impressive resilience to high pressure is a promising step for extending the application range of dye-zeolite materials beyond normal conditions.

\subsection{Charged dyes. Role of electrostatics, water, and flexibility}

Chromophores bearing a positive charge - from the simplest ones like thionine, ${ }^{[612,629,661,689,690]}$ xanthene dyes, ${ }^{[595,614,648,661,691-693]}$ acridine (and derivatives) ${ }^{[594,596,690,694,695]}$ to more complex molecules like methylviologen ${ }^{[657]}$ or BODIPY-dyes, ${ }^{[40,693,696]}$ - are naturally prone to be incorporated by a zeolite via straightforward ion exchange, a practice which is also used for dye incorporation into anionic MOF nanochannels. ${ }^{[62,64]}$ As a consequence, cationic dyes are very popular as luminescent guests, and their encapsulation has been widely documented by experimental, theoretical, or combined investigations.

Taken as a whole, these studies indicate that cationic dyes normally form stable composites with zeolite $\mathrm{L}$, and are arranged anisotropically inside the channels - which is a necessary condition for energy transfer ${ }^{[29]}$ As for neutral dyes, anisotropy arises because the confined molecules show some preferential orientation due to spatial restraints of the channel and host-guest interactions. ${ }^{[36]}$ Such an orientation is also determined by the electrostatic potential inside the channel, which is especially important for charged guest species, and may become the dominant factor when the composite is dehydrated. ${ }^{[595,596]}$

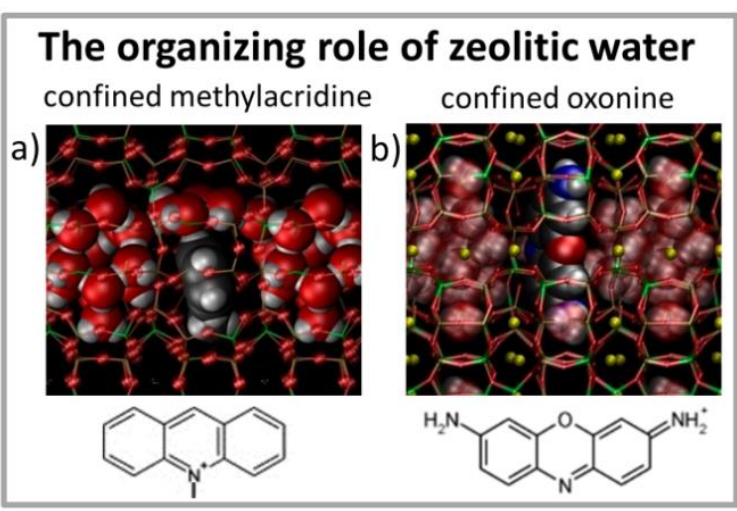

Figure 45. Confined water may impart organization. Structures of hydrated zeolite-L composites containing a) methyl-acridine and b) oxonine dyes. Both dyes are located in the wide part of the channel, with their long axis perpendicular to the channel axis, and surrounded by water. Water molecules are arranged like in perlialite, the natural analog of zeolite L. Colors: $\mathrm{C}=$ grey, $\mathrm{O}=$ red, $\mathrm{H}$ =white, $\mathrm{N}=$ blue, $\mathrm{K}=$ yellow, $\mathrm{Al}=$ green, $\mathrm{Si}=$ brown [in a), $\mathrm{K}$ is omitted for clarity]. [Adapted with permission from a) Ref. ${ }^{[596]}$ Copyright 2013 American Chemical Society; b) Ref. ${ }^{[595]}$ Copyright 2012 American Chemical Society]

That water - normally present in zeolites - could drastically influence both the electronic properties of framework sites, ${ }^{[697]}$ and emission of guest chromophores ${ }^{[593]}$ was long recognized, ${ }^{[698]}$ yet the fundamental role of water in determining the orientation and organization of the dyes in confined spaces was discovered and understood only recently. ${ }^{[595,596]}$ Due to its preference to hydrogen-bond with itself, water tends to confine small dyes into the widest parts of the channel, oriented perpendicular to the channel axis (Figure 45). As in the case of iodine, water has an indirect yet prominent role in the arrangement of small dyes: basically, dye confinement is enhanced by water self-organization in the zeolite environment. Actually, the molecules surrounding the dye feature the same supramolecular arrangement formed by water in perlialite, ${ }^{[57]}$ the natural analog of zeolite $\mathrm{L}$.

To understand and control the organization of confined dyes, we should also recall that neither chromophores, nor the host framework are rigid objects. Various studies disclosed an impressive structural flexibility of the encapsulated species. The confinement in 1-D nanochannels poses severe restrictions to the geometry of the guests: to meet such requirements, molecules may deform spectacularly. Changing conformation is rather easy for methylviologen, ${ }^{[657]}$ or cyanine dyes: ${ }^{[695]}$ they have a single bond, around which the rigid parts of the molecule may rotate, in order to better fit the channels. What it is indeed surprising, is that also fully $\pi$-conjugated chromophores such acridine-, $[596,694,695]$ xanthene- ${ }^{[595]}$ and BODIPY dyes, ${ }^{[696]}$ may exhibit unexpectedly large fluctuations from their gas-phase planar structure (see Figure 46). Also remarkably, such deformations have generally only minor effects on the absorption and emission properties of the dye assembly, and hence on the potential applications of the composites (Figure 46b). ${ }^{[696]}$ Framework flexibility, molecular flexibility and large transient distortions of the molecular geometry might play a pivotal role in favoring the encapsulation and diffusion of the dyes along the channels. For example, flexibility might favor the escape of molecules from "blocking" configurations. The trapping of the dye into such states "plugs" the channel (Figure 46c), as shown by a recent computational study on oxonine in zeolite L. ${ }^{[692]}$ It was found that the "blocking" configurations should be responsible of the so-called "traffic-jam

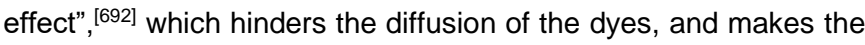
preparation of the composites quite a time-consuming task. ${ }^{[36]}$ 


\subsection{Effects of the framework type}

The above-mentioned dyes, along with several others, have been incorporated in diverse hosts. Inclusion in aluminophosphates yielded, for example, one of the first hybrid composites with laser dyes. ${ }^{\left[{ }^{[69]}\right.}$ More recently, a series of studies on aluminophosphate hosts with different pore size ${ }^{[626-628,700,701]}$ was aimed at finding the framework that would fit "like a glove" to selected guest species (Figure 47). ${ }^{[626]}$ Pyronine $(13.7 \times 6.2 \times 3.2 \AA)$ should be included into the three hosts of Figure 47 always with its main axis nearly parallel to the channel (similarly to perylene dyes in zeolite L), leading to optical anisotropy. Unfortunately, the AFI channels are circular and allow pyronine molecules to pack together because of the favorable guest-guest interactions, forming undesirable $\mathrm{H}$ - aggregates with detrimental effects on luminescence. Things went better with the second host, ATS, whose elliptical channels disfavor the formation of $\mathrm{H}$-aggregates, but not that of close endto-tail arrangements of dyes, called J-aggregates. The third matrix $A E L$, led to composites with the best fluorescence properties, owing to the smaller size of the openings and to the channel topology (Figure 47), that not only matches the geometry of the dyes, but also forces them to be well-separated from each other. ${ }^{[626]}$ By modeling, the investigators also found that acridine fits even better than pyronine into AEL and with more favorable interactions, in keeping with the experimentally found higher loading. ${ }^{[628]}$ Such knowledge-driven selection of the optimal dyezeolite pair led to a material with high fluorescence efficiency and anisotropic effects, thus amenable for optical applications. ${ }^{[628]}$

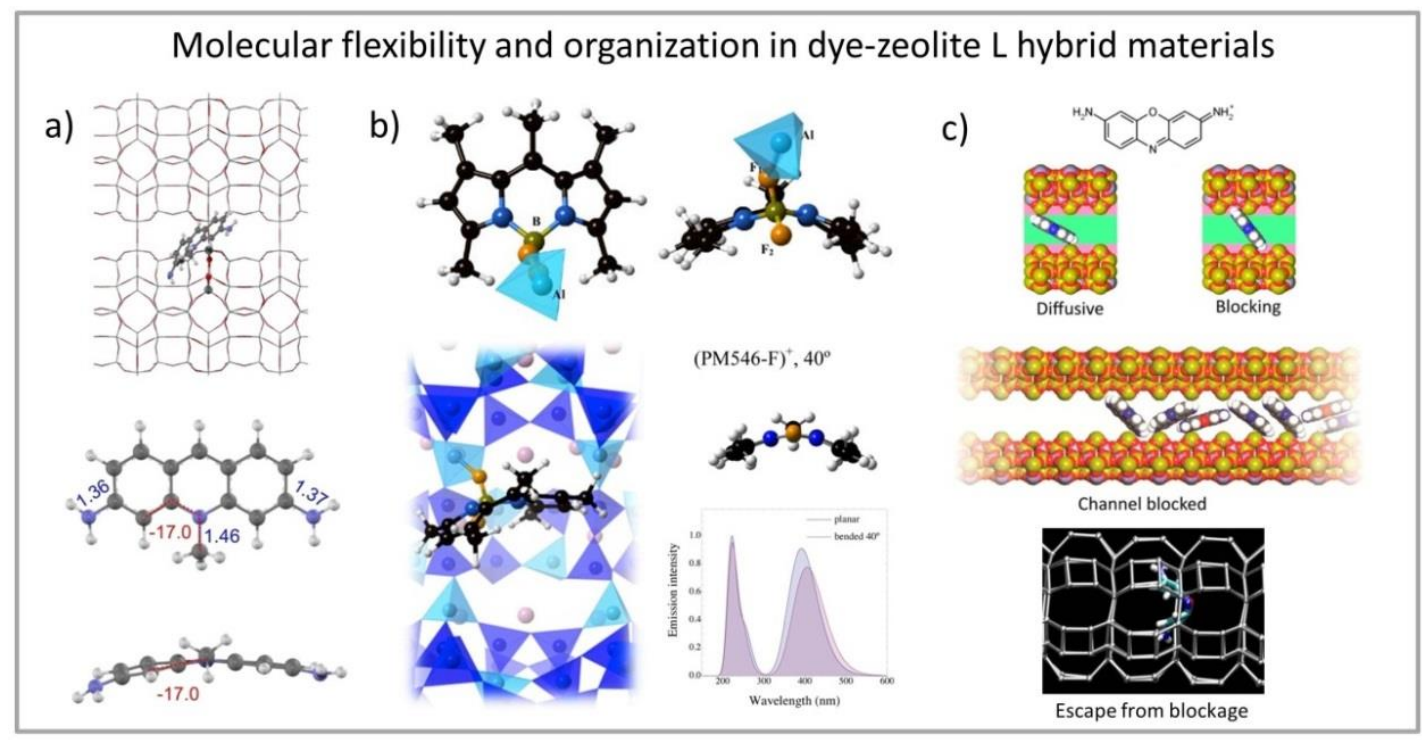

Figure 46. Confinement-induced molecular distortions: a) DFT-optimized geometry of the acriflavine molecule inside zeolite $L$ (side view along the LTL channel) and calculated geometrical parameters of the confined molecule, with distances ( $\AA$ ) and dihedral angle (degrees) in blue and red colors, respectively. [Adapted from Ref. ${ }^{[65]}$ with permission from Elsevier]. b) Configuration of a BODIPY dye in zeolite $L$ taken from a molecular dynamics (MD) simulation of the composite. The representations show the arrangement of the dye inside zeolite $L$ (viewed along the channels): a fluorine atom of BODIPY is coordinated to a zeolite Al atom. The calculated absorption spectra of the dye in the planar configuration and at the maximum bending angle are also shown. Color codes: $\mathrm{C}=\mathrm{black}, \mathrm{H}=$ white, $\mathrm{N}=$ blue, $\mathrm{B}=$ green, $\mathrm{F}=$ orange, $\mathrm{Cs}=$ pink, $\mathrm{SiO}_{4}=$ dark blue, $\mathrm{AlO}_{4}=$ light blue. [Adapted with permission from Ref. ${ }^{[696]}$ Copyright 2013 American Chemical Society]. c) Schematic illustrations of different configurations of oxonine leading either to diffusion or to blocking, and of a collective blocking mechanism in the zeolite channel, which may hinder the diffusion of the chromophore. [Adapted with permission from Ref. ${ }^{\left[{ }^{692}\right]}$ Copyright 2016 American Chemical Society]. A possible escape mechanism from the blocking configuration, implying a prominent distortion of the dye from its planar structure ${ }^{[595]}$ is shown in the black inset. [Illustration created with VMD].

Glovelike fitting was also crucial for the nonlinear-optical dye LDS 722 included in AEL: the rigid planar conformation and perfect alignment of the molecules, favored by tight confinement, allowed for a net fluorescence enhancement with respect to the AFI-dye compound, and second harmonic generation. ${ }^{[627]}$ Nonetheless, controlled formation of $\mathrm{J}$-aggregates is sometimes desirable. Recently, the strategic distribution of PIC cyanine dyes in ATS-type MgAPO-36 crystals, consisting in monomers at one edge and $\mathrm{J}$-aggregates at the other edge, enabled to realize a very efficient and one-directional antenna system. ${ }^{\text {[701] }}$

It is evident that atomistic knowledge of the supramolecular assemblies inside zeolite channels has contributed to enhance both scopes and performances of optical devices based on these composites. Such insight might help to address the complexity of the newer generations of photoactive composites, which are witnessing very fast progress. Recent examples include the synthesis of mesoporous silica hybrids using BODIPY dyes as co-templates, ${ }^{[636]}$ highly efficient FRET in an hydrophobic nanochannel-MOF incorporating fused aromatic hydrocarbons, ${ }^{[643]}$ the realization of a three-photon pumped laser in a host-guest MOF microcrystal[644] and the design of a multishelled hollow MOF (ZIF-8) in which energy transfer among included dyes and/or Pd nanoparticles is controlled by guests' positioning in the hierarchical ZIF scaffold. ${ }^{[647]}$ Understanding the link between structure and functionality in these compounds from molecular-level bases would be one of the future challenges of this area. 


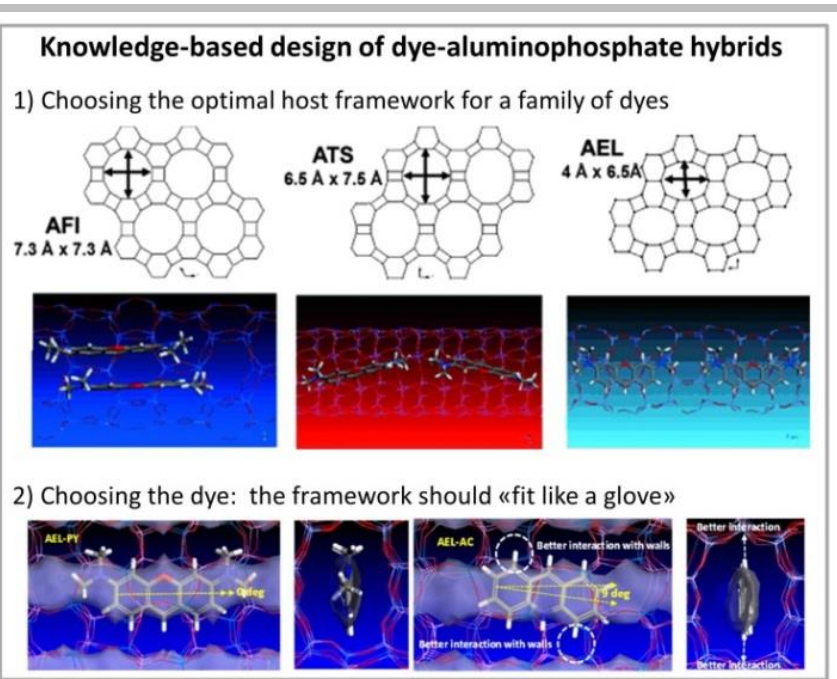

Figure 47. Optimizing organization inside porous aluminophosphates: 1) selection of the best candidate among three frameworks (AFI, ATS, AEL) for the encapsulation of pyronine dye. The pertaining optimized structures calculated from DFT are also reported, and suggest that the framework that best suits to pyronine should be AEL. [Adapted from Ref. ${ }^{[700]}$ with permission from WILEY-VCH]. 2) Choice of the dye: PY (pyronine, left) and AC (acridine, right) in the 1-D channels of AEL. The angles formed by the molecular axis with the channel axis are an estimate of the alignment of the dye: The white shaded regions indicate the free space in the channel. [Adapted with permission from Ref. ${ }^{[628]}$. Copyright 2014 American Chemical Society.]

\section{Organized materials by high pressure confinement}

The intellectual attractiveness of the realm of high pressures has captured scientists for a long time - when matter is compressed at the extremely harsh conditions of few megabars, "strange things happen" - as illustrated by a monumental review ${ }^{[702]}$ aptly entitled "The chemical imagination at work in very tight places". Earth scientists are well accustomed with the power of pressure - maybe not so harsh to strip electrons out of atoms, or turning insulators into metals, but still very high for our standards. More precisely, to study phase transitions or amorphization of minerals, geologists work "in the GPa range" - i.e., with kilobarto-megabar pressures. In this regime, unusual and fascinating facts emerge by compressing porous materials with molecules small enough to penetrate their channels. Using high or moderately high pressures, these molecules may be driven into unfavorable environments: hydrocarbons can fill hydrophilic frameworks and water hydrophobic cavities. Also, oversized or overcrowding guests can be fitted into cages or channels, and stable species might be forced to react. Most importantly for our scopes, this tremendous power, combined with the shaping effect of the matrix, can provide access to a new multitude of otherwise unfeasible confined supramolecular systems.

The intrusion of new molecules in a porous material is usually enforced using a diamond anvil cell (DAC) ${ }^{[703,704]}$ - an apparatus in which the sample is compressed hydrostatically via a pressure transmitting medium loaded into the DAC. ${ }^{[05]}$ At normal conditions, the pressure medium may be in liquid, solid, or gas state; in the latter case, it is either liquefied before loading, or directly injected at high pressure into the DAC using a gasloading device. ${ }^{[53,534,535]}$ The transmitting medium plays therefore a key role in the experiments, because it is the potential content of the pressure-created material, which should be "shaped" by the framework architecture. Care should be taken in choosing the composition of the pressure fluid because the penetration process depends on many factors other than the relative size of the host cavities and the potential guests.

A considerable number of experimental and computational evidences indicate that not all frameworks are penetrated by new species coming from the pressure-transmitting fluids namely, intrusion may not occur even if the molecules of the pressure media are small enough to pass through the pore entrances. For example, many hydrophilic frameworks, including those of natural zeolites, do not typically welcome the penetration of new species, because they already host supramolecular aggregates at room pressures. ${ }^{[128,534,706]}$ Hence, the uptake of molecules from the pressure media also depends on the species already present in the pores at room conditions, as well as various experimental variables: maximum pressure and rate of pressure increase, surface/volume ratio of the crystallites in the sample, temperature at which the experiment is performed. ${ }^{[53,534,707]}$ All these factors, together with the geometric "shaping effect" of the framework, play an important role in governing not only the pressure-driven penetration and organization process, but also its reversibility/irreversibility. Their influence should be further investigated and deeply understood in order to gain control over the process. Nevertheless, empty frameworks are generally preferable to already filled ones for fabricating organized materials with the help of high pressure. To this aim, hydrophobic zeosil frameworks - after removal of the template used in their synthesis - offer a wide platform of robust void-space architectures enabling for weak interactions with guest species: such features make them ideal candidates as "molds" for pressure-assisted supramolecular organization.

\subsection{Pressure-induced water insertion and cation exchange}

Water was the first species to be introduced through highpressure into zeolites, ${ }^{[708]}$ yet the first structural reports on pressure induced hydration or overhydration $(\mathrm{PIH})$ came many years later. ${ }^{[709,710]}$ Since then, many studies have discussed the pressure-driven injection of water inside hydrophobic ${ }^{[527,711-713]}$ or hydrophilic ${ }^{[714-717]}$ matrices, including aluminophosphates, ${ }^{[718]}$ which have advanced our understanding of water organization in tight spaces, and are systematically discussed in dedicated accounts. ${ }^{[53,128,534,535]}$

As regards hydrophobic frameworks, the forced intrusion of water was often attained using a porosimeter - an apparatus operating at pressures lower than those of DACs. ${ }^{[719]}$ This approach has also been applied to force the intrusion of electrolytic solutions. ${ }^{[719,720]}$ Such studies, nicely surveyed by a recent review, ${ }^{[719]}$ were aimed at possible energy storage applications of water intrusion/extrusion processes in porous systems $^{[721]}$ and played an important role in the development of the field, ${ }^{[119]}$ also by providing a thermodynamic description of hydrophobically confined water. ${ }^{[712]}$

Concerning hydrophilic frameworks, high-pressure overhydration studies (mostly conducted with DACs) evidenced that insertion of water molecules perturbs the pre-existing arrangement of the extraframework species, leading to the appearance of new organizational patterns and variations in the elastic properties of the framework. ${ }^{[534,722,723]}$ For instance, a recent single-crystal study on laumontite zeolite - an important constituent of the rocks forming oil reservoirs - clearly evidences the "pillar effect" played by water, i.e. the ability of counteracting the pressureinduced deformations by stiffening the framework "from the inside". ${ }^{[724]}$ Also, pressure-induced hydration of the layered zeolite mesolite showed that the different type of cations in 
alternating layers caused different spatial distribution and behavior ("structuration") of the incorporated water. ${ }^{\text {[725] }}$

The pressure-driven evolution of water supramolecular aggregates in the monodimensional channels of a synthetic gallosilicate LTL framework ${ }^{[711]}$ nicely illustrates the combined effects of high pressure and space confinement (Figure 48). At ambient pressure, the confined water molecules mainly form hydrogen-bonded clusters separated by water sheets, ${ }^{[711,726]}$ as in natural perlialite, ${ }^{[57]}$ while at higher pressures $(0.88 \mathrm{GPa})$ the water clusters and the sheets join together to form hydrogenbonded water nanotubes inside the zeolite nanochannels. By further increasing pressure, from 1.29 up to $2.64 \mathrm{GPa}$, the nanotube is gradually disrupted into isolated species interacting with the hosts. ${ }^{[711]}$ This experiment demonstrated the potential of pressure to create regular water structures inside zeolitic channels, but also that an exceedingly high compression may damage or destroy the confined supramolecular architecture.

High pressure-induced ionic exchange may be a further way to exert control over the type of guests encapsulated within hydrophilic frameworks, ${ }^{[727]}$ allowing thus to place oversized cations, like europium, ${ }^{[728]}$ in zeolites that do not normally undergo ion-exchange, like natrolite.
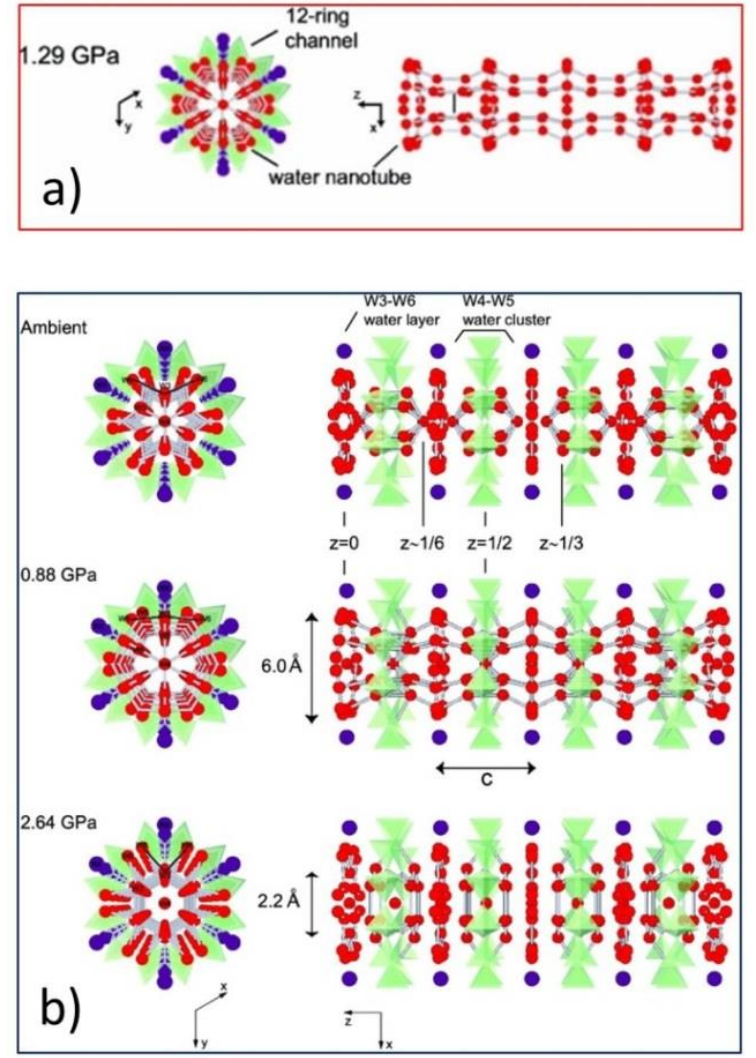

Figure 48. Water nanotubes in the 1-D channel system of an LTL-type gallosilicate. a) X-ray structure at $1.29 \mathrm{GPa}$, representing the fully-formed nanotube. b) Different water nanostructures form inside the 12-membered-ring channels of K-GaSi-LTL upon increasing pressures. Water layers and clusters, separated at ambient conditions, join together to form a tube at $0.88 \mathrm{GPa}$. At higher pressure $(2.64 \mathrm{GPa})$, the interactions of water with LTL walls become predominant: the nanotube disassembles into individual clusters. Color codes: The 12-membered ring channel windows are shown as green polyhedra, red and blue balls indicate water oxygens and $\mathrm{K}^{+}$, respectively. The grey bars between oxygen atoms represent possible hydrogen-bond interactions (distances between 2.55 and $2.9 \AA$ ). The heights of the water nanostructures along the $c$ axis are also reported. Vertical arrows indicate the size of the entrance of the water nanotubes: the access is closed at 2.64 GPa. [Adapted with permission from Ref. ${ }^{[711]}$. Copyright 2007 American Chemical Society.]
Such counterintuitive phenomenon can occur because natrolite is an auxectic material, i.e., it expands perpendicular to the stretching direction, thus causing its narrow pores to increase their volume under hydrostatic compression. ${ }^{[729]}$ When pressure is applied, the elliptical channels of natrolite are gradually converted into nearly circular ones: in this way, larger cations enter the pores and remain trapped after pressure release (Figure 49). As predicted by DFT calculations, ${ }^{[730,731]}$ ionexchange is energetically unfavorable, and must be driven by pressure - not surprisingly, the extent of Eu'll exchange increases with compression. ${ }^{[728]}$ Hence, pressure-induced ionic exchange can lead to materials where structure and composition of the original zeolite are radically altered.

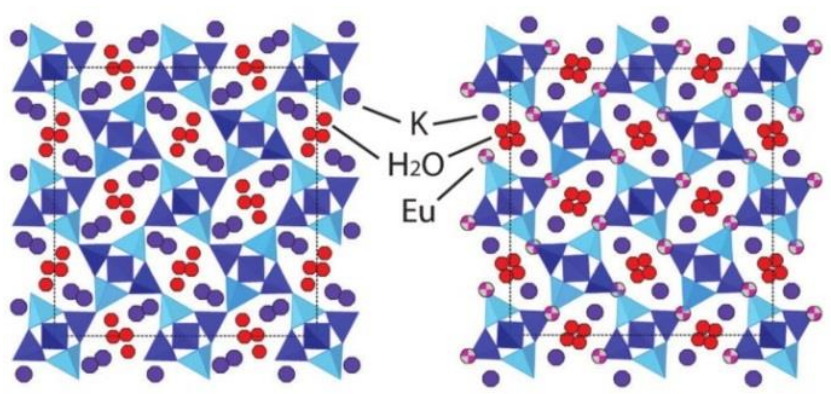

Figure 49. Pressure-driven ionic exchange. The X-ray structure of zeolite $\mathrm{K}$-natrolite is shown a) at room pressure and b) compressed at $1.5 \mathrm{GPa}$ with a $1.0 \mathrm{M} \mathrm{Eu^{ \prime \prime \prime }}$ nitrate solution as pressure-transmitting medium and cationexchange solution. The zeolite is viewed along [001], i.e. the channel axis Upon compression, europium cations (pink) are found in a site different from the potassium cations (blue balls) and water molecules (red balls) sites. High pressure also induces a significant re-organization of water and potassium cations inside the void spaces of natrolite. Dark (light) tetrahedra represent an ordered distribution of $\mathrm{Si}(\mathrm{Al})$ atoms in the framework. [Adapted from Ref. ${ }^{[728]}$ with permission from WILEY-VCH].

\subsection{Pressure-driven polymerization as a limit case of confined organization}

Pressure-assisted polymerization may be considered as a limit case of organization under space restrictions. Bulk-phase polymerization under high pressures, ${ }^{[732,733]}$ and polymerization in confined environments at ambient conditions ${ }^{[734]}$ were both known phenomena, yet their combination was documented only recently. By compressing all-silica silicalite (MFI framework type, Figure 5) in supercritical ethylene up to $1.5 \mathrm{GPa}$, and irradiating the sample with UV light, Santoro et al. ${ }^{[735]}$ reported in 2013 the formation of photo-polymerized ethylene. Optical spectroscopy and X-ray diffraction showed that the host-guest compound, after ambient pressure recovery, contained polyethylene chains and had a greater bulk modulus than silicalite due to the filling of the pores (Figure 50a). Using the same zeolite as confining matrix, the same group performed the pressure-driven-only polymerization of acetylene: compression up to $\sim 4 \mathrm{GPa}$ (without light irradiation) induced the penetration of the molecules in silicalite and their polymerization to polyacetylene chains running along the zeolite channels (Figure 50b). ${ }^{[54]}$

Now, the question arises as what happens if we use the same "mold" - the zeolite framework - and change its potential content - the pressure medium. The above experiment was thus repeated using carbon monoxide, which formed a polycarbonyl species $[-(\mathrm{C}=\mathrm{O})-]_{\mathrm{n}}$ inside silicalite. ${ }^{[736]}$ The investigators compressed mixtures of solid $\mathrm{CO}$ and silicalite in a DAC, and adopted a joint experimental-computational characterization to 
evidence the formation of the polymer: in particular, they found that the IR spectra were compatible with the structure obtained by DFT calculations. Experimental data suggested that no covalent bond was formed between the zeolite and the encapsulated polymeric moiety, while ex-situ analyses proved the irreversibility of the process. Unfortunately, as silicalite has a three-dimensional pore system (Figure 5), the $\mathrm{CO}$ molecules penetrated and polymerized into the smaller channels as well; as a consequence, the obtained polymer was not perfectly monodimensional.

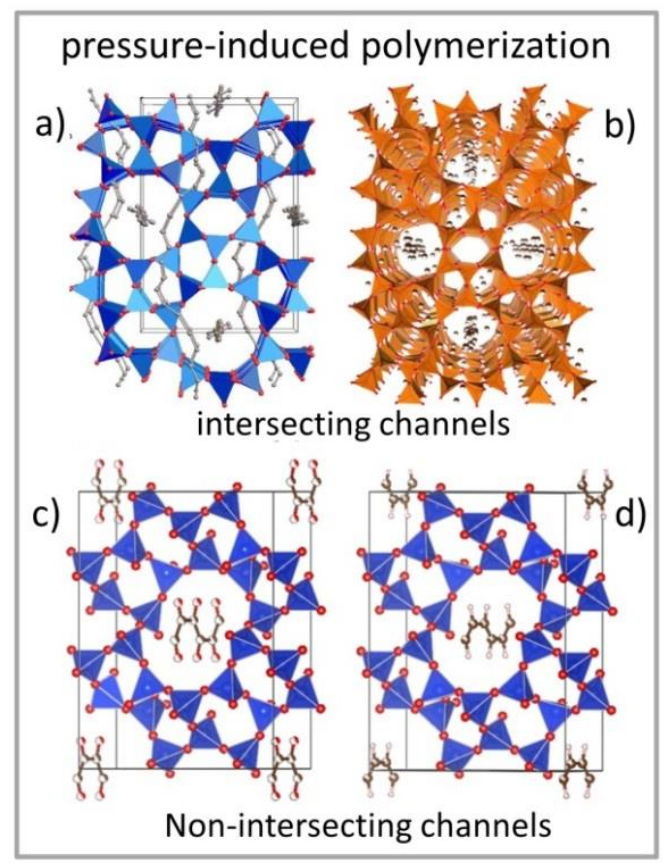

Figure 50. Pressure-fabricated confined polymers: a) strained polyethylene chains in all-silica silicalite (MFI framework type) (Color code: blue = $\mathrm{SiO}_{4}$ tetrahedra; red balls=O atoms; small grey spheres=C atoms, while $\mathrm{H}$ atoms are not shown). [Adapted by permission from Macmillan Publishers Ltd. $\left.{ }^{[75]}\right]$ b) polyacetylene in silicalite; b) polyacetylene and polycarbonyl in ZSM-22. Color codes: a) silicalite framework: $\mathrm{Si}=$ orange tetrahedra $\mathrm{O}=$ red balls. The white balls are the positions of $C$ atoms of polyacetylene from in situ high-pressure X-ray diffraction. Reprinted with permission from Ref. ${ }^{[54]}$ Copyright 2014 American Chemical Society. c,d) ZSM-22 framework: Si = blue tetrahedra; $\mathrm{O}=$ red balls. c) Polycarbonyl: grey $(\mathrm{C})$ and red $(\mathrm{O})$. d) Polyacetylene: black $(\mathrm{C})$ and white $(\mathrm{H})$. Adapted with permission from Ref. ${ }^{[737]}$ Copyright 2016 American Chemical Society.

To solve the branching problem, the researchers chose a zeolite with a mono-dimensional channel system: the all-silica ZSM-22 (TON framework type), which was compressed up to 5-10 GPa using as pressure media $\mathrm{CO}$ and acetylene, in two successive experiments. ${ }^{[737]}$ Also in this case, the applied pressure led to the irreversible formation of polycarbonyl and polyacetylene, but the use of a 1D-channel framework greatly improved the linear organization of the polymerized guests, highlighting thus in a very effective way the relevance of the purely geometrical "shaping effect" of the confining medium (Figure 50c,d).

A confining matrix with 1D-channel network -zeolite AlPO $_{4}-5$ was also recently chosen by Liu and coworkers to study the pressure behavior of confined bromine. ${ }^{[590]}$ By high pressure Raman and X-ray diffraction experiments, the researchers found that, with respect to previous studies of $\mathrm{Br}_{2}$ encapsulation, ${ }^{[586]}$ the use of high pressure led to the formation of longer bromine chains. Although a true polymerization did not occur, the distance between confined molecules gradually became comparable to the $\mathrm{Br}-\mathrm{Br}$ intramolecular bond length with increasing pressures, suggesting that the dissociation of the bromine molecules and the formation of a $\mathrm{Br}_{n}$ confined polymer should occur at even higher pressures - namely, above 24 GPa. ${ }^{[590]}$ Also iodine was found to exhibit a similar pressuredriven self-organizing behavior when confined into the same framework. ${ }^{[589]}$ Such pressure-created $\left(I_{2}\right)_{n}$ chains showed an unexpected photoluminescence behavior under compression which was absent in solid iodine, and was attributed to the unique 1D structure of the formed chains. ${ }^{[592]}$

These experiments showcase how pressure-induced polymerization is, actually, the ultimate consequence of the "hyperconfinement" regime: when confinement in nanospaces is combined with compression, intermolecular interactions may become competitive with intramolecular covalent bonds, and even dominant if the applied pressure is high enough. ${ }^{[702]}$ Also, remarkable properties may emerge simply from order enhancement, as demonstrated by the promising observation of pressure-induced photoluminescence of confined iodine chains. ${ }^{[592]}$ Nonetheless, such interesting properties acquired via compression should be maintained upon pressure release - i.e. the process should be irreversible - in order to be more easily exploitable in applications.

\subsection{Pressure-driven organization of a single type of guests}

The framework composition profoundly affects the uptake of the pressure medium in the zeolite channels, as demonstrated by several experiments. ${ }^{[706,728,738]}$ Whereas it was not possible to incorporate ethanol and methanol inside Na-mordenite - which already contained six $\mathrm{Na}^{+}$and $19 \mathrm{H}_{2} \mathrm{O}$ in the unit cell, ${ }^{[716,739]}$ Arletti et al. ${ }^{[70]}$ reported the intrusion of these molecules into the empty pores of all silica mordenite. ${ }^{[58,60]}$

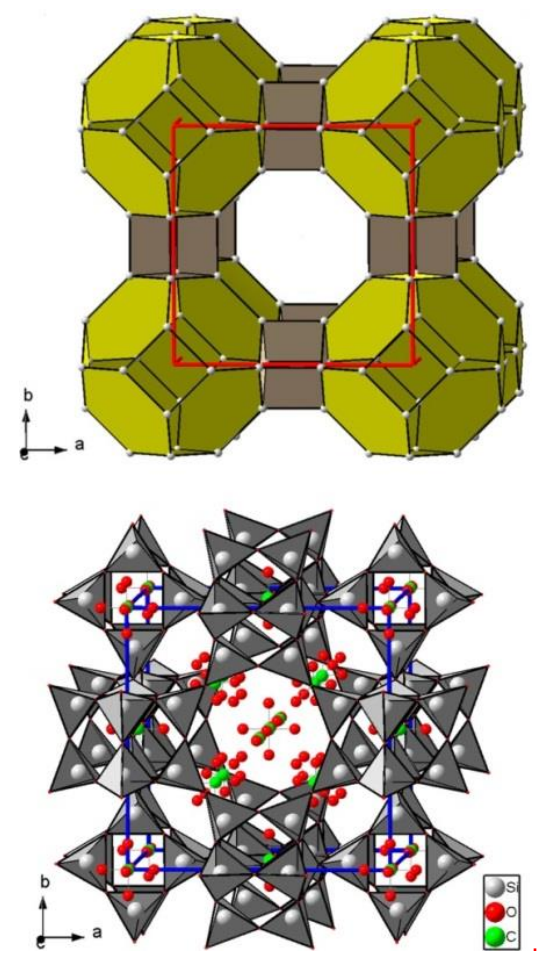

Figure 51. Pressure-induced organization of $\mathrm{CO}_{2}$ in the ITQ-29 framework a zeosil with LTA topology. (Top) The sodalite cages and the cubic cages composing the framework are colored in yellow and gray, respectively. (Bottom) The $\mathrm{CO}_{2}$-filled framework structure showing the connectivity between 
the $\mathrm{SiO}_{4}$ tetrahedral units and the location of $\mathrm{CO}_{2}$ molecules at $0.5 \mathrm{GPa}$. Gray, green, and red balls represent $\mathrm{Si}, \mathrm{C}$, and $\mathrm{O}$ atoms, respectively. [Reproduced with permission from Ref. ${ }^{[741]}$ Copyright 2017 American Chemical Society.]

These researchers also compressed all-silica mordenite by using ethylene glycol as pressure-transmitting medium. They observed that the intrusion of the glycol molecules greatly enhanced the stability of the zeolite framework against pressureinduced amorphization. Importantly, the insertion of ethylene glycol occurred already at moderate pressures - namely at 0.1 $\mathrm{GPa}$, and showed features of irreversibility upon decompression. ${ }^{[740]}$ The experiment indicated that it is not necessary to use extreme pressures to introduce organic molecules in zeolites. This finding was in line with what observed for pure water intrusion in hydrophobic ferrierite by a porosimeter ${ }^{[712]}$ and for pressure-driven encapsulation of other inorganic species, like ammonia borane. By using Raman spectroscopy, Richard et al. ${ }^{\text {[742] }}$ proved that this molecule, solid at normal conditions, could be inserted in hydrophobic silicalite (Figure 5) already at pressures of about $0.1 \mathrm{GPa}$, with a greater orientational disorder of the $-\mathrm{BH}_{3}$ and $-\mathrm{NH}_{3}$ groups compared to bulk ammonia borane crystals. X-ray diffraction analysis on the composite evidenced the formation of ammonia borane chains due to the spatial constraints, suggesting that each pore in the unit cell should contain between 2 and 3 guest molecules. ${ }^{[742]}$ Confinement and organization of simple molecules, like carbon dioxide, in three-dimensional patterns, can be also attained by using frameworks with large void spaces, like ITQ-29, ${ }^{743]}$ an allsilica analog of zeolite A (Figure 51). Santamaria-Perez et al. studied the behavior of this system up to $20 \mathrm{GPa}$, establishing that $\mathrm{CO}_{2}$ intrusion at pressures below $0.5 \mathrm{GPa}$ stiffened the structure preventing the amorphization of this large-pore zeolite. ${ }^{[741]}$ Notably, the intruded fluid is actually tailored by the void-space-network: among the 13 encapsulated molecules per unit cell, twelve occupy the large $\alpha$-cage and only one the small sodalite $\beta$-cages. The filling of the $\alpha$-cage by the $\mathrm{CO}_{2}$-assembly is presumably the reason why this framework did not collapse under the extreme pressures used in the experiment. ${ }^{[741]}$

\subsection{High pressure as driving force for supramolecular organization of multiple species}

All previous examples considered the intrusion of a single type of species in hosts with 1D-, 2D, or 3D channel systems.

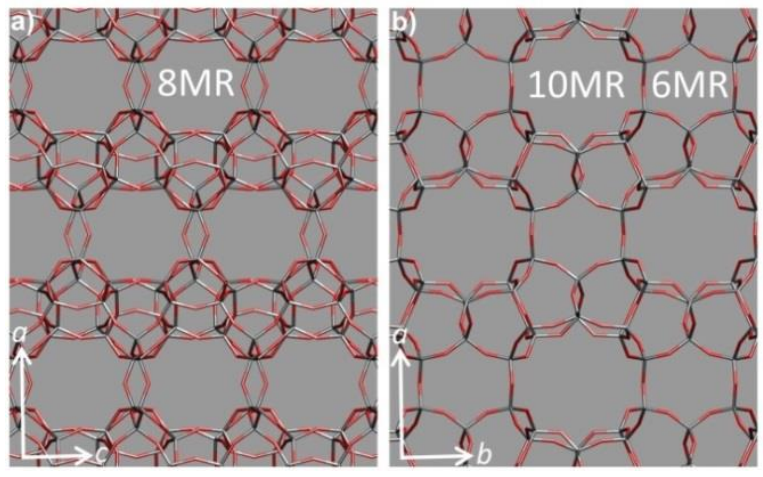

Figure 52. Ferrierite (FER framework type) has a two-dimensional system of intersecting channels, into which molecular species may be organized in 2D patterns. The image shows two views of the FER framework a) in the ac plane, showing the eight-membered ring channels, b) in the ab plane, showing the ten- and six-membered ring channels ( $\mathrm{O}=\mathrm{Red}$ sticks; $\mathrm{Si}=$ grey sticks). [Adapted from Ref. ${ }^{[55]}$ with permission from WILEY-VCH]
The complexity of the organization problem drastically increases when the species to be confined have different chemical nature, i.e. if the pressure medium is a mixture.

This is, for example, the case of an electrolytic $\mathrm{MgCl}_{2} \cdot 21 \mathrm{H}_{2} \mathrm{O}$ solution injected by Arletti et al in ferrierite. ${ }^{[44]}$ This zeosil has a 2D-system of intersecting channels: eight-membered ring channels running in the $y$ direction cross two parallel channel systems of different diameter, defined by six- and tenmembered rings, running in the $z$ direction (Figure 52). The sixmembered ring cavities are also named ferrierite cages. ${ }^{[745]}$
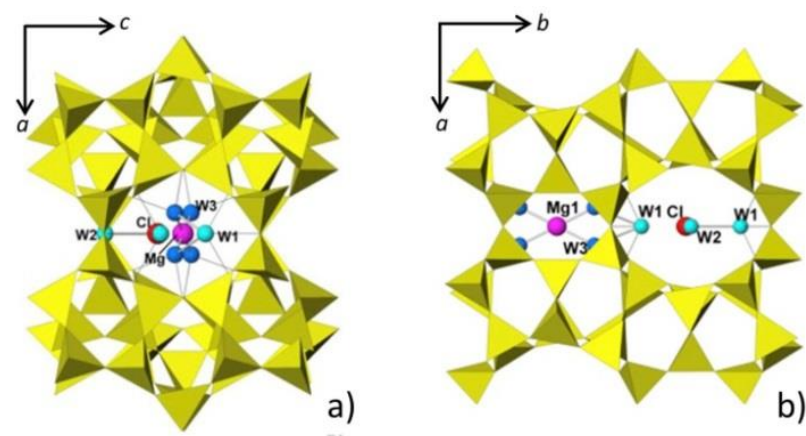

Figure 53. Organization of $\mathrm{Mg}, \mathrm{Cl}$, and $\mathrm{H}_{2} \mathrm{O}$ in the $2 \mathrm{D}$ channel system of ferrierite. Both ions and water molecules in the $\mathrm{MgCl}_{2}$ aqueous solution used as pressure media are intruded in ferrierite: $\mathrm{Mg}$ in the 6-membered ring cage and $\mathrm{Cl}$ in the 10-membered ring channels. Atom positions are obtained from refinement of the data collected at $0.28 \mathrm{GPa}$. FER framework is represented as yellow tetrahedra. [Adapted from Ref. ${ }^{[744]}$ with permission from Elsevier].

Aim of the investigators was to ascertain whether both ions and water molecule could penetrate in ferrierite. Indeed, the insertion took place at relatively low pressures (below $0.2 \mathrm{GPa}$ ) and was reversible, as demonstrated by diffraction experiments. The refinement showed that all the components of the pressure fluid penetrated in the zeolite. A two-dimensional pattern emerged, featuring $\mathrm{Mg}$ at the center of the ferrierite cage, surrounded by four water molecules in a square planar symmetry, and chlorine in the ten membered ring channels, interacting with two water molecules (Figure 53). One remarkable finding was that the location of the $\mathrm{Mg}$ cation perfectly matched that observed by Alberti et al. on a natural ferrierite sample from Sardinia, in which Mg cations coordinated six water oxygens in a octahedral geometry. ${ }^{[76]}$ Despite the different nature of the extraframework species, the identical placement of the bivalent cation in the mineral and in the pressure-created material underlines how the realization of new supramolecular patterns by high-pressure confinement could benefit from the knowledge of supramolecular organization of water and cations in natural zeolites.

Water-alcohol mixtures of different composition are often used as pressure transmitting media. Mixtures of methanol:ethanol:water in proportions 16:3:1 are commonly adopted, and were also used in two high-pressure investigations ${ }^{[707,747]}$ on siliceous ferrierite. This confining matrix, according to experimental/computational studies, ${ }^{[748-750]}$ should be one of the most selective frameworks for the separation of water/alcohol mixtures - an important step in bioethanol production. ${ }^{[751]}$ Indeed, separating molecules of similar size has always been a challenge, especially if they form hydrogen bond networks - like e.g., in water/methanol/ethanol mixtures. In both high-pressure studies on Si-ferrierite, however, only water molecules penetrated into the framework, ${ }^{[707,747]}$ 
already at moderate pressure values $(0.2 \mathrm{GPa})$ - similar to what observed with the intrusion of pure water in the same zeolite. ${ }^{[712]}$

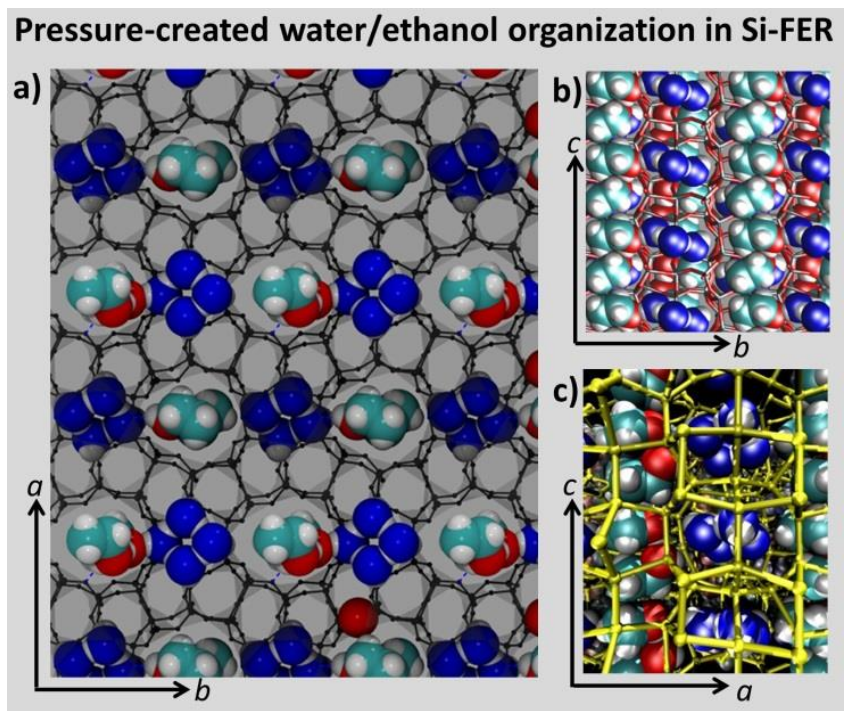

Figure 54. The two dimensional pattern of water and ethanol obtained by compressing Si-FER with a water-ethanol mixture up to $1.3 \mathrm{GPa}$. The three different views highlight, in particular: a) the water tetrameric squares; b) the chains of ethanol dimers; c) the confinement of the supramolecular architecture inside the zeolite. This pattern is maintained after recovery to ambient pressure, showing how pressure-enhanced confinement in nanospaces ("hyperconfinement") can turn liquid mixtures of simple molecules into organized materials. (Water=blue and white; ethanol=red, cyan and white FER framework: a) grey sticks and tetrahedra; b) red-gray sticks, c) yellow ball-and-sticks.) [b): Adapted from Ref. ${ }^{[55]}$ with permission from WILEY-VCH].

Let us see what happens if we use the same framework and change the composition of the pressure medium by increasing the water content. Arletti et al. ${ }^{[55,752]}$ compressed hydrophobic ferrierite ${ }^{[58]}$ in an ethanol:water $=1: 3$ mixture up to $1.2 \mathrm{GPa}$ and detected penetration of the fluid already at relatively low pressures. X-ray powder diffraction data combined with firstprinciples modelling showed that the ferrierite framework had separated the ethanol-water mixture into nearly isolated ethanol dimer wires and water tetrameric squares, forming a confined two-dimensional pattern of alternating supramolecular units (Figure 54), which remained stable even upon recovery to normal conditions. ${ }^{[55]}$ Geometric analyses of these units and of the ferrierite cages indicated that the 6- and 10-membered rings channels have the right size and shape to host, respectively, the water tetramers and the ethanol dimers. Hence, the shaping effect of the ferrierite framework plays a key role in the organization of water and ethanol.

In general, the irreversibility of pressure-induced transformations is relevant for potential applications of materials obtained in this way. Although a moderate compression can be sufficient to achieve penetration, ${ }^{[712,747]}$ higher pressures might be needed for irreversibile organization. The inherent preference of water molecules to congregate together, both in hydrogen-bonded liquid mixtures ${ }^{[753]}$ and under confinement (as discussed in this review), is likely one of the main factors responsible of the separation of water and ethanol in supramolecular blocks perfectly tailored by the void spaces of ferrierite.

\subsection{High pressure and MOFs}

The mechanical stability of MOFs, generally lower than zeolites, may represent a big practical problem for certain applications. Nonetheless, by virtue of the immense structural versatility of these materials, MOF frameworks might actually be optimized to improve stiffness. Of course this task would be easier if we knew how they respond to harsh pressure conditions. Although MOFs are still relatively little explored at high-pressure, some examples of robust frameworks, like ZIF-8 ${ }^{[610]}$ and ammonium metal formates ${ }^{[754]}$ are already known, and their compression behavior can be studied by in situ diffraction. ${ }^{[755]}$ By analyzing MOFs response to pressures which can push their resistance to the limit we could get precious information for the design of more robust frameworks with potential applications in high-pressure processes. A recent example is the "retrofitting" of MOF-520 (that amorphizes at $2.81 \mathrm{GPa}$ ) with an extra bridging ligand, which allows the retrofitted material to remain crystalline up to $5.5 \mathrm{GPa}$ (Figure 55)..$^{756]}$ Moreover, pressure-driven selectivities, e.g for $\mathrm{Ne} v s$. Ar incorporation in metal formates, ${ }^{[754]}$ and unique mechanical effects, like reversible amorphization ${ }^{[757]}$ or negative linear compressibility ${ }^{[758]}$ have been uncovered. A recent study highlighted an intriguing pressure-induced effect named "zone collapsing", i.e. the amorphization of limited regions of the sample, leading to injection of guests from collapsing pores into the still nearly intact regions of the MOF, thus preventing their amorphization. ${ }^{\text {[759] }}$
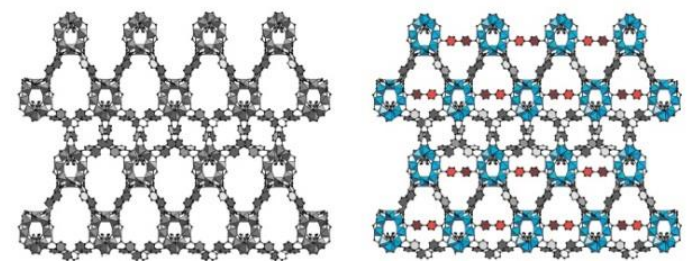

Figure 55. MOF retrofitting to withstand compression: The original MOF520 (in gray, left) was "retrofitted" by an extra 4,4'-biphenyldicarboxylate ligand, which acts as a "girder" improving mechanical stability (MOF-520-BPDC, right). [Reprinted with permission. ${ }^{[756]}$ Copyright 2017 American Chemical Society.]

Molecular inclusion in MOF's spaces can be obtained also at lower pressures, as shown recently by $\mathrm{Im}$ et al, ${ }^{[760]}$ that, at 0.3 $\mathrm{GPa}$, selectively replaced, in the as-synthesized hydrophobic MOF MIL-47(V), the terephthalic acid (TPA) with methanol using a methanol/ethanol/water mixture as pressure transmitting fluid (see Figure 56, left). The system was then compressed up to $1.91 \mathrm{GPa}$, and, upon pressure release, methanol remained into the 1D-channels of this MOF. ${ }^{[760]}$ Instead, by compressing the MOF with water, the exchange occurred at higher pressures $(1.0 \mathrm{GPa})$ and water incorporation was reversible (Figure 56, right). Such a different behavior was attributed to the hydrophobic nature of the framework: actually, the exchange of TPA with methanol occurs also at ambient pressure simply by pouring methanol solution into the MOF. ${ }^{[760]}$ This study evidenced that the pressure-induced incorporation depends strongly on the molecules in the pressure medium, in line with what found in many experiments on zeolites, as previously discussed. Also note that the starting system (the assynthesized MOF) contains already a supramolecular assembly, formed by the TPA molecules. Such a system is readily disassembled upon pressure-intrusion of methanol molecules, which self-assemble into a new confined architecture (Figure 56). 


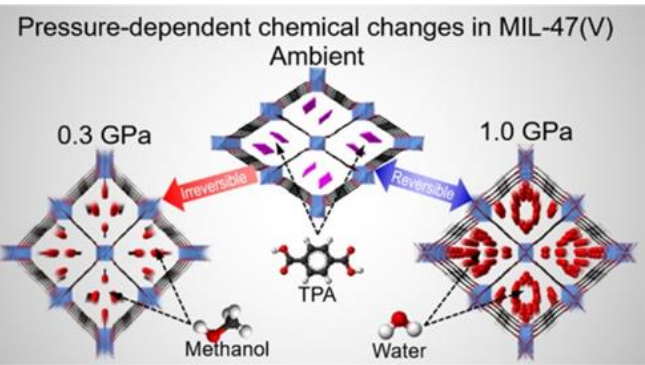

Figure 56. Pressure-induced guest exchange: At moderate pressures, in the 1D channel MOF MIL-47(V), (at the center) terephthalic acid (TPA) can be exchanged with methanol molecules (left), which are ordered with either the $C$ or $\mathrm{O}$ atoms toward the $\mathrm{VO}_{6}$ octahedral nodes of the MOF (blue). Much higher pressures are needed to induce water intrusion into hydrophobic MIL-47(V) framework (right). Color cores: $\mathrm{O}=$ green, $\mathrm{C}=$ grey, $\mathrm{H}=$ white. [Reproduced with permission from Ref. ${ }^{[760]}$ Copyright 2016 American Chemical Society.]

To gather insight into such processes, the contribution of modeling studies, especially when combined with experiments, could be invaluable. ${ }^{[761-764]}$ Methodologies for determining elastic constants and other strain-related properties ${ }^{[765]}$ at a high level of accuracy have been implemented ${ }^{[766,767]}$ - and applied in order to improve our understanding of the mechanical response and stability of this topical family of materials. ${ }^{[175]}$ Tan et al, for example, used $a b$ initio periodic density functional theory to study the elastic behavior of two nanoporous zeolite-imidazolate frameworks with the same chemical composition but different topological features, thus highlighting important relationships between their differences in structure and elastic properties. ${ }^{[768]}$ In a subsequent study, Ryder et al investigated theoretically the bulk modulus of a class of zirconium MOFs in order to assess the mechanical resistance of the framework against the imposed hydrostatic pressure, thus determining the average mechanical

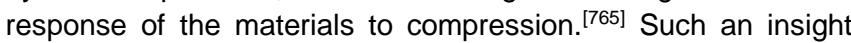
will become even more important in the future, particularly in designing new applications of these materials and stimulating further development of this vibrant research area that has contributed to change "our perception of the solid state". ${ }^{769]}$

\section{Conclusion and perspectives}

The organizing behavior of molecules, ions or nanoparticles in regular porous matrices was the subject of this work, which was aimed to show how the organization of guest species under nanospace confinement is a multifaceted and complex problem. From tight clathrate cages, to zeolite channel architectures, up to the tunable mesopores of organosilicas or MOFs, organization under confinement is not simply a matter of shape, size and geometry. Geometry - in particular the size-shape matching of host and guest - certainly helps in selecting species to be incorporated in a given host, and vice-versa. However, small molecules can form organized patterns even within oversized cages, provided that there is a further stimulus - for example, specific host-guest interactions; an external pressure or field; presence of other species (e.g. solvent or template molecules) - that imparts additional confinement. Conversely, molecules can be introduced into undersized spaces thanks to the structural flexibility of both host and guest. This factor strongly influences and often determines how organization is achieved and maintained, destroyed or converted - especially under tight space restrictions. Organization is in itself a dynamic process.
The time variable is crucial in spatially-restricted (re)organization processes: efforts should be directed towards further understanding how organized assemblies dynamically form, and evolve in time - which is particularly important for host materials characterized by high framework flexibility.

A prominent common feature of the hosts is the presence of an interface with the guest molecules: the inner pore surface. Though weak in some cases, interactions of the incorporated species with the interface - van der Waals, electrostatic, hydrogen/halogen bonding, or coordination - finely influence the dynamics and response properties of the confined aggregates. Moreover, guest species may interact significantly with point defects of the pore surface.

Obviously, all those system have in common also the interface with the rest-of-the-world: the external surface, and particularly pore entrances. The entrance of guests in confined spaces is often the first step of their assembling processes. Functionalization of pore entrances offers the opportunity to control the organization process and then to transfer hierarchically the organization to the macroscale.

All confined assemblies, irrespective of the matrix, are stimuli responsive. Useful information on their stability and their dynamics can be obtained by studying their changes upon application of a stimulus - e.g. electromagnetic, thermal, and mechanical. This work focused on the latter one, because the combination of high pressure and regular porous matrices has emerged as an appealing route to confined supramolecular organization. ${ }^{[55]}$ In general, although the molecular-level bases of high-pressure phenomena are largely unexplored, the perspectives appear particularly exciting. New host types have also been created: for example, starting from an economically convenient precursor, a new zeolite has been obtained by high pressure treatment, showing significantly better catalytic performances than the parent material. ${ }^{[770]}$

Overall, striving to dissect the subtle mechanisms of molecular organization would be instrumental in achieving a better contro over them. This is suggested, for example, by the increase in scope of several applications - luminescent clusters, quantum dot arrays, or antenna systems - initially devised in zeolites and then implemented in MOFs or mesoporous materials: ${ }^{[378,646,771]}$ such extension has surely benefitted, directly or indirectly, from the expertise acquired with zeolites. A profound knowledge of the host-guest interactions would facilitate the realization of predesigned confined molecular superstructures to be used, ultimately, as active components of functional devices. In this sense, multitechnique studies of the organization of guest species in natural zeolites, clathrate hydrates and other minerals at different pressure/temperature conditions will greatly enhance the fundamental understanding of the interactions that govern, for example, mechanical stability. In this sense, "paleoinspiration "[772] - is gaining increasing momentum in the scientific community. The idea is to transfer to modern materials the environmental resilience and other interesting properties of ancient materials, like Roman cements or the Blue Maya pigment, typically based on minerals. Indeed inclusion compounds of engineered clays ${ }^{[47]}$ are beginning to show actual potential as eco-friendly functional materials. ${ }^{[773-778]}$ Such progress was also fostered by modeling studies - that suggested, for example, to contrast the worsening in the mechanical properties of a clay (caused by the incorporation of dyes) via the co-adsorption of surfactants in the interlayer space of the clay. ${ }^{[779-781]}$ Taking inspiration from Blue Maya, the indigo molecule was recently encapsulated into zeolite $L$ microcrystals yielding an exceptionally robust colorant: ${ }^{[782]}$ the insight from 
simulations ${ }^{[191]}$ was instrumental for the successful blockage of the crystal terminations. An atomistic knowledge of natural host matrices is also important for their use in environmental protection (e.g., absorption of organic pollutants by layered hydroxides, ${ }^{[783,784]}$ sequestration of trace uranium in multiphase iron minerals ${ }^{[785]}$ ) and in the realization of new materials via sustainable fabrication approaches; for instance, using a vaporinduced approach the direct synthesis of zeolites starting from clay minerals has been recently performed. ${ }^{7866]}$

As regards the concrete use of these composites in optoelectronic applications, the distribution of the guests inside the porous matrix, and the related changes in the photophysical properties remain major issues, nearly for all the considered cases. In the endeavor to attain a more uniform distribution of the luminescent centers, an accurate characterization plays a key role. Though obviously limited to luminescent guests or probes, single-molecule optical spectroscopy techniques are perhaps best suited to address the microscopic characterization of hybrid complexes - especially when combined with other experimental techniques (e.g. NMR, Raman) or computational modeling. These approaches are unvaluable in probing pore structure and diffusion dynamics in mesoporous systems as well as the optical anisotropy in zeolite host-guest compounds.

The appealing luminescent properties of zeolite-encapsulated metal clusters have been disclosed only relatively recently. The latest advances, concerning particularly Ag-zeolites, raised great expectations for their applications in photonics and as phosphors in lighting, which are showcased in two very recent reviews. ${ }^{[43,445]}$ Further amelioration efforts are however necessary for their actual use and commercialization e.g. as LED phosphors. For example, the emission and excitation/absorption properties of rare-earth or organic-based phosphors appear still superior, as the Ag zeolites are normally activated with wavelengths below $375 \mathrm{~nm}$ and a wider excitation range would be preferable. On the bright side, besides outstanding luminescence, they display comparable quantum efficiencies, thermal behavior and also high stability. Actually the zeolite confinement is very effective as a protective environment, but the composites still remain sensitive to humidity, that alters their photophysical properties. Viable solutions may involve the embedding of $\mathrm{Ag}$-loaded zeolites in polymeric matrices, or the sealing of the zeolite channel crystal terminations with functionalized stopcocks. ${ }^{[616]}$

The shortcomings of the uneven distribution of clusters in the crystalline porous matrix are somewhat mitigated in catalysis and sensing uses. For example high stability combined with the extraordinary luminescence properties of silver clusters in zeolite microcrystals are appealing for applications such as bio imaging and bio sensing. ${ }^{[787]}$ Also, recent promising attempts of realizing greatly effective environmental sensors make use of crystalline zeolite nanoparticles in the fabrication of photonic structures. ${ }^{[788]}$ Zeolite L-based antenna materials guarantee a maximum loading of the donor molecules - which results in an extremely high speed for exciton transport - and a low loading of acceptor molecules, thus minimal self-absorption losses: the acceptors can nearly quantitatively capture the excitation energy. ${ }^{[665]}$ Though the composites are technically suitable for use in solar cells and sensing applications (Chapter 7), acidity inside the zeolite $\mathrm{L}$ channel is still a limiting factor towards attaining maximum concentration of donor molecules inside the host. ${ }^{[618]}$ Nevertheless, these composites have proven to be thermally robust, ${ }^{[133]}$ and mechanically resilient up to GPa pressures;; ${ }^{[64]}$ in addition, the host matrix is easy to synthesize in form of disc- shaped crystals, for which a uniform filling of dye molecules along the channels can be accomplished. ${ }^{789]}$

Besides mesoporous systems and MOFs, nitrido-[258] or chalcogenide open frameworks appear particularly appealing as alternative host systems for potential photophysical applications because of their electronic properties - e.g. semiconductivity which allow hosts to take part in energy transfer[269] or photocatalytic ${ }^{[790]}$ processes. They still remain, however, more difficult to synthesize compared to zeolites.

One aim of this review was to highlight the usefulness of computational models in connecting macroscopic properties of functional host-guest compounds with their molecular level structure and behavior. An important advantage of modeling is that it is possible to dissect the complexity of the real system and evaluate the separate effects of different variables (composition, temperature, concentration, etc.) on a macroscopic property of the system. However, although theoretical studies provide insight and have predictive ability, ${ }^{[171]}$ it is still not possible for computational models to reproduce the complexity of the real material. Though in different manner, all of the systems presented herein show great complexity: the number and variety of guest species, their distribution in the pores, the structural defects of the porous matrix, the effects of temperature on the guests' dynamics and framework flexibility. The experimental data (e.g., the photophysical response of the material) depend on these variables in very complex manners. Experimental and computational scientist should endeavor to design together and jointly adopt integrated strategies to set-up increasingly realistic model systems. Similar approaches for the elucidation of molecule-to-material conversion processes have made progress thanks to the combined endeavors of experimentalists and computational researchers. ${ }^{[791]}$ As also evidenced by the presented examples, the information obtained by molecular simulation has already contributed to design hostguest materials with improved optical properties. ${ }^{[28]}$ The integration between experiment and theory is particularly advanced in clathrate hydrate research, ${ }^{[315]}$ favouring progress also in the emerging field of confined hydrates. ${ }^{[346]}$ Calculations have become important to characterize disordered systems such as zeolite-metal clusters compounds, and the progress of multiscale modeling ${ }^{[792]}$ has helped to broadening length and time scales in simulation. Nevertheless, the effort should be systematic to bridge the still existing gap between the need of a quantum mechanically consistent description and the complexity of the actual system. A truly combined experimental-theoretical approach would be based ideally on the joint simplification of the experimental system and augmentation of the computational model. In this sense, the availability of nearly defect-free crystals or the use of lower-dimensionality systems, for example 2Dzeolites, would be particularly helpful for a joint characterization. The understanding thus gathered, fueled by curiosity, ${ }^{[793]}$ could foster substantial improvements of current fabrication/ characterization techniques, and help envisioning new directions for the design and practical application of these materials. 
I am a Computational Chemist and I teach Physical Chemistry at the Insubria University in Como, Italy. My research activity includes the simulation of molecules, materials, and interfaces at both normal and high pressure/high temperature conditions. I find porous materials and their guests especially challenging and captivating.

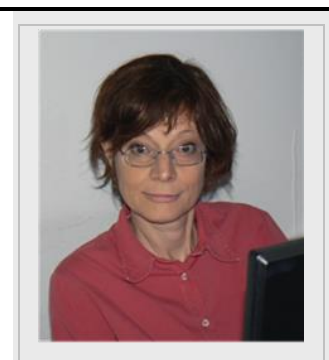

[23]

\section{Acknowledgements}

I gratefully thank the ChemPhysChem's Editorial Team and particularly the EIC Dr. Greta Heydenrych for inviting me to contribute this review. Rossella Arletti, Gion Calzaferri, Salvatore Coluccia, Ettore Fois, Aldo Gamba, Diego Gatta, Gianmario Martra, Simona Quartieri, Giovanna Vezzalini are acknowledged for the work done together and for the excellent discussions that inspired this work in many different ways. I gratefully thank the anonymous referees of this paper for their highly valuable comments and suggestions. Andrea Stangoni is credited for the frontispiece illustration. Italian MIUR projects ImPACT (FIRB RBFR12CLQD), Zapping (PRIN 2015HK93L7) and Uninsubria FAR2017 are acknowledged for funding.

Keywords: clathrates $\bullet$ host-guest interactions $\bullet$ zeolites $•$ mesoporous materials $\cdot$ metal-organic framework

[1] J.-M. Lehn, Supramolecular Chemistry, Wiley-VCH Verlag GmbH \& Co. KGaA, Weinheim, FRG, 1995

[2] J.-M. Lehn, Angew. Chem. Int. Ed. Engl. 1988, 27, 89-112; Angew. Chem. 1988, 100, 91-116.

[3] G. M. Whitesides, J. P. Mathias, C. T. Seto, Science 1991, 254, 1312-1319.

[4] V. Balzani, A. Credi, F. M. Raymo, J. F. Stoddart, Angew. Chem. Int. Ed. 2000, 39, 3348-3391; Angew. Chem. 2000, 112, 3484-3530.

[5] P. Ceroni, A. Credi, M. Venturi, Chem. Soc. Rev. 2014, 43, 40684083.

[6] S. Kassem, T. van Leeuwen, A. S. Lubbe, M. R. Wilson, B. L. Feringa, D. A. Leigh, Chem. Soc. Rev. 2017, 46, 2592-2621.

[7] J.-M. Lehn, Angew. Chem. Int. Ed. 2013, 52, 2836-2850; Angew. Chem. 2013, 125, 2906-2921.

[8] D. Philp, J. F. Stoddart, Angew. Chem. Int. Ed. Engl. 1996, 35 1154-1196; Angew. Chem. 1996, 108, 1242-1286.

[9] C. Cheng, J. F. Stoddart, ChemPhysChem 2016, 17, 1780-1793.

[10] R. D. Astumian, S. Mukherjee, A. Warshel, ChemPhysChem 2016 17, 1719-1741.

[11] C. Pezzato, C. Cheng, J. F. Stoddart, R. D. Astumian, Chem. Soc. Rev. 2017, 46, 5491-5507.

[12] V. Balzani, A. Credi, M. Venturi, ChemSusChem 2008, 1, 26-58.

[13] G. M. Whitesides, B. Grzybowski, Science 2002, 295, 2418-2421.

[14] G. A. Ozin, A. Kuperman, A. Stein, Angew. Chem. Int. Ed. Engl. 1989, 28, 359-376; Angew. Chem. 1989, 101, 373-390.

[15] L. V. Interrante, M. J. Hampden-Smith, Chemistry of Advanced Materials : An Overview, Wiley-VCH, 1998.
[16]

[17]

[18]

[19]

[20]

[21]

[22]

[24]

[25]

G. A. Ozin, Adv. Mater. 1992, 4, 612-649.

M. E. Davis, Nature 2002, 417, 813-821.

E. G. Derouane, J. C. Védrine, R. Ramos Pinto, P. M. Borges, L. Costa, M. A. N. D. A. Lemos, F. Lemos, F. Ramôa Ribeiro, Catal. Rev. Sci. Eng. 2013, 55, 454-515.

J. Weitkamp, Solid State Ionics 2000, 131, 175-188.

A. Corma, Chem. Rev. 1997, 97, 2373-2419.

A. Corma, Angew. Chem. Int. Ed. 2016, 55, 6112-6113; Angew. Chem. 2016, 128, 6218-6219.

B. M. Weckhuysen, S. Kitagawa, M. Tsapatsis, ChemPhysChem 2018, 19, 339-340 and Special Issue: Reactions in Confined Spaces: ChemPhysChem 2018, 19 (4), 335-546.

N. J. Turro, Acc. Chem. Res. 2000, 33, 637-646.

J. C. Scaiano, H. García, Acc. Chem. Res. 1999, 32, 783-793.

S. Hashimoto, J. Photochem. Photobiol. C 2003, 4, 19-49.

[26] V. Ramamurthy, Y. Inoue, Supramolecular Photochemistry: Controlling Photochemical Processes, Wiley, 2011.

[27] V. Ramamurthy, P. Lakshminarasimhan, C. P. Grey, L. J. Johnston, Chem. Commun. 1998, 2411-2424.

[28] N. Alarcos, B. Cohen, M. Ziółek, A. Douhal, Chem. Rev. 2017, 117, 13639-13720.

[29] G. Calzaferri, S. Huber, H. Maas, C. Minkowski, Angew. Chem. Int. Ed. 2003, 42, 3732-3758; Angew. Chem. 2003, 115, 3860-3888.

[30] I. Stassen, N. Burtch, A. Talin, P. Falcaro, M. Allendorf, R. Ameloot, Chem. Soc. Rev. 2017, 46, 3185-3241.

[31] D. D. Medina, T. Sick, T. Bein, Adv. Energy Mater. 2017, 7, 1700387.

[32] R. M. Barrer, in Non-Stoichiometric Compd. (Ed.: L. Mandelcorn), Academic, New York, 1964, pp. 309-437.

[33] D. A. Keen, A. L. Goodwin, Nature 2015, 521, 303-309.

[34] T. Förster, Ann. Phys. 1948, 248, 55-75.

[35] M. M. Yatskou, M. Meyer, S. Huber, M. Pfenniger, G. Calzaferri, ChemPhysChem 2003, 4, 567-587.

[36] G. Calzaferri, Langmuir 2012, 28, 6216-6231.

[37] H. S. Kim, K. B. Yoon, Coord. Chem. Rev. 2014, 263-264, 239-256.

[38] L. Maggini, D. Bonifazi, Chem. Soc. Rev. 2012, 41, 211-241.

[39] C. Leiggener, G. Calzaferri, ChemPhysChem 2004, 5, 1593-1596.

[40] L. Gartzia-Rivero, J. Bañuelos, I. López-Arbeloa, Materials 2017, 10, 495.

[41] T. Sun, K. Seff, Chem. Rev. 1994, 94, 857-870.

[42] R. Seifert, A. Kunzmann, G. Calzaferri, Angew. Chem. Int. Ed. 1998, 37, 1521-1524; Angew. Chem. 1998, 110,1603-1606.

[43] E. Coutiño-Gonzalez, W. Baekelant, J. A. Steele, C. W. Kim, M. B. J. Roeffaers, J. Hofkens, Acc. Chem. Res. 2017, 50, 2353-2361.

[44] P. Demontis, G. B. Suffritti, Chem. Rev. 1997, 97, 2845-2878.

[45] D. Bougeard, K. S. Smirnov, Phys. Chem. Chem. Phys. 2007, 9 , 226-245.

[46] T. C. T. Pham, S. Docao, I. C. Hwang, M. K. Song, D. Y. Choi, D. Moon, P. Oleynikov, K. B. Yoon, Energy Environ. Sci. 2016, 9 , 1050-1062.

[47] G. Schulz-Ekloff, D. Wöhrle, B. van Duffel, R. A. Schoonheydt, Microporous Mesoporous Mater. 2002, 51, 91-138.

[48] J. Caro, F. Marlow, M. Wübbenhorst, Adv. Mater. 1994, 6, 413-416

[49] G. Calzaferri, K. Lutkouskaya, Photochem. Photobiol. Sci. 2008, 7, 879-910.

[50] K. V. Rao, A. Jain, S. J. George, J. Mater. Chem. C 2014, 2, 30553064.

[51] Y. Wang, H. Li, CrystEngComm 2014, 16, 9764-9778.

[52] A. Devaux, F. Cucinotta, S. Kehr, L. De Cola, in Funct. Supramol. Archit., WILEY-VCH Verlag \& Co. KGaA, Weinheim, Germany, 
2014, pp. 281-342.

[53] G. D. Gatta, Y. Lee, Mineral. Mag. 2014, 78, 267-291.

[54] D. Scelta, M. Ceppatelli, M. Santoro, R. Bini, F. A. Gorelli, A. Perucchi, M. Mezouar, A. Van Der Lee, J. Haines, Chem. Mater. 2014, 26, 2249-2255.

[55] R. Arletti, E. Fois, L. Gigli, G. Vezzalini, S. Quartieri, G. Tabacchi, Angew. Chem. Int. Ed. 2017, 56, 2105-2109; Angew. Chem. 2017, 129, 2137-2141.

[56] Y. P. Menshikov, Zap. Vses. Miner. O-va, 1984, 113, 607-612.

[57] G. Artioli, A. Kvick, Eur. J. Mineral. 1990, 2, 749-760.

[58] C. Baerlocher, L. B. McCusker, D. H. Olson, Atlas of Zeolite Framework Types, Published On Behalf Of The Structure Commission Of The International Zeolite Association By Elsevier, 2007.

[59] R. M. Barrer, H. Villiger, Zeitschrift für Krist. 1969, 128, 352-370.

[60] C. Baerlocher, L. B. McCusker, "Database of Zeolite Structures http://www.iza-structure.org/databases/," 2018.

[61] S. Huber, G. Calzaferri, ChemPhysChem 2004, 5, 239-242.

[62] J. Yu, Y. Cui, C. Wu, Y. Yang, Z. Wang, M. O'Keeffe, B. Chen, G. Qian, Angew. Chem. Int. Ed. 2012, 51, 10542-10545; Angew. Chem. 2012, 124, 10694-10697.

[63] S. Inagaki, O. Ohtani, Y. Goto, K. Okamoto, M. Ikai, K. I. Yamanaka, T. Tani, T. Okada, Angew. Chem. Int. Ed. 2009, 48, 4042-4046; Angew. Chem. 2009, 121, 4102-4106.

[64] L. Sun, H. Xing, Z. Liang, J. Yu, R. Xu, Chem. Commun. 2013, 49, 11155-11157.

[65] A. Doménech, M. T. Doménech-Carbõ, C. Vidal-Lorenzo, M. L. V. De Agredos-Pascual, Angew. Chem. Int. Ed. 2012, 51, 700-703;

Angew. Chem. 2012, 124, 724-727.

[66] H. Berke, Chem. Soc. Rev. 2007, 36, 15-30.

[67] A. Tilocca, E. Fois, J. Phys. Chem. C 2009, 113, 8683-8687.

[68] R. Giustetto, K. Seenivasan, F. Bonino, G. Ricchiardi, S. Bordiga, M. R. Chierotti, R. Gobetto, J. Phys. Chem. C 2011, 115, 16764-16776.

[69] M. M. Lezhnina, T. Grewe, H. Stoehr, U. Kynast, Angew. Chem. Int. Ed. 2012, 51, 10652-10655; Angew. Chem. 2012, 124, 1080510809.

[70] E. Fois, A. Gamba, A. Tilocca, Microporous Mesoporous Mater. 2003, 57, 263-272.

[71] S. A. Hussain, R. A. Schoonheydt, Langmuir 2010, 26, 1187011877.

[72] D. A. Kunz, M. J. Leitl, L. Schade, J. Schmid, B. Bojer, U. T. Schwarz, G. A. Ozin, H. Yersin, J. Breu, Small 2015, 11, 792-796.

[73] M. Stöter, B. Biersack, S. Rosenfeldt, M. J. Leitl, H. Kalo, R. Schobert, H. Yersin, G. A. Ozin, S. Förster, J. Breu, Angew. Chem. Int. Ed. 2015, 54, 4963-4967; Angew. Chem. 2015, 127, 50475051.

[74] A. L. Costa, A. C. Gomes, R. C. Pereira, M. Pillinger, I. S. Gonçalves, M. Pineiro, J. S. Seixas de Melo, Langmuir 2018, 34, 453-464.

[75] E. D. Sloan, C. Koh, Clathrate Hydrates of Natural Gases, CRC Press, 2008.

[76] C. A. Koh, Chem. Soc. Rev. 2002, 31, 157-167.

[77] K. C. Hester, P. G. Brewer, Ann. Rev. Mar. Sci. 2009, 1, 303-327.

[78] E. D. Sloan, Nature 2003, 426, 353-359.

[79] L. Mandelcorn, Chem. Rev. 1959, 59, 827-839.

[80] R. M. Barrer, J. Chem. Soc. 1948, 0, 2158.

[81] R. M. Barrer, J. L. Whiteman, J. Chem. Soc. A 1967, 19-25.

[82] T. Bein, Chem. Mater. 1996, 8, 1636-1653.

[83] U. Simon, M. E. Franke, Microporous Mesoporous Mater. 2000, 41, $1-36$.
[84] A. Corma, Chem. Rev. 1995, 95, 559-614.

[85] C. S. Cundy, P. A. Cox, Chem. Rev. 2003, 103, 663-701.

[86] M. E. Davis, C. Saldarriaga, C. Montes, J. Garces, C. Crowdert, Nature 1988, 331, 698-699.

[87] L. B. McCusker, C. Baerlocher, E. Jahn, M. Bülow, Zeolites 1991, 11, 308-313.

[88] M. W. Anderson, O. Terasaki, T. Ohsuna, A. Philippou, S. P. MacKay, A. Ferreira, J. Rocha, S. Lidin, Nature 1994, 367, 347-351.

[89] C. L. Bowes, G. A. Ozin, Adv. Mater. 1996, 8, 13-28.

[90] N. Zheng, X. Bu, B. Wang, P. Feng, Science 2002, 298, 2366-2369.

[91] X. Bu, N. Zheng, P. Feng, Chem. Eur. J. 2004, 10, 3356-3362.

[92] P. Peng, B. U. Xianhui, N. Zheng, Acc. Chem. Res. 2005, 38, 293303.

[93] M. Zeuner, S. Pagano, W. Schnick, Angew. Chem. Int. Ed. 2011, 50, 7754-7775; Angew. Chem. 2011, 123, 7898-7920.

[94] F. Marlow, J. Caro, L. Werner, J. Kornatowski, J. Phys. Chem. 1993, 97, 11286-11290.

[95] U. Vietze, O. Krauß, F. Laeri, G. Ihlein, F. Schüth, B. Limburg, M. Abraham, Phys. Rev. Lett. 1998, 81, 4628-4631.

[96] T. Yanagisawa, T. Shimizu, K. Kuroda, C. Kato, Bull. Chem. Soc. Jpn. 1990, 63, 988-992.

[97] C. T. Kresge, M. E. Leonowicz, W. J. Roth, J. C. Vartuli, J. S. Beck, Nature 1992, 359, 710-712.

[98] C. Yoshina-Ishii, T. Asefa, N. Coombs, M. J. MacLachlan, G. A. Ozin, Chem. Commun. 1999, 0, 2539-2540.

[99] A. K. Cheetham, G. Férey, T. Loiseau, Angew. Chem. Int. Ed. 1999, 38, 3268-3292; Angew. Chem. 1999, 111, 3466-349.

[100] S. Kitagawa, R. Kitaura, S. Noro, Angew. Chem. Int. Ed. 2004, 43, 2334-2375; Angew. Chem. 2004, 116, 2388-2430.

[101] M. O'Keeffe, O. M. Yaghi, Chem. Rev. 2012, 112, 675-702.

[102] C. H. Hendon, A. J. Rieth, M. D. Korzyński, M. Dincă, ACS Cent. Sci. 2017, 3, 554-563.

[103] R. E. Morris, L. Brammer, Chem. Soc. Rev. 2017, 46, 5444-5462.

[104] B. Manna, A. K. Chaudhari, B. Joarder, A. Karmakar, S. K. Ghosh, Angew. Chem. Int. Ed. 2013, 52, 998-1002; Angew. Chem. 2013, 125, 1032-1036.

[105] W. H. Baur, Am. Mineral. 1964, 49, 697-704.

[106] G. Vezzalini, S. Quartieri, E. Galli, A. Alberti, G. Cruciani, A. Kvick, Zeolites 1997, 19, 323-325.

[107] C. Lengauer, G. Giester, E. Tillmanns, Mineral. Mag. 1997, 61, 591606.

[108] S. V. Krivovichev, Angew. Chem. Int. Ed. 2014, 53, 654-661; Angew. Chem. 2014, 126, 666-674.

[109] R. C. Rouse, D. R. Peacor, Am. Mineral. 1986, 71, 1494-1501.

[110] T. Iwamoto, J. Incl. Phenom. Mol. Recognit. Chem. 1996, 24, 61132.

[111] T. Iwamoto, S. Nishikiori, T. Kitazawa, H. Yuge, J. Chem. Soc. Dalt. Trans. 1997, 0, 4127-4136.

[112] T. Iwamoto, T. Kitazawa, S. Nishikiori, R. Kuroda, in Chem. Phys. Intercalation II (Proceedings Second NATO Adv. Study Inst. Chem. Phys. Intercalation, Chateau Bonas, June 29-July 9, 1992, 1993, pp. 325-332.

[113] T. Iwamoto, S. I. Nishikiori, T. Kitazawa, Supramol. Chem. 1995, 6, 179-186.

[114] N. A. Anurova, V. A. Blatov, G. D. Ilyushin, D. M. Proserpio, J. Phys. Chem. C 2010, 114, 10160-10170.

[115] C. Paris, M. Moliner, in Structure and Bonding, Springer, Berlin, Heidelberg, 2018, doi: https://doi.org/10.1007/430_2017_11.

[116] E. M. Flanigen, Pure Appl. Chem. 1980, 52, 2191-2211.

[117] J. A. Ripmeester, J. S. Tse, C. I. Ratcliffe, B. M. Powell, Nature 
1987, 325, 135-136.

[118] H. Lu, Y. T. Seo, J. W. Lee, I. Moudrakovski, J. A. Ripmeester, N. R. Chapman, R. B. Coffin, G. Gardner, J. Pohlman, Nature 2007, 445, 303-306.

[119] K. Momma, J. Phys. Condens. Matter 2014, 26, 103203.

[120] C. A. Geiger, E. Dachs, M. Nagashima, Am. Mineral. 2008, 93, 1179-1182.

[121] C. Dryzun, Y. Mastai, A. Shvalb, D. Avnir, J. Mater. Chem. 2009, 19, 2062-2069.

[122] J. Shin, H. Xu, S. Seo, P. Guo, J. G. Min, J. Cho, P. A. Wright, X. Zou, S. B. Hong, Angew. Chem. Int. Ed. 2016, 55, 4928-4932; Angew. Chem. 2016, 128, 5012-5016.

[123] P. Guo, J. Shin, A. G. Greenaway, J. G. Min, J. Su, H. J. Choi, L. Liu, P. A. Cox, S. B. Hong, P. A. Wright, X. Zou, Nature 2015, 524, $74-78$.

[124] B. Kamb, Science 1965, 148, 232-234

[125] M. Tribaudino, A. Artoni, C. Mavris, D. Bersani, P. P. Lottici, D. Belletti, Am. Mineral. 2008, 93, 88-94.

[126] G. Gottardi, E. Galli, Natural Zeolites, Springer Berlin, 2012

[127] T. Armbruster, M. E. Gunter, Rev. Mineral. Geochemistry 2001, 45, $1-67$.

[128] G. Vezzalini, R. Arletti, S. Quartieri, Acta Crystallogr. Sect. B 2014 70, 444-451.

[129] W. Depmeier, Part. Part. Syst. Charact. 2009, 26, 138-150.

[130] P. Macchi, Crystallogr. Rev. 2013, 19, 58-101.

[131] A. Cervellino, R. Frison, N. Masciocchi, A. Guagliardi, in X-Ray Neutron Tech. Nanomater. Charact., Springer Berlin Heidelberg, Berlin, Heidelberg, 2016, pp. 545-608.

[132] R. Gaillac, P. Pullumbi, K. A. Beyer, K. W. Chapman, D. A. Keen, T. D. Bennett, F.-X. Coudert, Nat. Mater. 2017, 16, 1149-1154.

[133] L. Gigli, R. Arletti, G. Tabacchi, M. Fabbiani, J. G. Vitillo, G. Martra, A. Devaux, I. Miletto, S. Quartieri, G. Calzaferri, E. Fois, J. Phys. Chem. C 2018, 122, 3401-3418.

[134] G. Kalantzopoulos, F. Lundvall, S. Checchia, A. Lind, D. Wragg, H Fjellvåg, B. Arstad, ChemPhysChem 2018, 19, 519-528.

[135] S. E. Ashbrook, D. M. Dawson, V. R. Seymour, Phys. Chem. Chem Phys. 2014, 16, 8223-8242.

[136] C. A. Fyfe, J. M. Thomas, J. Klinowski, G. C. Gobbi, Angew. Chem. Int. Ed. Engl. 1983, 22, 259-275; Angew. Chem. 1983, 95, 257-273.

[137] C. A. Fyfe, G. C. Gobbi, G. J. Kennedy, J. Phys. Chem. 1984, 88, 3248-3253.

[138] T. Yokoi, H. Mochizuki, S. Namba, J. N. Kondo, T. Tatsumi, J. Phys. Chem. C 2015, 119, 15303-15315.

[139] I. I. Ivanova, Y. G. Kolyagin, I. A. Kasyanov, A. V. Yakimov, T. O. Bok, D. N. Zarubin, Angew. Chem. Int. Ed. 2017, 56, 1534415347; Angew. Chem. 2017, 129, 15546-15549.

[140] A. Zheng, S.-B. Liu, F. Deng, Chem. Rev. 2017, 117, 12475-12531.

[141] C. Martineau, Solid State Nucl. Magn. Reson. 2014, 63, 1-12.

[142] A. Marchetti, J. Chen, Z. Pang, S. Li, D. Ling, F. Deng, X. Kong, Adv. Mater. 2017, 29, 1605895

[143] B. E. G. Lucier, Y. Zhang, Y. Huang, Concepts Magn. Reson. Part A 2017, e21410.

[144] A. J. Rossini, A. Zagdoun, M. Lelli, J. Canivet, S. Aguado, O. Ouari, P. Tordo, M. Rosay, W. E. Maas, C. Copéret, D. Farrusseng, L. Emsley, A. Lesage, Angew. Chem. Int. Ed. 2012, 51, 123127; Angew. Chem. 2012, 124, 127-131.

[145] E. Weiland, M.-A. Springuel-Huet, A. Nossov, A. Gédéon, Microporous Mesoporous Mater. 2016, 225, 41-65.

[146] J. A. Ripmeester, C. I. Ratcliffe, J. Phys. Chem. 1990, 94, 87738776.
[147] J. A. Ripmeester, C. I. Ratcliffe, J. Phys. Chem. 1990, 94, 76527656.

[148] S. Pawsey, I. Moudrakovski, J. Ripmeester, L.-Q. Wang, G. J. Exarhos, J. L. C. Rowsell, O. M. Yaghi, J. Phys. Chem. C 2007, 111, 6060-6067.

[149] P. Sozzani, A. Comotti, R. Simonutti, T. Meersmann, J. W. Logan, A Pines, Angew. Chem. Int. Ed. 2000, 39, 2695-2699; Angew. Chem. 2000, 112, 2807-2810.

[150] a Nossov, E. Haddad, F. Guenneau, A. Galarneau, F. Di Renzo, F. Fajula, A. Gedeon, J. Phys. Chem. B 2003, 107, 12456-12460.

[151] V. V. Terskikh, I. L. Moudrakovski, S. R. Breeze, S. Lang, C. I. Ratcliffe, J. A. Ripmeester, A. Sayari, Langmuir 2002, 18, 56535656.

[152] F. Guenneau, K. Panesar, A. Nossov, M.-A. Springuel-Huet, T. Azaïs, F. Babonneau, C. Tourné-Péteilh, J.-M. Devoisselle, A Gédéon, Phys. Chem. Chem. Phys. 2013, 15, 18805.

[153] D. I. Kolokolov, S. S. Arzumanov, D. Freude, J. Haase, A. G. Stepanov, J. Phys. Chem. C 2016, 120, 4993-5000.

[154] S. Bordiga, C. Lamberti, F. Bonino, A. Travert, F. Thibault-Starzyk, Chem. Soc. Rev. 2015, 44, 7262-7341.

[155] A. Zecchina, L. Marchese, S. Bordiga, C. Pazè, E. Gianotti, J. Phys. Chem. B 1997, 101, 10128-10135.

[156] A. Zecchina, C. Otero Areán, Chem. Soc. Rev. 1996, 25, 187-197.

[157] C. Otero Areán, A. A. Tsyganenko, E. Escalona Platero, E. Garrone, A. Zecchina, Angew. Chem. Int. Ed. 1998, 37, 3161-3163; Angew. Chem. 1998, 110, 3350-3353.

[158] S. Coluccia, L. Marchese, G. Martra, Microporous Mesoporous Mater. 1999, 30, 43-56.

[159] J. Canivet, V. Lysenko, J. Lehtinen, A. Legrand, F. M. Wisser, E. A Quadrelli, D. Farrusseng, ChemPhysChem 2017, 18, 2855-2858.

[160] S. H. Parekh, K. F. Domke, Chem. Eur. J. 2013, 19, 11822-11830.

[161] K. F. Domke, J. P. R. Day, G. Rago, T. A. Riemer, M. H. F. Kox, B. M. Weckhuysen, M. Bonn, Angew. Chem. Int. Ed. 2012, 51, 13431347; Angew. Chem. 2012, 124, 1371-1375.

[162] R. A. Schoonheydt, Chem. Soc. Rev. 2010, 39, 5051.

[163] A. Gasecka, L.-Q. Dieu, D. Brühwiler, S. Brasselet, J. Phys. Chem. B 2010, 114, 4192-4198.

[164] R. A. Schoonheydt, Angew. Chem. Int. Ed. 2008, 47, 9188-9191; Angew. Chem. 2008, 120, 9328-9331.

[165] B. M. Weckhuysen, Angew. Chem. Int. Ed. 2009, 48, 4910-4943; Angew. Chem. 2009, 121, 5008-5043.

[166] C. Blum, Y. Cesa, M. Escalante, V. Subramaniam, J. R. Soc. Interface 2009, 6, S35-S43.

[167] T. Bein, D. C. Lamb, J. Michaelis, ChemPhysChem 2012, 13, 8811095.

[168] S. C. C. Wiedemann, Z. Ristanovic, G. T. Whiting, V. R. Reddy Marthala, J. Kärger, J. Weitkamp, B. Wels, P. C. A. Bruijnincx, B. M. Weckhuysen, Chem. Eur. J. 2016, 22, 199-210.

[169] J. A. van Bokhoven, C. Lamberti, Coord. Chem. Rev. 2014, 277, 275-290.

[170] S. Smolders, K. A. Lomachenko, B. Bueken, A. Struyf, A. L. Bugaev, C. Atzori, N. Stock, C. Lamberti, M. B. J. Roeffaers, D. De Vos, ChemPhysChem 2018, 19, 373-378.

[171] V. Van Speybroeck, K. Hemelsoet, L. Joos, M. Waroquier, R. G. Bell, C. R. A. Catlow, Chem. Soc. Rev. 2015, 44, 7044-7111.

[172] C. R. A. Catlow, B. Smit, R. A. Van Santen, Computer Modelling of Microporous Materials, Elsevier, Amsterdam, NL, 2004.

[173] F. X. Coudert, A. H. Fuchs, Coord. Chem. Rev. 2015, 307, 211-236

[174] M. Fischer, J. R. B. Gomes, M. Jorge, Mol. Simul. 2014, 40, 537556. 
[175] G. Fraux, F.-X. Coudert, Chem. Commun. 2017, 53, 7211-7221.

[176] S. Jiang, Q. Song, A. Massey, S. Y. Chong, L. Chen, S. Sun, T. Hasell, R. Raval, E. Sivaniah, A. K. Cheetham, A. I. Cooper, Angew. Chem. Int. Ed. 2017, 56, 9391-9395; Angew. Chem. 2017, 129, 9519-9523.

[177] T. Ben, H. Ren, M. Shengqian, D. Cao, J. Lan, X. Jing, W. Wang, J. Xu, F. Deng, J. M. Simmons, S. Qiu, G. Zhu, Angew. Chem. Int. Ed. 2009, 48, 9457-9460; Angew. Chem. 2009, 121, 9621-9624.

[178] L. Vilà-Nadal, L. Cronin, Nat. Rev. Mater. 2017, 2, 17054.

[179] A. G. Slater, A. I. Cooper, Science 2015, 348, aaa8075.

[180] B. Smit, T. L. M. Maesen, Chem. Rev. 2008, 108, 4125-4184.

[181] P. Demontis, J. Karger, G. B. Suffritti, A. Tilocca, Phys. Chem. Chem. Phys. 2000, 2, 1455-1463.

[182] A. Torres-Knoop, D. Dubbeldam, ChemPhysChem 2015, 16, 20462067.

[183] M. Pfenniger, G. Calzaferri, ChemPhysChem 2000, 1, 211-217.

[184] J. Rybka, J. Kärger, U. Tallarek, ChemPhysChem 2017, 18, 2094 2102.

[185] J. Kärger, ChemPhysChem 2015, 16, 24-51.

[186] F. Li, D. P. Josephson, A. Stein, Angew. Chem. Int. Ed. 2011, 50, 360-388; Angew. Chem. 2011, 123, 378-409.

[187] N. Vogel, M. Retsch, C. A. Fustin, A. Del Campo, U. Jonas, Chem Rev. 2015, 115, 6265-6311.

[188] J. Čejka, A. Corma, S. Zones, Zeolites and Catalysis: Synthesis, Reactions and Applications, Volume 2, WILEY-VCH Verlag GmbH \& Co. KGaA, Weinheim, Germany, 2010.

[189] K. B. Yoon, Acc. Chem. Res. 2007, 40, 29-40.

[190] J. Cho, Y. Ishida, Adv. Mater. 2017, 29, 1605974.

[191] G. Tabacchi, E. Fois, G. Calzaferri, Angew. Chem. Int. Ed. 2015, 54, 11112-11116; Angew. Chem. 2015, 127, 11264-11268.

[192] W. Baur, R. X. Fischer, Microporous and Other Framework Materials with Zeolite-Type Structures, SPRINGER-VERLAG BERLIN AN, 2017.

[193] R. Szostak, Molecular Sieves - Springer, Springer Netherlands, 1989.

[194] D. S. Coombs, A. Alberti, T. Armbruster, G. Artioli, C. Colella, E. Galli, J. D. Grice, F. Liebau, E. H. Nickel, E. Passaglia, D. R. Peacor, S. Quartieri, R. Rinaldi, M. Ross, R. A. Sheppard, G. Vezzalini, Can. Mineral. 1997, 35, 1571-1606.

[195] M. M. J. Treacy, M. D. Foster, Microporous Mesoporous Mater. 2009, 118, 106-114.

[196] V. A. Blatov, O. Delgado-Friedrichs, M. O’Keeffe, D. M. Proserpio, Acta Crystallogr. Sect. A Found. Crystallogr. 2007, 63, 418-425.

[197] G. Sastre, A. Corma, J. Phys. Chem. C 2009, 113, 6398-6405.

[198] Y. Li, J. Yu, Chem. Rev. 2014, 114, 7268-7316.

[199] M. Moliner, C. Martínez, A. Corma, Angew. Chem. Int. Ed. 2015, 54, 3560-3579; Angew. Chem. 2015, 127, 3630-3649.

[200] W. J. Roth, P. Nachtigall, R. E. Morris, J. Čejka, Chem. Rev. 2014 114, 4807-4837.

[201] J. Choi, S. Ghosh, Z. Lai, M. Tsapatsis, Angew. Chem. Int. Ed. 2006, 45, 1154-1158; Angew. Chem. 2006, 118, 1172-1176.

[202] J. A. Boscoboinik, X. Yu, B. Yang, F. D. Fischer, R. Wodarczyk, M. Sierka, S. Shaikhutdinov, J. Sauer, H. J. Freund, Angew. Chem. Int. Ed. 2012, 51, 6005-6008; Angew. Chem. 2012, 124, 6107-6111.

[203] U. Díaz, A. Corma, Dalt. Trans. 2014, 43, 10292.

[204] S. Zanardi, A. Alberti, G. Cruciani, A. Corma, V. Fornés, M. Brunelli, Angew. Chem. Int. Ed. 2004, 43, 4933-4937; Angew. Chem. 2004, 116, 5041-5045.

[205] M. Hartmann, Angew. Chem. Int. Ed. 2004, 43, 5880-5882; Angew. Chem. 2004, 116, 6004-6006.
[206] Y. Wei, T. E. Parmentier, K. P. de Jong, J. Zečević, Chem. Soc. Rev. 2015, 44, 7234-7261.

[207] M. A. Snyder, M. Tsapatsis, Angew. Chem. Int. Ed. 2007, 46, 7560 7573; Angew. Chem. 2007, 119, 7704-7717.

[208] G. A. Ozin, http://www.artnanoinnovations.com/ozin/Nanochemistry Views.pdf 2012.

[209] F. C. Hendriks, J. E. Schmidt, J. A. Rombouts, K. Lammertsma, P. C. A. Bruijnincx, B. M. Weckhuysen, Chem. Eur. J. 2017, 23, 63056314

[210] J. E. Schmidt, F. C. Hendriks, M. Lutz, L. C. Post, D. Fu, dr. B. M. Weckhuysen, ChemPhysChem 2018, 19, 367-372.

[211] C. Weidenthaler, R. X. Fischer, R. D. Shannon, O. Medenbach, J. Phys. Chem. 1994, 98, 12687-12694.

[212] S. Sklenak, J. Dědeček, C. Li, B. Wichterlová, V. Gábová, M. Sierka J. Sauer, Angew. Chem. Int. Ed. 2007, 46, 7286-7289; Angew. Chem. 2007, 119, 7424-7427.

[213] A. R. Ruiz-Salvador, R. Grau-Crespo, A. E. Gray, D. W. Lewis, J. Solid State Chem. 2013, 198, 330-336.

[214] L. Grajciar, C. O. Areán, A. Pulido, P. Nachtigall, Phys. Chem. Chem. Phys. 2010, 12, 1497-506.

[215] R. Rohling, E. Hensen, E. A. Pidko, ChemPhysChem 2018, 19, 446-458.

[216] M. Fischer, Phys. Chem. Chem. Phys. 2016, 18, 15738-15750.

[217] M. Fischer, Phys. Chem. Chem. Phys. 2015, 17, 25260-25271.

[218] J. D. Gale, Solid State Sci. 2006, 8, 234-240.

[219] E. Spanó, G. Tabacchi, A. Gamba, E. Fois, J. Phys. Chem. B 2006, 110, 21651-21661.

[220] A. Gamba, G. Tabacchi, E. Fois, J. Phys. Chem. A 2009, 113, 15006-15015.

[221] M. Signorile, A. Damin, F. Bonino, V. Crocellà, G. Ricchiardi, C. Lamberti, S. Bordiga, J. Phys. Chem. C 2018, 122, 1612-1621.

[222] C. Betti, E. Fois, E. Mazzucato, C. Medici, S. Quartieri, G. Tabacchi, G. Vezzalini, V. Dmitriev, Microporous Mesoporous Mater. 2007, 103, 190-209.

[223] E. Fois, A. Gamba, C. Medici, G. Tabacchi, S. Quartieri, E. Mazzucato, R. Arletti, G. Vezzalini, V. Dmitriev, Microporous Mesoporous Mater. 2008, 115, 267-280.

[224] D. W. Breck, Zeolite Molecular Sieves: Structure, Chemistry, and Use, Wiley, 1973.

[225] A. Zecchina, F. Geobaldo, G. Spoto, S. Bordiga, G. Ricchiardi, R Buzzoni, G. Petrini, J. Phys. Chem. 1996, 100, 16584-16599.

[226] M. Sierka, U. Eichler, J. Datka, J. Sauer, J. Phys. Chem. B 1998, 102, 10468-10468.

[227] T. Demuth, J. Hafner, L. Benco, H. Toulhoat, J. Phys. Chem. B 2000, 104, 4593-4607.

[228] L. Benco, J. Catal. 2002, 205, 147-156.

[229] E. Fois, A. Gamba, G. Tabacchi, J. Phys. Chem. B 1998, 102 3974-3979.

[230] A. V. Larin, D. N. Trubnikov, D. P. Vercauteren, Int. J. Quantum Chem. 2005, 102, 971-979.

[231] R. Fricke, H. Kosslick, G. Lischke, M. Richter, Chem. Rev. 2000, 100, 2303-2405.

[232] R. M. Barrer, J. W. Baynham, F. W. Bultitude, W. M. Meier, J. Chem Soc. 1959, 36, 195-208.

[233] C. Otero Areán, G. Turnes Palomino, F. Geobaldo, A. Zecchina, J. Phys. Chem. 1996, 100, 6678-6690.

[234] E. Fois, A. Gamba, G. Tabacchi, Phys. Chem. Chem. Phys. 1999, 1, 531-536.

[235] Z. Gabelica, J. B. Nagy, P. Bodart, G. Debras, Chem. Lett. 1984, 13, 1059-1062. 
[236] G. Valerio, J. Ple'vert, A. Goursot, F. di Renzo, Phys. Chem. Chem. Phys. 2000, 2, 1091-1094.

[237] A. Goursot, D. Berthomieu, Magn. Reson. Chem. 2004, 42, S180S186.

[238] F. Trudu, G. Tabacchi, A. Gamba, E. Fois, J. Phys. Chem. A 2007, 111, 11626-37.

[239] C. Fild, D. F. Shantz, R. F. Lobo, H. Koller, Phys. Chem. Chem Phys. 2000, 2, 3091-3098.

[240] S. J. Hwang, C. Y. Chen, S. I. Zones, J. Phys. Chem. B 2004, 108 18535-18546.

[241] F. Trudu, G. Tabacchi, A. Gamba, E. Fois, J. Phys. Chem. C 2008 112, 15394-15401.

[242] E. Fois, A. Gamba, F. Trudu, G. Tabacchi, Nuovo Cim. della Soc. Ital. di Fis. B 2008, 123, 1567-1574.

[243] H. Sheng, E. P. Schreiner, W. Zheng, R. F. Lobo, ChemPhysChem 2018, 19, 504-511.

[244] A. J. Martín, S. Mitchell, O. Scholder, R. Verel, R. Hauert, L. Bernard, C. Jensen, M. Schwefer, J. Pérez-Ramírez, ChemPhysChem 2018, 19, 437-445.

[245] S. T. Wilson, B. M. Lok, C. A. Messina, T. R. Cannan, E. M. Flanigen, J. Am. Chem. Soc 1982, 104, 1146.

[246] H. O. Pastore, S. Coluccia, L. Marchese, Annu. Rev. Mater. Res. 2005, 35, 351-395.

[247] A. Corma, U. Diaz, M. E. Domine, V. Fornés, Chem. Commun. 2000, 137-138.

[248] E. A. Eilertsen, F. Giordanino, C. Lamberti, S. Bordiga, A. Damin, F. Bonino, U. Olsbye, K. P. Lillerud, Chem. Commun. 2011, 47, 11867-9.

[249] J. Jiang, J. Yu, A. Corma, Angew. Chem. Int. Ed. 2010, 49, 31203145; Angew. Chem. 2010, 122, 3186-3212.

[250] Z. Gabelica, J.-L. Guth, Angew. Chem. Int. Ed. Engl. 1989, 28, 8183; Angew. Chem. 1989, 101, 3186-3212.

[251] A. Corma, M. T. Navarro, F. Rey, J. Rius, S. Valencia, Angew. Chem. Int. Ed. 2001, 40, 2277-2280; Angew. Chem. 2001, 113, 2337-2340.

[252] P. Eliášová, M. Opanasenko, P. S. Wheatley, M. Shamzhy, M. Mazur, P. Nachtigall, W. J. Roth, R. E. Morris, J. Čejka, Chem. Soc. Rev. 2015, 44, 7177-7206.

[253] R. L. Bedard, S. T. Wilson, L. D. Vail, J. M. Bennett, E. M. Flanigen, Stud. Surf. Sci. Catal. 1989, 49, 375-387.

[254] A. Corma, M. E. Davis, ChemPhysChem 2004, 5, 304-313.

[255] G. Bellussi, E. Montanari, E. Di Paola, R. Millini, A. Carati, C. Rizzo, W. O'Neil Parker, M. Gemmi, E. Mugnaioli, U. Kolb, S. Zanardi, Angew. Chem. Int. Ed. 2012, 51, 666-669; Angew. Chem. 2012, 124, 690-693.

[256] G. Bellussi, R. Millini, E. Montanari, A. Carati, C. Rizzo, W. O. Parker, G. Cruciani, A. de Angelis, L. Bonoldi, S. Zanardi, Chem. Commun. 2012, 48, 7356

[257] E. Fois, A. Gamba, E. Spanò, Phys. Chem. Chem. Phys. 2001, 3, 1877-1882.

[258] L. Wang, R.-J. Xie, T. Suehiro, T. Takeda, N. Hirosaki, Chem. Rev. 2018, acs.chemrev.7b00284.

[259] A. Marchuk, W. Schnick, Angew. Chem. Int. Ed. 2015, 54, 23832387; Angew. Chem. 2015, 127, 2413-2417.

[260] P. Bielec, O. Janka, T. Block, R. Pöttgen, W. Schnick, Angew. Chem. Int. Ed. 2018, 57, 2409-2412; Angew. Chem. 2018, 130, 2433-2436.

[261] P. Bielec, W. Schnick, Angew. Chem. Int. Ed. 2017, 56, 4810-4813 Angew. Chem. 2017, 129, 4888-4891.

[262] N. C. Jeong, M. H. Lee, K. B. Yoon, Angew. Chem. Int. Ed. 2007, 46,
5868-5872; Angew. Chem. 2007, 119, 5972-5976.

[263] N. C. Jeong, H. Lim, H. Cheong, K. B. Yoon, Angew. Chem. Int. Ed. 2011, 50, 8697-8701; Angew. Chem. 2011, 123, 8856-8860.

[264] S. J. Datta, K. B. Yoon, Catal. Today 2013, 204, 60-65.

[265] S. Galioglu, M. Isler, Z. Demircioglu, M. Koc, F. Vocanson, N. Destouches, R. Turan, B. Akata, Microporous Mesoporous Mater. 2014, 196, 136-144.

[266] J. Lin, Y. Dong, Q. Zhang, D. Hu, N. Li, L. Wang, Y. Liu, T. Wu, Angew. Chem. Int. Ed. 2015, 54, 5103-5107; Angew. Chem. 2015, 127, 5192-5196.

[267] C. H. Hendon, K. T. Butler, A. M. Ganose, Y. Román-Leshkov, D. O. Scanlon, G. A. Ozin, A. Walsh, Chem. Mater. 2017, 29, 3663-3670.

[268] D. D. Hu, J. Lin, Q. Zhang, J. N. Lu, X. Y. Wang, Y. W. Wang, F. Bu L. F. Ding, L. Wang, T. Wu, Chem. Mater. 2015, 27, 4099-4104.

[269] D.-D. Hu, L. Wang, J. Lin, F. Bu, T. Wu, J. Mater. Chem. C 2015, 3 , 11747-11753.

[270] X. Chen, X. Bu, Q. Lin, C. Mao, Q.-G. Zhai, Y. Wang, P. Feng, Chem. Eur. J. 2017, 23, 11913-11919.

[271] M. Wiebcke, J. Chem. Soc. Chem. Commun. 1991, 1507

[272] K. E. Lazzeri, G. E. Bebout, C. A. Geiger, Am. Mineral. 2017, 102, 686-689.

[273] C. A. Fyfe, H. Gies, J. Incl. Phenom. Mol. Recognit. Chem. 1990, 8 235-239.

[274] M. Tribaudino, G. D. Gatta, Y. Lee, Microporous Mesoporous Mater. 2010, 129, 267-273.

[275] B. A. Kolesov, C. A. Geiger, Am. Mineral. 2003, 88, 1364-1368.

[276] K. E. Lazzeri, G. E. Bebout, C. A. Geiger, Am. Mineral. 2017, 102, 686-689.

[277] A. Y. Likhacheva, S. V. Goryainov, Y. V. Seryotkin, K. D. Litasov, K. Momma, Microporous Mesoporous Mater. 2016, 224, 100-106.

[278] K. Momma, T. Ikeda, K. Nishikubo, N. Takahashi, C. Honma, M. Takada, Y. Furukawa, T. Nagase, Y. Kudoh, Nat. Commun. 2011, 2, 196.

[279] V. V. Struzhkin, B. Militzer, W. L. Mao, H. K. Mao, R. J. Hemley, Chem. Rev. 2007, 107, 4133-4151.

[280] A. W. C. van den Berg, C. O. Areán, Chem. Commun. 2008, 73 668-681.

[281] K. A. Udachin, C. I. Ratcliffe, J. A. Ripmeester, J. Supramol. Chem. 2002, 2, 405-408.

[282] A. Falenty, T. C. Hansen, W. F. Kuhs, Nature 2014, 516, 231-233.

[283] G. McLaurin, K. Shin, S. Alavi, J. A. Ripmeester, Angew. Chem. Int Ed. 2014, 53, 10429-10433; Angew. Chem. 2014, 126, 1059710601.

[284] K. S. Smirnov, Phys. Chem. Chem. Phys. 2017, 19, 23095-23105.

[285] R. M. Barrer, G. C. Bratt, J. Phys. Chem. Solids 1960, 12, 130-145.

[286] K. A. Udachin, C. I. Ratcliffe, J. A. Ripmeester, Angew. Chem. Int Ed. 2001, 40, 1303-1305; Angew. Chem. 2001, 113, 1343-1345.

[287] A. V. Kurnosov, V. Y. Komarov, V. I. Voronin, A. E. Teplykh, A. Y. Manakov, Angew. Chem. Int. Ed. 2004, 43, 2922-2924; Angew. Chem. 2004, 116, 2982-2984.

[288] J. S. Loveday, R. J. Nelmes, Phys. Chem. Chem. Phys. 2008, 10, 937-950.

[289] A. Y. Manakov, A. Y. Likhacheva, V. A. Potemkin, A. G. Ogienko, A. V. Kurnosov, A. I. Ancharov, ChemPhysChem 2011, 12, 2476-2484.

[290] Y. A. Dyadin, É. G. Larionov, E. Y. Aladko, A. Y. Manakov, F. V. Zhurko, T. V. Mikina, V. Y. Komarov, E. V. Grachev, J. Struct. Chem. 1999, 40, 790-795.

[291] D. W. Davidson, Y. P. Handa, C. I. Ratcliffe, J. S. Tse, B. M. Powell, Nature 1984, 311, 142-143.

[292] K. A. Udachin, C. I. Ratcliffe, J. A. Ripmeester, J. Phys. Chem. B 
2001, 105, 4200-4204.

[293] J. H. van der Waals, J. C. Platteeuw, Adv. Chem. Phys. 1959, 2, 1

[294] O. Hassel, J. Hvoslef, Acta Chem. Scand. 1954, 5, 873.

[295] A. C. Legon, Angew. Chem. Int. Ed. 1999, 38, 2686-2714; Angew. Chem. 1999, 111, 2850-2880.

[296] P. Metrangolo, G. Resnati, Chem. Eur. J. 2001, 7, 2511-2519.

[297] P. Politzer, J. S. Murray, ChemPhysChem 2013, 14, 278-294.

[298] G. R. Desiraju, P. S. Ho, L. Kloo, A. C. Legon, R. Marquardt, P. Metrangolo, P. Politzer, G. Resnati, K. Rissanen, Pure Appl. Chem. 2013, 85, 1711-1713.

[299] K. A. Udachin, S. Alavi, J. A. Ripmeester, J. Phys. Chem. C 2013, $117,14176-14182$.

[300] K. W. Allen, G. A. Jeffrey, J. Chem. Phys. 1963, 38, 2304-2305.

[301] D. Ochoa-Resendiz, F. A. Batista-Romero, R. HernándezLamoneda, J. Chem. Phys. 2016, 145, 161104.

[302] R. Franklin-Mergarejo, J. Rubayo-Soneira, N. Halberstadt, K. C. Janda, V. A. Apkarian, J. Chem. Phys. 2016, 144, 54307.

[303] H. Dureckova, T. K. Woo, K. A. Udachin, J. A. Ripmeester, S. Alavi, Faraday Discuss. 2017, 203, 61-77.

[304] A. M. Guloy, R. Ramlau, Z. Tang, W. Schnelle, M. Baitinger, Y. Grin, Nature 2006, 443, 320-323.

[305] L. Senadheera, M. S. Conradi, J. Phys. Chem. B 2007, 111, 1209712102.

[306] S. Alavi, J. A. Ripmeester, Angew. Chem. Int. Ed. 2007, 46, 61026105; Angew. Chem. 2007, 119, 6214-6217.

[307] A. N. Salamatin, A. Falenty, T. C. Hansen, W. F. Kuhs, Energy \& Fuels 2015, 29, 5681-5691.

[308] A. Falenty, A. N. Salamatin, W. F. Kuhs, J. Phys. Chem. C 2013 117, 8443-8457.

[309] U. Ranieri, M. M. Koza, W. F. Kuhs, S. Klotz, A. Falenty, P. Gillet, L. E. Bove, Nat. Commun. 2017, 8, 1076.

[310] J. Zhu, S. Du, X. Yu, J. Zhang, H. Xu, S. C. Vogel, T. C. Germann, J. S. Francisco, F. Izumi, K. Momma, Y. Kawamura, C. Jin, Y. Zhao, Nat. Commun. 2014, 5, ncomms5128.

[311] S. Takeya, H. Fujihisa, H. Yamawaki, Y. Gotoh, R. Ohmura, S. Alavi, J. A. Ripmeester, Angew. Chem. Int. Ed. 2016, 55, 9287-9291; Angew. Chem. 2016, 128, 9433-9437.

[312] M. Hiratsuka, R. Ohmura, A. K. Sum, S. Alavi, K. Yasuoka, Phys. Chem. Chem. Phys. 2015, 17, 12639-12647.

[313] K. Shin, I. L. Moudrakovski, M. D. Davari, S. Alavi, C. I. Ratcliffe, J. A. Ripmeester, CrystEngComm 2014, 16, 7209-7217.

[314] K. Shin, I. L. Moudrakovski, C. I. Ratcliffe, J. A. Ripmeester, Angew. Chem. Int. Ed. 2017, 56, 6171-6175; Angew. Chem. 2017, 129, 6267-6271.

[315] J. A. Ripmeester, S. Alavi, Curr. Opin. Solid State Mater. Sci. 2016, 20, 344-351.

[316] Z. M. Aman, C. A. Koh, Chem. Soc. Rev. 2016, 45, 1678-1690.

[317] D. W. Davidson, L. D. Calvert, F. Lee, J. A. Ripmeester, Inorg. Chem. 1981, 20, 2013-2016.

[318] M. Wiebcke, D. Mootz, Zeitschrift für Krist. 1986, 177, 291-300.

[319] R. K. McMullan, T. C. W. Mak, G. A. Jeffrey, J. Chem. Phys. 1966, 44, 2338-2345

[320] E. V. Sokolova, V. B. Rybakov, L. A. Pautov, Sov. Phys. Dokl. Vol 36, p.2671991, 36, 267

[321] G. Buscarino, A. Alessi, S. Agnello, B. Boizot, F. M. Gelardi, R. Boscaino, Phys. Chem. Chem. Phys. 2014, 16, 13360.

[322] F. Messina, M. Todaro, G. Buscarino, L. Vaccaro, M. Cannas, F. M. Gelardi, Phys. Chem. Miner. 2016, 43, 171-179.

[323] Y. A. Dmitriev, G. Buscarino, N. P. Benetis, J. Phys. Chem. A 2016, 120, 6155-6169.
[324] G. Flachenecker, V. A. Ermoshin, V. Engel, R. Neder, G. Wirnsberger, A. Materny, Phys. Chem. Chem. Phys. 2003, 5, 865876

[325] H. van Koningsveld, H. Gies, Zeitschrift für Krist. - Cryst. Mater 2004, 219, 637-643.

[326] H. Gies, F. Liebau, H. Gerke, Angew. Chem. Int. Ed. Engl. 1982, 21, 206-207; Angew. Chem. 1982, 94, 214-215

[327] A. V. Shevelkov, K. Kovnir, Struct. Bond. 2011, 139, 97-142.

[328] N. A. Wagner, R. Raghavan, R. Zhao, Q. Wei, X. Peng, C. K. Chan, ChemElectroChem 2014, 1, 347-353.

[329] J. Pérez-Ramírez, S. Abelló, L. A. Villaescusa, A. Bonilla, Angew Chem. Int. Ed. 2008, 47, 7913-7917; Angew. Chem. 2008, 120, 8031-8035.

[330] J. Gryko, P. F. McMillan, R. F. Marzke, G. K. Ramachandran, D. Patton, S. K. Deb, O. F. Sankey, Phys. Rev. B - Condens. Matter Mater. Phys. 2000, 62, R7707-R7710.

[331] T. F. Fässler, Angew. Chem. Int. Ed. 2007, 46, 2572-2575; Angew. Chem. 2007, 120, 2624-2628.

[332] G. S. Nolas, M. Beekman, J. Gryko, G. A. Lamberton, T. M. Tritt, P. F. McMillan, Appl. Phys. Lett. 2003, 82, 910-912.

[333] H. Zhang, W. Peng, G. Mu, T. Hu, F. Huang, X. Xie, Chem. Eur. J. 2017, 23, 9505-9516.

[334] Q. Fang, G. Zhu, M. Xue, J. Sun, Y. Wei, S. Qui, R. Xu, Angew. Chem. Int. Ed. 2005, 44, 3845-3848; Angew. Chem. 2005, 117 3913-3916.

[335] M. A. Kirsanova, A. V. Olenev, A. M. Abakumov, M. A. Bykov, A. V. Shevelkov, Angew. Chem. Int. Ed. 2011, 50, 2371-2374; Angew. Chem. 2011, 123, 2419-2422.

[336] A. J. Karttunen, T. F. Fässler, ChemPhysChem 2013, 14, 18071817.

[337] U. Schwarz, A. Wosylus, B. Böhme, M. Baitinger, M. Hanfland, Y. Grin, Angew. Chem. Int. Ed. 2008, 47, 6790-6793; Angew. Chem. 2008, 120, 6895-6898.

[338] M. Beekman, G. S. Nolas, J. Mater. Chem. 2008, 18, 842-851.

[339] C. Gatti, L. Bertini, N. P. Blake, B. B. Iversen, Chem. Eur. J. 2003, 9 , 4556-4568.

[340] R. Nesper, K. Vogel, P. E. Blöchl, Angew. Chem. Int. Ed. Engl. 1993 32, 701-703; Angew. Chem. 1993, 105, 786-788.

[341] I. A. Baburin, D. M. Proserpio, V. A. Saleev, A. V. Shipilova, Phys Chem. Chem. Phys. 2015, 17, 1332-1338.

[342] R. Car, M. Parrinello, Phys. Rev. Lett. 1985, 55, 2471-2474.

[343] L. Zhu, H. Liu, R. E. Cohen, R. Hoffmann, T. A. Strobel, http://arxiv.org/abs/1708.03483 2017.

[344] J. L. Schlenker, F. G. Dwyer, E. E. Jenkins, W. J. Rohrbaugh, G. T. Kokotailo, W. M. Meier, Nature 1981, 294, 340-342.

[345] G. Férey, C. Serre, C. Mellot-Draznieks, F. Millange, S. Surblé, J. Dutour, I. Margiolaki, Angew. Chem. Int. Ed. 2004, 43, 6296-6301; Angew. Chem. 2004, 116, 6456-6461.

[346] D. Kim, H. Lee, Korean J. Chem. Eng. 2016, 33, 1977-1988.

[347] Y. Wan, D. Zhao, Chem. Rev. 2007, 107, 2821-2860.

[348] U. Ciesla, F. Schüth, Microporous Mesoporous Mater. 1999, 27, 131-149.

[349] K. Ariga, A. Vinu, Y. Yamauchi, Q. Ji, J. P. Hill, Bull. Chem. Soc. Jpn. 2012, 85, 1-32.

[350] F. Hoffmann, M. Cornelius, J. Morell, M. Fröba, Angew. Chem. Int Ed. 2006, 45, 3216-3251; Angew. Chem. 2006, 118, 3290-3328.

[351] A. Thomas, Angew. Chem. Int. Ed. 2010, 49, 8328-8344; Angew. Chem. 2010, 122, 8506-8523.

[352] W. J. Hunks, G. A. Ozin, J. Mater. Chem. 2005, 15, 3716.

[353] W. Wang, J. E. Lofgreen, G. A. Ozin, Small 2010, 6, 2634-2642. 
[354] H. H. P. Yiu, P. A. Wright, J. Mater. Chem. 2005, 15, 3690.

[355] F. Pitzalis, M. Monduzzi, A. Salis, Microporous Mesoporous Mater. 2017, 241, 145-154.

[356] D. I. Fried, F. J. Brieler, M. Fröba, ChemCatChem 2013, 5, 862-884.

[357] A. L. Doadrio, J. M. Sánchez-Montero, J. C. Doadrio, A. J. Salinas, M. Vallet-Regí, Eur. J. Pharm. Sci. 2017, 97, 1-8.

[358] A. Takai, Y. Doi, Y. Yamauchi, K. Kuroda, J. Phys. Chem. C 2010 114, 7586-7593.

[359] P. Innocenzi, L. Malfatti, T. Kidchob, P. Falcaro, Chem. Mater. 2009 $21,2555-2564$

[360] F. Hoffmann, M. Fröba, Chem. Soc. Rev. 2011, 40, 608-620.

[361] G. J. A. A. Soler-Illia, P. Innocenzi, Chem. Eur. J. 2006, 12, 44784494.

[362] G. Paul, G. E. Musso, E. Bottinelli, M. Cossi, L. Marchese, G. Berlier, ChemPhysChem 2017, 18, 839-849.

[363] G. E. Musso, E. Bottinelli, L. Celi, G. Magnacca, G. Berlier, Phys. Chem. Chem. Phys. 2015, 17, 13882-13894.

[364] N. Gartmann, D. Brühwiler, Angew. Chem. Int. Ed. 2009, 48, 63546356; Angew. Chem. 2009, 121, 6472-6475.

[365] D. Brühwiler, G. Calzaferri, T. Torres, J. H. Ramm, N. Gartmann, L.Q. Dieu, I. López-Duarte, M. V. Martínez-Díaz, J. Mater. Chem. 2009, 19, 8040-8067.

[366] N. Zucchetto, D. Brühwiler, Dalt. Trans. 2016, 45, 14363-14369

[367] Z. Li, J. C. Barnes, A. Bosoy, J. F. Stoddart, J. I. Zink, Chem. Soc. Rev. 2012, 41, 2590.

[368] C. Park, K. Oh, S. C. Lee, C. Kim, Angew. Chem. Int. Ed. 2007, 46, 1455-1457; Angew. Chem. 2007, 119, 1477-1479.

[369] C. Argyo, V. Weiss, C. Bräuchle, T. Bein, Chem. Mater. 2014, 26 , 435-451.

[370] T. D. Nguyen, H.-R. Tseng, P. C. Celestre, A. H. Flood, Y. Liu, J. F. Stoddart, J. I. Zink, Proc. Natl. Acad. Sci. U. S. A. 2005, 102, 10029-34.

[371] C. Wang, Z. Li, D. Cao, Y. L. Zhao, J. W. Gaines, O. A. Bozdemir, M. W. Ambrogio, M. Frasconi, Y. Y. Botros, J. I. Zink, J. F. Stoddart, Angew. Chem. Int. Ed. 2012, 51, 5460-5465; Angew. Chem. 2012, 124, 5556-5561.

[372] T. Asefa, M. J. MacLachan, N. Coombs, G. A. Ozin, Nature 1999, 402, 867-871.

[373] S. Inagaki, S. Guan, Y. Fukushima, T. Ohsuna, O. Terasaki, J. Am. Chem. Soc. 1999, 121, 9611-9614.

[374] Brian J. Melde, Brian T. Holland, A. Christopher F. Blanford, A Stein, Chem. Mater 1999, 11, 3302-3308.

[375] T. Asefa, M. J. MacLachlan, H. Grondey, N. Coombs, G. A. Ozin, Angew. Chem. Int. Ed. 2000, 39, 1808-1811; Angew. Chem. 2000, $112,1878-1881$.

[376] L. Grösch, Y. J. Lee, F. Hoffmann, M. Fröba, Chem. Eur. J. 2015, 21, 331-346.

[377] T. Martin, A. Galarneau, F. Di Renzo, F. Fajula, D. Plee, Angew. Chem. Int. Ed. 2002, 41, 2590-2592; Angew. Chem. 2002, 114, 2702-2704.

[378] A. Corma, U. Díaz, Chem. Eur. J. 2018, DOI: 10.1002/chem.201704185.

[379] A. Rimola, D. Costa, M. Sodupe, J.-F. Lambert, P. Ugliengo, Chem. Rev. 2013, 113, 4216-4313.

[380] B. Coasne, A. Galarneau, R. J. M. Pellenq, F. Di Renzo, Chem. Soc. Rev. 2013, 42, 4141-71.

[381] E. Fois, A. Gamba, G. Tabacchi, S. Coluccia, G. Martra, J. Phys. Chem. B 2003, 107, 10767-10772.

[382] E. Fois, A. Gamba, G. Tabacchi, S. Coluccia, G. Martra, J. Porous Mater. 2007, 14, 339-347.
[383] G. Tabacchi, E. Gianotti, E. Fois, G. Martra, L. Marchese, S. Coluccia, A. Gamba, J. Phys. Chem. C 2007, 111, 4946-4955.

[384] E. Fois, G. Tabacchi, D. Barreca, A. Gasparotto, E. Tondello, Angew. Chem. Int. Ed. 2010, 49, 1944-1948; Angew. Chem. 2010, 122, 1988-1992.

[385] G. Tabacchi, E. Fois, D. Barreca, A. Gasparotto, Phys. Status Solidi A 2014, 211, 251-259.

[386] A. Rimola, P. Ugliengo, M. Sodupe, Comput. Theor. Chem. 2015 1074, 168-177.

[387] S. Khanniche, D. Mathieu, F. Pereira, L. Hairault, Microporous Mesoporous Mater. 2017, 250, 158-169.

[388] M. Corno, M. Delle Piane, P. Choquet, P. Ugliengo, Phys. Chem. Chem. Phys. 2017, 19, 7793-7806.

[389] A. Gignone, M. Delle Piane, M. Corno, P. Ugliengo, B. Onida, J. Phys. Chem. C 2015, 119, 13068-13079.

[390] A. Rimola, M. Sodupe, P. Ugliengo, J. Phys. Chem. C 2009, 113, 5741-5750.

[391] M. Trachta, O. Bludský, M. Rubeš, Comput. Theor. Chem. 2017, 1117, 100-107.

[392] L. Giussani, G. Tabacchi, E. Gianotti, S. Coluccia, E. Fois, Philos. Trans. A. Math. Phys. Eng. Sci. 2012, 370, 1463-77.

[393] X. Y. Guo, T. Watermann, D. Sebastiani, J. Phys. Chem. B 2014, 118, 10207-10213.

[394] A. Kommu, J. K. Singh, J. Phys. Chem. C 2017, 121, 7867-7880.

[395] L. Giussani, E. Fois, E. Gianotti, G. Tabacchi, A. Gamba, S. Coluccia, ChemPhysChem 2010, 11, 1757-1762.

[396] L. Giussani, E. Fois, E. Gianotti, G. Tabacchi, A. Gamba, S. Coluccia, Nuovo Cim. della Soc. Ital. di Fis. B 2008, 123, 14771483.

[397] H. G. Manyar, E. Gianotti, Y. Sakamoto, O. Terasaki, S. Coluccia, S Tumbiolo, J. Phys. Chem. C 2008, 112, 18110-18116.

[398] A. R. Abdel Hamid, R. Mhanna, P. Catrou, Y. Bulteau, R. Lefort, D. Morineau, J. Phys. Chem. C 2016, 120, 11049-11053.

[399] R. Mhanna, A. R. Abdel Hamid, S. Dutta, R. Lefort, L. Noirez, B. Frick, D. Morineau, J. Chem. Phys. 2017, 146, 24501.

[400] A. R. Abdel Hamid, R. Mhanna, R. Lefort, A. Ghoufi, C. AlbaSimionesco, B. Frick, D. Morineau, J. Phys. Chem. C 2016, 120, 9245-9252.

[401] T. Bui, A. Phan, D. R. Cole, A. Striolo, J. Phys. Chem. C 2017, 121, 15675-15686.

[402] P. Ugliengo, M. Sodupe, F. Musso, I. J. Bush, R. Orlando, R. Dovesi, Adv. Mater. 2008, 20, 4579-4583.

[403] B. Coasne, P. Ugliengo, Langmuir 2012, 28, 11131-11141.

[404] M. Delle Piane, M. Corno, P. Ugliengo, Theor. Chem. Acc. 2016, 135, 1-10.

[405] A. Pedone, J. Bloino, V. Barone, J. Phys. Chem. C 2012, 116, 17807-17818.

[406] M. Delle Piane, M. Corno, A. Pedone, R. Dovesi, P. Ugliengo, J. Phys. Chem. C 2014, 118, 26737-26749.

[407] A. Cauvel, D. Brunel, F. DiRenzo, E. Garrone, B. Fubini, Langmuir 1997, 13, 2773-2778.

[408] M. F. Ottaviani, A. Galarneau, D. Desplantier-Giscard, F. Di Renzo, F. Fajula, Microporous Mesoporous Mater. 2001, 44-45, 1-8.

[409] E. Fois, A. Gamba, G. Tabacchi, Microporous Mesoporous Mater. 2008, 116, 718-722

[410] C. Allolio, F. Klameth, M. Vogel, D. Sebastiani, ChemPhysChem 2014, 15, 3955-3962.

[411] J. Geske, M. Vogel, Mol. Simul. 2017, 43, 13-18.

[412] M. Imperor-Clerc, P. Davidson, A. Davidson, J. Am. Chem. Soc 2000, 122, 11925-11933. 
[413] R. Ryoo, C. H. Ko, M. Kruk, V. Antochshuk, M. Jaroniec, J. Phys. Chem. B 2000, 104, 11465-11471.

[414] A. Galarneau, H. Cambon, F. Di Renzo, F. Fajula, Langmuir 2001, 17, 8328-8335.

[415] S. Naumov, R. Valiullin, J. Kärger, R. Pitchumani, M. O. Coppens, Microporous Mesoporous Mater. 2008, 110, 37-40.

[416] B. Rühle, M. Davies, T. Bein, C. Bräuchle, Zeitschrift für Naturforsch. B 2013, 68, 423-444.

[417] T. Lebold, L. A. Mühlstein, J. Blechinger, M. Riederer, H. Amentsch, R. Köhn, K. Peneva, K. Müllen, J. Michaelis, C. Bräuchte, T. Bein, Chem. Eur. J. 2009, 15, 1661-1672.

[418] B. Rühle, M. Davies, T. Lebold, C. Bräuchle, T. Bein, ACS Nano 2012, 6, 1948-1960.

[419] M. Davies, B. Rühle, C. Li, K. Müllen, T. Bein, C. Bräuchle, J. Phys. Chem. C 2014, 118, 24013-24024.

[420] C. Janiak, Angew. Chem. Int. Ed. Engl. 1997, 36, 1431-1434; Angew. Chem. 1997, 109, 1499-1502.

[421] J. Guo, Y. Xu, S. Jin, L. Chen, T. Kaji, Y. Honsho, M. A. Addicoat, J. Kim, A. Saeki, H. Ihee, S. Seki, S. Irle, M. Hiramoto, J. Gao, D. Jiang, Nat. Commun. 2013, 4, 2736.

[422] X. Feng, X. Ding, D. Jiang, Chem Soc Rev 2012, 41, 6010-22.

[423] X.-C. Huang, Y.-Y. Lin, J.-P. Zhang, X.-M. Chen, Angew. Chem. Int. Ed. 2006, 45, 1557-1559; Angew. Chem. 2006, 118, 1587-1589.

[424] M. Fischer, R. G. Bell, CrystEngComm 2014, 16, 1934.

[425] F. X. Coudert, ChemPhysChem 2017, 18, 2732-2738.

[426] R. Grau-Crespo, A. Aziz, A. W. Collins, R. Crespo-Otero, N. C. Hernández, L. M. Rodriguez-Albelo, A. R. Ruiz-Salvador, S. Calero, S. Hamad, Angew. Chem. Int. Ed. 2016, 55, 16012-16016; Angew. Chem. 2016, 128, 16246-16250.

[427] K. T. Butler, S. D. Worrall, C. D. Molloy, C. H. Hendon, M. P. Attfield, R. A. W. Dryfe, A. Walsh, J. Mater. Chem. C 2017, 5, 7726-7731.

[428] M. Eddaoudi, D. F. Sava, J. F. Eubank, K. Adil, V. Guillerm, Chem Soc. Rev. 2015, 44, 228-249.

[429] D. Farrusseng, S. Aguado, C. Pinel, Angew. Chem. Int. Ed. 2009, 48, 7502-7513; Angew. Chem. 2009, 121, 7638-7649.

[430] E. D. Bloch, W. L. Queen, M. R. Hudson, J. A. Mason, D. J. Xiao, L. J. Murray, R. Flacau, C. M. Brown, J. R. Long, Angew. Chem. Int. Ed. 2016, 55, 8605-8609; Angew. Chem. 2016, 128, 8747-8751.

[431] S. Bracco, F. Castiglioni, A. Comotti, S. Galli, M. Negroni, A. Maspero, P. Sozzani, Chem. Eur. J. 2017, 23, 11210-11215.

[432] S. J. Loeb, Chem. Commun. 2005, 0, 1511-1518.

[433] Q. Li, W. Zhang, O. Š. Miljanić, C. B. Knobler, J. F. Stoddart, O. M. Yaghi, Chem. Commun. 2010, 46, 380-382.

[434] Q. Li, C. H. Sue, S. Basu, A. K. Shveyd, W. Zhang, G. Barin, L. Fang, A. A. Sarjeant, J. F. Stoddart, O. M. Yaghi, Angew. Chem. Int. Ed. 2010, 49, 6751-6755; Angew. Chem. 2010, 122, 6903-6907.

[435] M. Mauro, R. Schuermann, C. Klaus, R. Prétôt, A. Hafner, P. Mercandelli, A. Sironi, L. De Cola, Angew. Chem. Int. Ed. 2010, 49, 1222-1226; Angew. Chem. 2010, 122, 1244-1248.

[436] M. O'Keeffe, M. A. Peskov, S. J. Ramsden, O. M. Yaghi, Acc. Chem. Res. 2008, 41, 1782-1789.

[437] K. Rissanen, Chem. Soc. Rev. 2017, 46, 2638-2648.

[438] Y. Inokuma, S. Yoshioka, J. Ariyoshi, T. Arai, Y. Hitora, K. Takada, S. Matsunaga, K. Rissanen, M. Fujita, Nature 2013, 495, 461.

[439] G. Brunet, D. A. Safin, K. Robeyns, G. A. Facey, I. Korobkov, Y. Filinchuk, M. Murugesu, Chem. Commun. 2017, 53, 5645-5648.

[440] A. Gasparotto, D. Barreca, C. Maccato, E. Tondello, Nanoscale 2012, 4, 2813.

[441] A. Mayoral, T. Carey, P. A. Anderson, A. Lubk, I. Diaz, Angew. Chem. Int. Ed. 2011, 50, 11230-11233; Angew. Chem. 2011, 123,
11426-11429.

[442] G. De Cremer, Y. Antoku, M. B. J. Roeffaers, M. Sliwa, J. Van Noyen, S. Smout, J. Hofkens, D. E. De Vos, B. F. Sels, T. Vosch, Angew. Chem. Int. Ed. 2008, 47, 2813-2816; Angew. Chem. 2008, 120, 2855-2858.

[443] A. Mayoral, T. Carey, P. A. Anderson, I. Diaz, Microporous Mesoporous Mater. 2013, 166, 117-122.

[444] G. De Cremer, B. F. Sels, J. I. Hotta, M. B. J. Roeffaers, E. Bartholomeeusen, E. Coutiño-Gonzalez, V. Valtchev, D. E. De Vos,

T. Vosch, J. Hofkens, Adv. Mater. 2010, 22, 957-960.

[445] K. Kennes, E. Coutino-Gonzalez, C. Martin, W. Baekelant, M. B. J. Roeffaers, M. Van der Auweraer, Adv. Funct. Mater. 2017, 27, 1606411.

[446] T. Yumura, A. Oda, H. Torigoe, A. Itadani, Y. Kuroda, T. Wakasugi, H. Kobayashi, J. Phys. Chem. C 2014, 118, 23874-23887.

[447] T. Yumura, M. Kumondai, Y. Kuroda, T. Wakasugi, H. Kobayashi, RSC Adv. 2017, 7, 4950-4959.

[448] C. E. Martinez-Nunez, M. Cortez-Valadez, Y. Delgado-Beleno, N. S. Flores-Lòpez, J. F. Romàn-Zamorano, J. Flores-Valenzuela, M. Flores-Acosta, J. Nanoparticle Res. 2017, 19, 31.

[449] N. C. Jeong, H. S. Kim, K. B. Yoon, J. Phys. Chem. C 2007, 111, 10298-10312.

[450] Herron N, Inorg. Chem. 1986, 25, 4714-4717.

[451] A. Corma, H. Garcia, Eur. J. Inorg. Chem. 2004, 2004, 1143-1164.

[452] L. Liu, U. Díaz, R. Arenal, G. Agostini, P. Concepción, A. Corma, Nat. Mater. 2016, 16, 132-138.

[453] T. Hirai, H. Okubo, I. Komasawa, J. Phys. Chem. B 1999, 103, 4228-4230.

[454] L. Pauling, Zeitschrift für Krist. 1930, 213-225.

[455] R. X. Fischer, W. H. Baur, Zeitschrift für Krist. 2009, 224, 185-197.

[456] M. Ganio, E. S. Pouyet, S. M. Webb, C. M. Schmidt Patterson, M. S Walton, Pure Appl. Chem. 2018, 90, 463-475.

[457] B. L. Sherriff, E. V Sokolova, J. Cramer, G. Kunath-Fandrei, C. J. Ger, L. A. Pautov, Am. Mineral. 1997, 82, 405-415.

[458] F. J. Ma, S. X. Liu, C. Y. Sun, D. D. Liang, G. J. Ren, F. Wei, Y. G. Chen, Z. M. Su, J. Am. Chem. Soc. 2011, 133, 4178-4181.

[459] M. Brandle, G. Calzaferri, Res. Chem. Intermed. 1994, 20, 783-806.

[460] A. Trave, F. Buda, A. Selloni, J. Phys. Chem. B 1998, 102, 15221527.

[461] F. Buda, A. Fasolino, Phys. Rev. B 1999, 60, 6131-6136.

[462] J. Antúnez-García, A. Posada-Amarillas, D. H. Galván, E. Smolentseva, V. Petranovskii, S. F. Moyado, RSC Adv. 2016, 6 , 79160-79165.

[463] L. Grajciar, J. Phys. Chem. C 2016, 120, 27050-27065.

[464] S. G. Chiodo, T. Mineva, J. Phys. Chem. C 2016, 120, 4471-4480.

[465] D. Brühwiler, R. Seifert, G. Calzaferri, J. Phys. Chem. B 1999, 103, 6397-6399.

[466] E. Coutino-Gonzalez, W. Baekelant, D. Grandjean, M. B. J. Roeffaers, E. Fron, M. S. Aghakhani, N. Bovet, M. Van der Auweraer, P. Lievens, T. Vosch, B. Sels, J. Hofkens, J. Mater Chem. C 2015, 3, 11857-11867.

[467] I. Tkach, A. Baldansuren, E. Kalabukhova, S. Lukin, A. Sitnikov, A. Tsvir, M. Ischenko, Y. Rosentzweig, E. Roduner, Appl. Magn. Reson. 2008, 35, 95-112.

[468] A. Baldansuren, R.-A. Eichel, E. Roduner, Phys. Chem. Chem. Phys. 2009, 11, 6664.

[469] A. Baldansuren, E. Roduner, Chem. Phys. Lett. 2009, 473, 135-137.

[470] A. Baldansuren, H. Dilger, R. A. Eichel, J. A. Van Bokhoven, E. Roduner, J. Phys. Chem. C 2009, 113, 19623-19632.

[471] E. Coutino-Gonzalez, M. B. J. Roeffaers, B. Dieu, G. De Cremer, S. 
Leyre, P. Hanselaer, W. Fyen, B. Sels, J. Hofkens, J. Phys. Chem. C 2013, 117, 6998-7004.

[472] O. Fenwick, E. Coutiño-Gonzalez, D. Grandjean, W. Baekelant, F. Richard, S. Bonacchi, D. De Vos, P. Lievens, M. Roeffaers, J. Hofkens, P. Samorì, Nat. Mater. 2016, 15, 1017-1022.

[473] G. De Cremer, E. Coutiño-Gonzalez, M. B. J. Roeffaers, D. E. De Vos, J. Hofkens, T. Vosch, B. F. Sels, ChemPhysChem 2010, 11, 1627-1631.

[474] T. Yumura, T. Nanba, H. Torigoe, Y. Kuroda, H. Kobayashi, Inorg. Chem. 2011, 50, 6533-6542.

[475] M. Meyer, A. Currao, G. Calzaferri, ChemPhysChem 2005, 6, 21672178.

[476] M. Meyer, C. Leiggener, G. Calzaferri, ChemPhysChem 2005, 6, 1071-1080.

[477] G. Calzaferri, Phys. Chem. Chem. Phys. 2017, 19, 10611-10621.

[478] J. Kecht, Z. Tahri, V. De Waele, M. Mostafavi, S. Mintova, T. Bein, Chem. Mater. 2006, 18, 3373-3380.

[479] E. Roduner, C. Jensen, J. Vanslageren, R. A. Rakoczy, O. Larlus, M. Hunger, Angew. Chem. Int. Ed. 2014, 53, 4318-4321; Angew. Chem. 2014, 126, 4406-4409.

[480] X. Liu, M. Bauer, H. Bertagnolli, E. Roduner, J. Van Slageren, F. Phillipp, Phys. Rev. Lett. 2006, 97, 253401.

[481] T. Schmauke, R.-A. Eichel, A. Schweiger, E. Roduner, Phys. Chem. Chem. Phys. 2003, 5, 3076.

[482] O. Terekhina, E. Roduner, J. Phys. Chem. C 2012, 116, 6973-6979.

[483] D. J. Moon, W. T. Lim, K. Seff, J. Phys. Chem. C 2016, 120, 1672216731.

[484] M. Zaarour, B. Dong, I. Naydenova, R. Retoux, S. Mintova, Microporous Mesoporous Mater. 2014, 189, 11-21.

[485] H. S. Kim, N. C. Jeong, K. B. Yoon, J. Am. Chem. Soc. 2011, 133, 1642-1645.

[486] H. S. Kim, N. C. Jeong, K. B. Yoon, Langmuir 2011, 27, 1467814688.

[487] D. Hu, Y. Zhang, J. Lin, Y. Hou, D. Li, T. Wu, Dalt. Trans. 2017, 46, 3929-3933.

[488] F. J. Brieler, P. Grundmann, M. Fröba, L. Chen, P. J. Klar, W. Heimbrodt, H. A. K. Von Nidda, T. Kurz, A. Loidl, J. Am. Chem. Soc 2004, 126, 797-807.

[489] S. Alam, C. Anand, K. Ariga, T. Mori, A. Vinu, Angew. Chem. Int. Ed. 2009, 48, 7358-7361; Angew. Chem. 2009, 121, 7494-7497.

[490] J. Burschka, N. Pellet, S.-J. Moon, R. Humphry-Baker, P. Gao, M. K. Nazeeruddin, M. Grätzel, Nature 2013, 499, 316-319.

[491] F. Bertolotti, L. Protesescu, M. V. Kovalenko, S. Yakunin, A. Cervellino, S. J. L. Billinge, M. W. Terban, J. S. Pedersen, N. Masciocchi, A. Guagliardi, ACS Nano 2017, 11, 3819-3831.

[492] D. N. Dirin, L. Protesescu, D. Trummer, I. V. Kochetygov, S. Yakunin, F. Krumeich, N. P. Stadie, M. V. Kovalenko, Nano Lett. 2016, 16, 5866-5874

[493] J. Aguilera-Sigalat, D. Bradshaw, Coord. Chem. Rev. 2015, 307, 267-291.

[494] C. R. Kim, T. Uemura, S. Kitagawa, Chem. Soc. Rev. 2016, 45, 3828-3845.

[495] G. Lu, S. Li, Z. Guo, O. K. Farha, B. G. Hauser, X. Qi, Y. Wang, X. Wang, S. Han, X. Liu, J. S. Duchene, H. Zhang, Q. Zhang, X. Chen, J. Ma, S. C. J. Loo, W. D. Wei, Y. Yang, et al., Nat. Chem. 2012, 4, 310-316.

[496] J. R. Avila, A. W. Peters, Z. Li, M. A. Ortuño, A. B. F. Martinson, C. J. Cramer, J. T. Hupp, O. K. Farha, Dalt. Trans. 2017, 46, 57905795 .

[497] T. Islamoglu, S. Goswami, Z. Li, A. J. Howarth, O. K. Farha, J. T.
Hupp, Acc. Chem. Res. 2017, 50, 805-813.

[498] J.-J. Liu, Y.-F. Guan, L. Li, Y. Chen, W.-X. Dai, C.-C. Huang, M.-J. Lin, Chem. Commun. 2017, 53, 4481-4484.

[499] E. Meneghinello, A. Martucci, A. Alberti, F. Di Renzo, Microporous Mesoporous Mater. 1999, 30, 89-94.

[500] A. Godelitsas, T. Armbruster, Microporous Mesoporous Mater. 2003, 61, 3-24.

[501] A. Godelitsas, D. Charistos, A. Tsipis, C. Tsipis, A. Filippidis, C. Triantafyllidis, G. Manos, D. Siapkas, Chemistry 2001, 7, 3705-21.

[502] G. D. Gatta, P. Lotti, F. Nestola, D. Pasqual, Microporous Mesoporous Mater. 2012, 163, 259-269.

[503] T. Frising, P. Leflaive, Microporous Mesoporous Mater. 2008, 114 27-63.

[504] E. Passaglia, R. A. Sheppard, Rev. Mineral. Geochemistry 2001, 45

[505] L. Campana, A. Selloni, J. Weber, A. Goursot, J. Phys. Chem. B 1997, 101, 9932-9939.

[506] L. Bernasconi, E. Fois, A. Selloni, J. Chem. Phys. 1999, 24, 90489055.

[507] A. R. Ruiz-Salvador, N. Almora-Barrios, A. Gomez, D. W. Lewis, A. Gomez, D. W. Lewis, Phys. Chem. Chem. Phys. 2007, 9, 521-532.

[508] S. R. G. Balestra, S. Hamad, A. R. Ruiz-Salvador, V.

Domínguez-García, P. J. Merkling, D. Dubbeldam, S. Calero, Chem. Mater. 2015, 27, 5657-5667.

[509] I. A. Bryukhanov, A. A. Rybakov, A. V Larin, D. N. Trubnikov, D. P. Vercauteren, J. Mol. Model. 2017, 23, 68.

[510] X.-J. Hou, H. Li, P. He, S. Li, Mol. Simul. 2017, 43, 926-934.

[511] P. Demontis, J. Gulín-Gonzalez, G. B. Suffritti, J. Phys. Chem. B 2006, 110, 7513-8.

[512] K. S. Smirnov, D. Bougeard, J. Phys. Condens. Matter 2010, 22, 284115

[513] P. Demontis, J. Gulín-González, M. Masia, G. B. Suffritti, J. Phys. Condens. Matter 2010, 22, 284106.

[514] P. Demontis, J. Gulin-González, H. Jobic, G. B. Suffritti, J. Phys. Chem. C 2010, 114, 18612-18621.

[515] P. Demontis, H. Jobic, M. A. Gonzalez, G. B. Suffritti, J. Phys. Chem. C 2009, 113, 12373-12379.

[516] E. Fois, A. Gamba, G. Tabacchi, F. Trudu, Stud. Surf. Sci. Catal. 2008, 174, 751-754.

[517] M. Fischer, Zeitschrift für Krist. - Cryst. Mater. 2015, 230, 325-336.

[518] P. Gómez-Álvarez, S. Calero, CrystEngComm 2015, 17, 412-421.

[519] E. Fois, A. Gamba, A. Tilocca, J. Phys. Chem. B 2002, 106, 48064812.

[520] J. Hunger, I. A. Beta, H. Böhlig, C. Ling, H. Jobic, B. Hunger, J. Phys. Chem. B 2006, 110, 342-353.

[521] A. Di Lella, N. Desbiens, A. Boutin, I. Demachy, P. Ungerer, J.-P. Bellat, A. H. Fuchs, Phys. Chem. Chem. Phys. 2006, 8, 5396.

[522] P. Demontis, G. Stara, G. B. Suffritti, J. Phys. Chem. B 2003, 107, 4426-4436.

[523] T. Zhou, P. Bai, J. I. Siepmann, A. E. Clark, J. Phys. Chem. C 2017, 121, 22015-22024.

[524] P. Demontis, J. Gulìn-Gonzàlez, H. Jobic, M. Masia, R. Sale, G. B. Suffritti, ACS Nano 2008, 2, 1603-1614.

[525] N. Floquet, J. P. Coulomb, N. Dufau, G. Andre, C. R. M. C. Cnrs, C. De Luminy, M. Cedex, J. Phys. Chem. B 2004, 108, 13107-13115.

[526] F.-X. Coudert, R. Vuilleumier, A. Boutin, ChemPhysChem 2006, 7, 2464-2467.

[527] F.-X. Coudert, F. Cailliez, R. Vuilleumier, A. H. Fuchs, A. Boutin, Faraday Discuss. 2009, 141, 377-398.

[528] E. Fois, A. Gamba, G. Tabacchi, R. Arletti, S. Quartieri, G. Vezzalini, Am. Mineral. 2005, 90, 28-35. 
[529] A. Alberti, G. Cruciani, E. Galli, S. Merlino, R. Millini, S. Quartieri, G. Vezzalini, S. Zanardi, J. Phys. Chem. B 2002, 106, 10277-10284.

[530] H. Effenberger, G. Giester, W. Krause, H.-J. Bernhardt, Am. Mineral. 1998, 83, 607-617.

[531] E. Galli, S. Quartieri, G. Vezalini, A. Alberti, Eur. J. Mineral. 1995, 7, 1029-1032.

[532] A. Martucci, A. Alberti, M. de Lourdes Guzman-Castillo, F. Di Renzo, F. Fajula, Microporous Mesoporous Mater. 2003, 63, 33-42.

[533] R. C. Boggs, D. G. Howard, J. V. Smith, G. L. Klein, Am. Mineral. 1993, 78.

[534] G. D. Gatta, P. Lotti, G. Tabacchi, Phys. Chem. Miner. 2018, 45, 115-138.

[535] G. D. Gatta, Zeitschrift für Krist. 2008, 223, 160-170.

[536] R. Arletti, O. Ferro, S. Quartieri, A. Sani, G. Tabacchi, G. Vezzalini, Am. Mineral. 2003, 88, 1416-1422.

[537] G. D. Gatta, Eur. J. Mineral. 2005, 17, 411-422.

[538] A. Sartbaeva, S. A. Wells, M. M. J. Treacy, M. F. Thorpe, Nat. Mater 2006, 5, 962-965.

[539] A. Sartbaeva, G. D. Gatta, S. A. Wells, Europhys. Lett. 2008, 83, 26002.

[540] P. Ballone, S. Quartieri, A. Sani, G. Vezzalini, Am. Mineral. 2002, 87, 1194-1206.

[541] E. Fois, A. Gamba, G. Tabacchi, S. Quartieri, R. Arletti, G. Vezzalini, Stud. Surf. Sci. Catal. 2005, 155, 271-280.

[542] J. S. Tse, D. D. Klug, J. A. Ripmeester, S. Desgreniers, K. Lagarec, Nature 1994, 369, 724-727.

[543] K. Ståhl, Å. Kvick, S. Ghose, Zeolites 1989, 9, 303-311.

[544] S. Quartieri, A. Sani, G. Vezzalini, E. Galli, E. Fois, A. Gamba, G. Tabacchi, Microporous Mesoporous Mater. 1999, 30, 77-87.

[545] E. Fois, G. Tabacchi, S. Quartieri, G. Vezzalini, J. Chem. Phys. 1999, 111, 355-359.

[546] C. Ceriani, E. Fois, A. Gamba, G. Tabacchi, O. Ferro, S. Quartieri, G. Vezzalini, Am. Mineral. 2004, 89, 102-109.

[547] O. Ferro, S. Quartieri, G. Vezzalini, E. Fois, A. Gamba, G. Tabacchi, Am. Mineral. 2002, 87, 1415-1425.

[548] P. Comodi, G. D. Gatta, P. F. Zanazzi, Eur. J. Mineral. 2003, 15 247-255.

[549] E. Fois, A. Gamba, G. Tabacchi, O. Ferro, S. Quartieri, G. Vezzalini, Stud. Surf. Sci. Catal. 2002, 142 B, 1877-1884.

[550] Y. V. Seryotkin, Microporous Mesoporous Mater. 2016, 226, 415423.

[551] P. Demontis, G. Stara, G. B. Suffritti, J. Chem. Phys. 2004, 120, 9233-9244.

[552] A. V. Larin, D. N. Trubnikov, D. P. Vercauteren, Int. J. Quantum Chem. 2003, 92, 71-84.

[553] B. A. Kolesov, J. Struct. Chem. 2006, 47, 21-34.

[554] B. A. Kolesov, C. A. Geiger, Am. Mineral. 2002, 87, 1426-1431.

[555] K. Larsson, J. Tegenfeldt, Å. Kvick, J. Phys. Chem. Solids 1989, 50, 107-110.

[556] P. Norby, A. N. Christensen, I. G. K. Andersen, 1986, 40A, 500-506.

[557] K. Larsson, J. Tegenfeldt, K. Hermansson, J. Chem. Soc. Faraday Trans 1991, 87, 1193-1200.

[558] E. Fois, A. Gamba, G. Tabacchi, S. Quartieri, G. Vezzalini, J. Phys. Chem. B 2001, 105, 3012-3016.

[559] E. Fois, A. Gamba, G. Tabacchi, S. Quartieri, G. Vezzalini, Phys. Chem. Chem. Phys. 2001, 3, 4158-4163.

[560] P. Demontis, G. Stara, G. B. Suffritti, Microporous Mesoporous Mater. 2005, 86, 166-175.

[561] T. Wen, W. Zhang, X. Hu, L. He, H. Li, ChemPlusChem 2013, 78, 438-442.
[562] R. Q. Albuquerque, Z. Popović, L. De Cola, G. Calzaferri, ChemPhysChem 2006, 7, 1050-1053.

[563] G. Calzaferri, H. Li, D. Brühwiler, Chem. Eur. J. 2008, 14, 74427449 .

[564] A. Devaux, G. Calzaferri, Int. J. Photoenergy 2009, 2009, 1-9.

[565] S. Hashimoto, J. Phys. Chem. Lett. 2011, 2, 509-519.

[566] L. Gartzia-Rivero, J. Bañuelos, I. López-Arbeloa, Int. Rev. Phys Chem. 2015, 34, 515-556.

[567] T. Hertzsch, F. Budde, E. Weber, J. Hulliger, Angew. Chem. Int. Ed. 2002, 41, 2281-2284; Angew. Chem. 2002, 114, 2385-2388.

[568] Y. Q. Hu, M. Q. Li, Y. Wang, T. Zhang, P. Q. Liao, Z. Zheng, X. M. Chen, Y. Z. Zheng, Chem. Eur. J. 2017, 23, 8409-8413.

[569] G. Wirnsberger, H. P. Fritzer, A. Popitsch, G. van de Goor, P. Behrens, Angew. Chem. Int. Ed. Engl. 1996, 35, 2777-2779; Angew. Chem. 1996, 108, 2951 -2953.

[570] G. Flachenecker, A. Materny, J. Chem. Phys. 2004, 120, 56745690.

[571] M. Kocirik, J. Kornatowski, V. Masařík, P. Novák, A. Zikánová, J. Maixner, Microporous Mesoporous Mater. 1998, 23, 295-308.

[572] F. Y. Jiang, R. C. Liu, J. Phys. Chem. Solids 2007, 68, 1552-1555.

[573] J. T. Ye, Z. K. Tang, G. G. Siu, Appl. Phys. Lett. 2006, 88, 73114.

[574] W. Guo, D. Wang, J. Hu, Z. K. Tang, S. Du, Appl. Phys. Lett. 2011, 98, 43105.

[575] J. P. Zhai, I. L. Li, S. C. Ruan, H. F. Lee, Z. K. Tang, Appl. Phys. Lett. 2008, 92, 43117.

[576] M. Golecki, N. Beyer, G. Steinfeld, V. Lozan, S. Voitekhovich, M. Sehabi, J. Möllmer, H. J. Krüger, B. Kersting, Angew. Chem. Int. Ed 2014, 53, 9949-9952; Angew. Chem. 2014, 126,10107-10111.

[577] G. Massasso, J. Long, C. Guerin, A. Grandjean, B. Onida, Y. Guari, J. Larionova, G. Maurin, S. Devautour-Vinot, J. Phys. Chem. C 2015, 119, 9395-9401.

[578] G. Massasso, M. Rodríguez-Castillo, J. Long, J. Haines, S. Devautour-Vinot, G. Maurin, A. Grandjean, B. Onida, B. Donnadieu, J. Larionova, C. Guérin, Y. Guari, Dalt. Trans. 2015, 44, 1935719369.

[579] C. Falaise, C. Volkringer, J. Facqueur, T. Bousquet, L. Gasnot, T. Loiseau, Chem. Commun. 2013, 49, 10320-10322.

[580] G. Brunet, D. A. Safin, M. Z. Aghaji, K. Robeyns, I. Korobkov, T. K. Woo, M. Murugesu, Chem. Sci. 2017, 8, 3171-3177.

[581] S.-S. Zhao, L. Chen, X. Zheng, L. Wang, Z. Xie, Chem. Asian J. 2017, 12, 615-620

[582] Y. He, J. Zhang, L. Lei, W. Kong, Angew. Chem. Int. Ed. 2017, 56, 3541-3545; Angew. Chem. 2017, 129, 3595-3599.

[583] J. M. Hu, J. P. Zhai, F. M. Wu, Z. K. Tang, J. Phys. Chem. B 2010, $114,16481-16486$.

[584] J. Hu, D. Wang, W. Guo, S. Du, Z. K. Tang, J. Phys. Chem. C 2012 116, 4423-4430

[585] S. Chen, M. Yao, Y. Yuan, F. Ma, Z. Liu, B. Li, R. Liu, Q. Li, B. Zou, T. Cui, B. Liu, J. Raman Spectrosc. 2015, 46, 400-405.

[586] Z. Liu, M. Yao, Y. Yuan, S. Chen, R. Liu, S. Lu, B. Zou, T. Cui, B. Liu, J. Raman Spectrosc. 2015, 46, 413-417.

[587] S. Chen, Y. Yuan, X. Yang, M. Du, Z. Liu, R. Liu, B. Li, B. Zou, T. Cui, B. Liu, Microporous Mesoporous Mater. 2016, 221, 76-80.

[588] S. Chen, M. Yao, Y. Yuan, F. Ma, Z. Liu, R. Liu, W. Cui, X. Yang, B. Liu, B. Zou, T. Cui, B. Liu, Phys. Chem. Chem. Phys. 2014, 16, 8301-9.

[589] M. Yao, T. Wang, Z. Yao, D. Duan, S. Chen, Z. Liu, R. Liu, S. Lu, Y. Yuan, B. Zou, T. Cui, B. Liu, J. Phys. Chem. C 2013, 117, 2505225058

[590] Z. Liu, Z. Yao, M. Yao, J. Lv, S. Chen, Q. Li, H. Lv, T. Wang, S. Lu, 
R. Liu, B. Liu, J. Liu, Z. Chen, B. Zou, T. Cui, B. Liu, J. Chem. Phys. 2016, 145, 124319

[591] E. Fois, A. Gamba, E. Spano, G. Tabacchi, J. Mol. Struct. 2003, 644, 55-66.

[592] Y. Yuan, M. Yao, S. Chen, S. Liu, X. Yang, W. Zhang, Z. Yao, R. Liu, B. Liu, B. Liu, Nanoscale 2016, 8, 1456-1461.

[593] S. Megelski, A. Lieb, M. Pauchard, A. Drechsler, S. Glaus, C. Debus, A. J. Meixner, G. Calzaferri, J. Phys. Chem. B 2001, 105, 25-35.

[594] S. Huber, A. Z. Ruiz, H. Li, G. Patrinoiu, C. Botta, G. Calzaferri, Inorganica Chim. Acta 2007, 360, 869-875.

[595] E. Fois, G. Tabacchi, G. Calzaferri, J. Phys. Chem. C 2012, 116 16784-16799.

[596] E. Fois, G. Tabacchi, A. Devaux, P. Belser, D. Brühwiler, G. Calzaferri, Langmuir 2013, 29, 9188-9198.

[597] N. Ozaki, H. Sakamoto, T. Nishihara, T. Fujimori, Y. Hijikata, R. Kimura, S. Irle, K. Itami, Angew. Chem. Int. Ed. 2017, 56, 1119611202; Angew. Chem. 2017, 129, 11348-11354.

[598] V. E. Bondybey, C. Fletcher, J. Chem. Phys. 1976, 64, 3615-3620.

[599] E. Fois, A. Gamba, C. Medici, G. Tabacchi, ChemPhysChem 2005, 6, 1917-1922.

[600] I. Frank, J. Hutter, D. Marx, M. Parrinello, J. Chem. Phys. 1998, 108, 4060.

[601] D. L. Dexter, J. Chem. Phys. 1953, 21, 836-850.

[602] B. Ye, M. L. Trudeau, D. M. Antonelli, Adv. Mater. 2001, 13, 561565.

[603] T. Itoh, K. Yano, T. Kajino, S. Itoh, Y. Shibata, H. Mino, R. Miyamoto, Y. Inada, S. Iwai, Y. Fukushima, J. Phys. Chem. B 2004, 108, 13683-13687.

[604] I. Oda, K. Hirata, S. Watanabe, Y. Shibata, T. Kajino, Y. Fukushima, S. Iwai, S. Itoh, J. Phys. Chem. B 2006, 110, 1114-1120.

[605] H. Ikemoto, S. Tubasum, T. Pullerits, J. Ulstrup, Q. Chi, J. Phys. Chem. C 2013, 117, 2868-2878.

[606] M. B. Majewski, A. J. Howarth, P. Li, M. R. Wasielewski, J. T. Hupp, O. K. Farha, CrystEngComm 2017, 19, 4082-4091.

[607] G. Tabacchi, G. Calzaferri, E. Fois, Chem. Commun. 2016, 52, 11195-11198.

[608] R. M. Barrer, E. A. D. White, J. Chem. Soc. 1951, 0, 1267.

[609] G. Tabacchi, S. Silvi, M. Venturi, A. Credi, E. Fois, ChemPhysChem 2016, 17, 1913-1919.

[610] S. A. Moggach, T. D. Bennett, A. K. Cheetham, Angew. Chem. Int Ed. 2009, 48, 7087-7089; Angew. Chem. 2009, 121, 7221-7223.

[611] E. Haldoupis, T. Watanabe, S. Nair, D. S. Sholl, ChemPhysChem 2012, 13, 3449-3452

[612] G. Calzaferri, N. Gfeller, J. Phys. Chem. 1992, 96, 3428-3435.

[613] F. Binder, G. Calzaferri, N. Gfeller, Sol. Energy Mater. Sol. Cells 1995, 38, 175-186.

[614] M. Pauchard, S. Huber, R. Méallet-Renault, H. Maas, R. Pansu, G. Calzaferri, Angew. Chem. Int. Ed. 2001, 40, 2839-2842; Angew. Chem. 2001, 113, 2921-2924.

[615] G. Calzaferri, M. Pauchard, H. Maas, S. Huber, A. Khatyr, T. Schaafsma, J. Mater. Chem. 2002, 12, 1-13.

[616] D. Brühwiler, G. Calzaferri, Comptes Rendus Chim. 2005, 8, 391398.

[617] C. Minkowski, G. Calzaferri, Angew. Chem. Int. Ed. 2005, 44, 53255329; Angew. Chem. 2005, 117, 5459-5463.

[618] A. Devaux, G. Calzaferri, P. Belser, P. Cao, D. Brühwiler, A. Kunzmann, Chem. Mater. 2014, 26, 6878-6885.

[619] J. El-Gindi, K. Benson, L. De Cola, H. J. Galla, N. Seda Kehr, Angew. Chem. Int. Ed. 2012, 51, 3716-3720; Angew. Chem. 2012, 124, 3776-3780.
E. A. Prasetyanto, P. Manini, A. Napolitano, O. Crescenzi, M. d'Ischia, L. De Cola, Chem. Eur. J. 2014, 20, 1597-1601.

[621] A. Bertucci, H. Lülf, D. Septiadi, A. Manicardi, R. Corradini, L. De Cola, Adv. Healthc. Mater. 2014, 3, 1812-1817.

[622] B. Ergün, L. De Cola, H.-J. Galla, N. S. Kehr, Adv. Healthc. Mater. 2016, 5, 1588-1592.

[623] A. Greco, L. Maggini, L. De Cola, R. De Marco, L. Gentilucci, Bioconjug. Chem. 2015, 26, 1873-1878.

[624] M. Becker, L. De Cola, A. Studer, Chem. Commun. 2011, 47, 3392.

[625] D. Kim, H. S. Kim, Microporous Mesoporous Mater. 2017, 243, 6975.

[626] V. Martínez-Martínez, R. García, L. Gómez-Hortigüela, J. PérezPariente, I. López-Arbeloa, Chem. Eur. J. 2013, 19, 9859-9865.

[627] R. Sola-Llano, V. Martínez-Martínez, Y. Fujita, L. Gómez-Hortigüela A. Alfayate, H. Uji-i, E. Fron, J. Pérez-Pariente, I. López-Arbeloa, Chem. Eur. J. 2016, 22, 15700-15711.

[628] V. Martínez-Martínez, R. García, L. Gómez-Hortigüela, R. Sola Llano, J. Pérez-Pariente, I. López-Arbeloa, ACS Photonics 2014, 1, 205-211.

[629] D. Lencione, M. H. Gehlen, L. N. Trujillo, R. C. F. Leitao, R. Q. Albuquerque, Photochem. Photobiol. Sci. 2016, 15, 398-404.

[630] M. Łukarska, A. Jankowska, J. Gapiński, S. Valable, C. Anfray, B. Menard, S. Mintova, S. Kowalak, New J. Chem. 2017, 41, 99699976.

[631] S. C. Mohan, R. Vijay Solomon, P. Venuvanalingam, K Jothivenkatachalam, New J. Chem. 2017, 41, 9505-9512.

[632] H. Awala, E. Leite, L. Saint-Marcel, G. Clet, R. Retoux, I. Naydenova, S. Mintova, New J. Chem. 2016, 40, 4277-4284.

[633] B. Onida, B. Bonelli, M. Lucco-Borlera, L. Flora, C. Otero Areán, E. Garrone, Stud. Surf. Sci. Catal. 2001, 135, 364.

[634] F. Cucinotta, F. Carniato, A. Devaux, L. De Cola, L. Marchese, Chem. Eur. J. 2012, 18, 15310-15315.

[635] B. Onida, B. Bonelli, L. Flora, F. Geobaldo, C. O. Arean, E. Garrone, Chem. Commun. 2001, 2216-2217.

[636] F. Cucinotta, B. P. Jarman, C. Caplan, S. J. Cooper, H. J. Riggs, J. Martinelli, K. Djanashvili, E. La Mazza, F. Puntoriero, ChemPhotoChem 2017, DOI 10.1002/cptc.201700144

[637] P. Kittikhunnatham, B. Som, V. Rassolov, M. Stolte, F. Würthner, L. S. Shimizu, A. B. Greytak, J. Phys. Chem. C 2017, 121, 18102 18109.

[638] W. P. Lustig, S. Mukherjee, N. D. Rudd, A. V. Desai, J. Li, S. K. Ghosh, Chem. Soc. Rev. 2017, 46, 3242-3285.

[639] M. C. So, G. P. Wiederrecht, J. E. Mondloch, J. T. Hupp, O. K. Farha, Chem. Commun. 2015, 51, 3501-3510.

[640] R. Medishetty, J. K. Zaręba, D. Mayer, M. Samoć, R. A. Fischer, Chem. Soc. Rev. 2017, 46, 4976-5004.

[641] M. Handke, T. Adachi, C. Hu, M. D. Ward, Angew. Chem. Int. Ed. 2017, 56, 14003-14006; Angew. Chem. 2017, 129, 14191-14194.

[642] T. H. Noh, J. Jang, W. Hong, H. Lee, O.-S. Jung, Chem. Commun. 2014, 50, 7451-7454.

[643] T. H. Noh, H. Lee, J. Jang, O. S. Jung, Angew. Chem. Int. Ed. 2015 54, 9284-9288; Angew. Chem. 2015, 127, 9416-9420.

[644] H. He, E. Ma, Y. Cui, J. Yu, Y. Yang, T. Song, C.-D. Wu, X. Chen, B. Chen, G. Qian, Nat. Commun. 2016, 7, 11087.

[645] H. Dong, C. Zhang, Y. S. Zhao, J. Mater. Chem. C 2017, 5, 56005609.

[646] H. He, E. Ma, J. Yu, Y. Cui, Y. Lin, Y. Yang, X. Chen, B. Chen, G. Qian, Adv. Opt. Mater. 2017, 5, 1601040.

[647] X.-Y. Liu, F. Zhang, T.-W. Goh, Y. Li, Y.-C. Shao, L. Luo, W. Huang Y.-T. Long, L.-Y. Chou, C.-K. Tsung, Angew. Chem. Int. Ed. 2018, 
57, 2110-2114; Angew. Chem. 2018, 130, 2132-2136.

[648] S. Megelski, G. Calzaferri, Adv. Funtional Mater. 2001, 11, 277-286.

[649] L. Gartzia-Rivero, J. Bañuelos, U. Izquierdo, V. L. Barrio, K. Bizkarra, J. F. Cambra, I. López-Arbeloa, Part. Part. Syst. Charact. 2014, 31, 110-120.

[650] H. S. Cho, A. R. Hill, M. Cho, K. Miyasaka, K. Jeong, M. W. Anderson, J. K. Kang, O. Terasaki, Cryst. Growth Des. 2017, 17, 4516-4521.

[651] Z. Popović, M. Busby, S. Huber, G. Calzaferri, L. De Cola, Angew. Chem. Int. Ed. 2007, 46, 8898-8902; Angew. Chem. 2007, 119, 9056-9060.

[652] S. Hashimoto, K. Samata, T. Shoji, N. Taira, T. Tomita, S. Matsuo, Microporous Mesoporous Mater. 2009, 117, 220-227.

[653] G. Calzaferri, R. Méallet-Renault, D. Brühwiler, R. Pansu, I. Dolamic, T. Dienel, P. Adler, H. Li, A. Kunzmann, ChemPhysChem 2011, 12, 580-594.

[654] Z. Popović, M. Otter, G. Calzaferri, L. De Cola, Angew. Chem. Int. Ed. 2007, 46, 6188-6191; Angew. Chem. 2007, 119, 6301-6304.

[655] C. A. Strassert, M. Otter, R. Q. Albuquerque, A. Hone, Y. Vida, B. Maier, L. De Cola, Angew. Chem. Int. Ed. 2009, 48, 7928-7931; Angew. Chem. 2009, 121, 8070-8073.

[656] H. van Koningsveld, J. H. Koegler, Microporous Mater. 1997, 9, 7181.

[657] B. Hennessy, S. Megelski, C. Marcolli, V. Shklover, C. Baerlocher, G. Calzaferri, J. Phys. Chem. B 1999, 103, 3340-3351.

[658] R. Hoppe, G. Schulz-Ekloff, D. Wöhrle, C. Kirschhock, H. Fuess, L. Uytterhoeven, R. Schoonheydt, Adv. Mater. 1995, 7, 61-64.

[659] P. Simoncic, T. Armbruster, P. Pattison, J. Phys. Chem. B 2004 108, 17352-17360.

[660] L. Gigli, R. Arletti, G. Tabacchi, E. Fois, J. G. Vitillo, G. Martra, G. Agostini, S. Quartieri, G. Vezzalini, J. Phys. Chem. C 2014, 118, 15732-15743.

[661] L. Gigli, R. Arletti, J. G. Vitillo, G. Alberto, G. Martra, A. Devaux, G. Vezzalini, J. Phys. Chem. C 2015, 119, 16156-16165.

[662] P. Simoncic, T. Armbruster, Microporous Mesoporous Mater. 2005, 81, 87-95

[663] C. Dejoie, P. Martinetto, N. Tamura, M. Kunz, F. Porcher, P. Bordat, R. Brown, E. Dooryhée, M. Anne, L. B. McCusker, J. Phys. Chem. C 2014, 118, 28032-28042.

[664] L. Gigli, R. Arletti, E. Fois, G. Tabacchi, S. Quartieri, V. Dmitriev, G. Vezzalini, Crystals 2018, 8, 79.

[665] P. Cao, O. Khorev, A. Devaux, L. Sägesser, A. Kunzmann, A. Ecker, R. Häner, D. Brühwiler, G. Calzaferri, P. Belser, Chem. Eur. J. 2016, 22, 4046-4060.

[666] H. Maas, G. Calzaferri, Angew. Chem. Int. Ed. 2002, 41, 22842288; Angew. Chem. 2002, 114, 2389-2392.

[667] S. Huber, G. Calzaferri, Angew. Chem. Int. Ed. 2004, 43, 67386742; Angew. Chem. 2004, 116, 6906-6910.

[668] F. Würthner, Chem. Commun. 2004, 1564-1579.

[669] F. Würthner, C. R. Saha-Möller, B. Fimmel, S. Ogi, P. Leowanawat, D. Schmidt, Chem. Rev. 2015, 116, 962-1052.

[670] Y. Wang, H. Li, Y. Feng, H. Zhang, G. Calzaferri, T. Ren, Angew. Chem. Int. Ed. 2010, 49, 1434-1438; Angew. Chem. 2010, 122 , 1476-1480.

[671] A. Z. Ruiz, H. Li, G. Calzaferri, Angew. Chem. Int. Ed. 2006, 45, 5282-5287; Angew. Chem. 2006, 118, 5408-5413.

[672] N. S. Kehr, B. Ergün, H. Lülf, L. De Cola, Adv. Mater. 2014, 26 , 3248-3252.

[673] S. Fibikar, G. Luppi, V. Martínez-Junza, M. Clemente-León, L. De Cola, ChemPlusChem 2015, 80, 62-67.
[674] L.-Q. Dieu, A. Devaux, I. López-Duarte, M. Victoria Martínez-Díaz, D. Brühwiler, G. Calzaferri, T. Torres, Chem. Commun. 2008, 0, 1187-1189.

[675] M. Gruner, V. Siozios, B. Hagenhoff, D. Breitenstein, C. A. Strassert Photochem. Photobiol. 2013, 89, 1406-1412.

[676] I. Lõpez-Duarte, Le-Quyenha Dieu, I. Dolamic, M. V. Martínez-Díaz, T. Torres, G. Calzaferri, D. Brühwiler, Chem. Eur. J. 2011, 17, 1855-1862.

[677] R. Q. Albuquerque, J. Kühni, P. Belser, L. De Cola ChemPhysChem 2010, 11, 575-578.

[678] F. Cucinotta, A. Guenet, C. Bizzarri, W. Mroz, C. Botta, B. MilianMedina, J. Gierschner, L. De Cola, ChemPluschem 2014, 79, 45-57.

[679] J. M. Beierle, R. Roswanda, P. M. Erne, A. C. Coleman, W. R. Browne, B. L. Feringa, Part. Part. Syst. Charact. 2013, 30, 273-279.

[680] A. Devaux, C. Minkowski, G. Calzaferri, Chem. Eur. J. 2004, 10 2391-2408.

[681] B. Bussemer, D. Munsel, H. Wünscher, G. J. Mohr, U. W. Grummt, J. Phys. Chem. B 2007, 111, 8-15.

[682] S. Hashimoto, M. Hagiri, N. Matsubara, S. Tobita, Phys. Chem. Chem. Phys. 2001, 3, 5043-5051.

[683] W. Insuwan, K. Rangsriwatananon, J. Porous Mater. 2014, 21, 345 354.

[684] C. Minkowski, R. Pansu, M. Takano, G. Calzaferri, Adv. Funct. Mater. 2006, 16, 273-285.

[685] M. Busby, A. Devaux, C. Blum, V. Subramaniam, G. Calzaferri, L. De Cola, J. Phys. Chem. C 2011, 115, 5974-5988.

[686] A. Devaux, G. Calzaferri, I. Miletto, P. Cao, P. Belser, D. Brühwiler, O. Khorev, R. Häner, A. Kunzmann, J. Phys. Chem. C 2013, 117, 23034-23047.

[687] E. Fois, G. Tabacchi, G. Calzaferri, J. Phys. Chem. C 2010, 114, 10572-10579.

[688] X. Zhou, T. A. Wesolowski, G. Tabacchi, E. Fois, G. Calzaferri, A. Devaux, Phys. Chem. Chem. Phys. 2013, 15, 159-167.

[689] S. Deore, P. Simoncic, A. Navrotsky, Microporous Mesoporous Mater. 2008, 109, 342-349.

[690] S. Doungmanee, T. Siritanon, W. Insuwan, S. Jungsuttiwong, K. Rangsriwatananon, J. Porous Mater. 2018, https://doi.org/10.1007/s10934-017-0550-7.

[691] J. Tsuwi, R. Berger, G. Labat, G. Couderc, N. R. Behrnd, P. Ottiger, F. Cucinotta, K. Schürmann, M. Bertoni, L. Viani, J. Gierschner, J. Cornil, A. Prodi-Schwab, L. De Cola, M. Wübbenhorst, J. Hulliger, J. Phys. Chem. A 2010, 114, 6956-6963.

[692] L. Viani, A. Minoia, J. Cornil, D. Beljonne, H. J. Egelhaaf, J. Gierschner, J. Phys. Chem. C 2016, 120, 27192-27199.

[693] L. Gartzia-Rivero, J. Bañuelos-Prieto, V. Martínez-Martínez, I. López Arbeloa, ChemPluschem 2012, 77, 61-70.

[694] W. Insuwan, S. Jungsuttiwong, K. Rangsriwatananon, J. Lumin 2015, 161, 31-36.

[695] W. Insuwan, K. Rangsriwatananon, J. Meeprasert, S. Namuangruk, Y. Surakhot, N. Kungwan, S. Jungsuttiwong, Microporous Mesoporous Mater. 2017, 241, 372-382.

[696] H. Manzano, L. Gartzia-Rivero, J. Bañuelos, I. López-Arbeloa, J. Phys. Chem. C 2013, 117, 13331-13336.

[697] E. Fois, A. Gamba, G. Tabacchi, ChemPhysChem 2008, 9, 538543

[698] V. Ramamurthy, D. R. Sanderson, D. F. Eaton, J. Am. Chem. Soc. 1993, 115, 10438-10439.

[699] G. Ihlein, F. Schüth, O. Krauß, U. Vietze, F. Laeri, Adv. Mater. 1998 10, 1117-1119.

[700] R. García, V. Martínez-Martínez, R. Sola Llano, I. López-Arbeloa, J. 
Pérez-Pariente, J. Phys. Chem. C 2013, 117, 24063-24070.

[701] R. Sola-Llano, Y. Fujita, L. Gómez-Hortigüela, A. Alfayate, H. Ujii, E. Fron, S. Toyouchi, J. Perez-Pariente, I. Lopez-Arbeloa, V. MartinezMartinez, ACS Photonics 2018, 5, 151-157.

[702] W. Grochala, R. Hoffmann, J. Feng, N. W. Ashcroft, Angew. Chem. Int. Ed. 2007, 46, 3620-3642; Angew. Chem. 2007, 119, 36943717.

[703] L. Merrill, W. A. Bassett, Rev. Sci. Instrum. 1974, 45, 290-294.

[704] R. Miletich, D. R. Allan, W. F. Kuhs, Rev. Mineral. Geochemistry 2000, 41, 445-519.

[705] R. J. Angel, M. Bujak, J. Zhao, G. D. Gatta, S. D. Jacobsen, J. Appl. Crystallogr. 2007, 40, 26-32.

[706] G. D. Gatta, G. Tabacchi, E. Fois, Y. Lee, Phys. Chem. Miner. 2016, 43, 209-216.

[707] P. Lotti, R. Arletti, G. D. Gatta, S. Quartieri, G. Vezzalini, M. Merlini, V. Dmitriev, M. Hanfland, Microporous Mesoporous Mater. 2015, 218, 42-54.

[708] R. M. Hazen, L. W. Finger, J. Appl. Phys. 1984, 56, 1838-1840.

[709] Y. Lee, T. Vogt, J. A. Hriljac, J. B. Parise, J. C. Hanson, S. J. Kim, Nature 2002, 420, 485-489.

[710] Y. Lee, T. Vogt, J. A. Hriljac, J. B. Parise, G. Artioli, J. Am. Chem. Soc. 2002, 124, 5466-5475.

[711] Y. Lee, C. C. Kao, S. J. Kim, H. H. Lee, D. R. Lee, T. J. Shin, J. Y. Choi, Chem. Mater. 2007, 19, 6252-6257.

[712] F. Cailliez, M. Trzpit, M. Soulard, I. Demachy, A. Boutin, J. Patarin A. H. Fuchs, Phys. Chem. Chem. Phys. 2008, 10, 4817

[713] T. Martin, B. Lefevre, D. Brunel, A. Galarneau, F. Di Renzo, F. Fajula, P. F. Gobin, J. F. Quinson, G. Vigier, Chem. Commun. 2002, 24-25.

[714] G. D. Gatta, K. S. Scheidl, T. Pippinger, R. Skála, Y. Lee, R Miletich, Microporous Mesoporous Mater. 2015, 206, 34-41.

[715] C. L. I. M. White, A. R. Ruiz-Salvador, D. W. Lewis, Angew. Chem. Int. Ed. 2004, 43, 469-472; Angew. Chem. 2004, 116, 475-478.

[716] G. D. Gatta, Y. Lee, Phys. Chem. Miner. 2006, 32, 726-732.

[717] D. Seoung, Y. Lee, C.-C. Kao, T. Vogt, Y. Lee, Chem. Mater. 2015 , 27, 3874-3880

[718] P. Lotti, G. D. Gatta, D. Comboni, M. Merlini, L. Pastero, M. Hanfland, Microporous Mesoporous Mater. 2016, 228, 158-167.

[719] G. Fraux, F.-X. Coudert, A. Boutin, A. H. Fuchs, Chem. Soc. Rev. 2017, 46, 7421-7437.

[720] L. Ronchi, A. Ryzhikov, H. Nouali, T. J. Daou, J. Patarin, New J. Chem. 2017, 41, 2586-2592.

[721] N. Desbiens, I. Demachy, A. H. Fuchs, H. Kirsch-Rodeschini, M. Soulard, J. Patarin, Angew. Chem. Int. Ed. 2005, 44, 5310-5313; Angew. Chem. 2005, 117, 5444-5447.

[722] Y. Lee, T. Vogt, Y. Lee, Microporous Mesoporous Mater. 2017, 244 109-118.

[723] A. Y. Likhacheva, Y. V. Seryotkin, A. Y. Manakov, S. V. Goryainov A. I. Ancharov, M. A. Sheromov, Am. Mineral. 2007, 92.

[724] D. Comboni, G. D. Gatta, P. Lotti, M. Merlini, M. Hanfland, Microporous Mesoporous Mater. 2018, 263, 86-95.

[725] Y. Kim, J. Choi, T. Vogt, Y. Lee, Am. Mineral. 2018, 103, 175-178.

[726] Y. Lee, S. J. Kim, D. C. Ahn, N. S. Shin, Chem. Mater. 2007, 19 2277-2282.

[727] Y. Lee, Y. Lee, D. Seoung, Am. Mineral. 2010, 95, 1636-1641.

[728] Y. Lee, Y. Lee, D. Seoung, J. H. Im, H. J. Hwang, T. H. Kim, D. Liu, Z. Liu, S. Y. Lee, C. C. Kao, T. Vogt, Angew. Chem. Int. Ed. 2012, 51, 4848-4851; Angew. Chem. 2012, 124, 4932-4935.

[729] J. N. Grima, R. Gatt, V. Zammit, J. J. Williams, K. E. Evans, A Alderson, R. I. Walton, J. Appl. Phys. 2007, 101, 86102.
[730] A. Kremleva, T. Vogt, N. Rösch, J. Phys. Chem. C 2013, 117 19020-19030.

[731] A. Kremleva, T. Vogt, N. Rösch, J. Phys. Chem. C 2014, 118 22030-22039.

[732] V. Schettino, R. Bini, Chem. Soc. Rev. 2007, 36, 869-880.

[733] M. Citroni, M. Ceppatelli, R. Bini, V. Schettino, High Press. Res. 2002, 22, 507-510

[734] P. Enzel, J. J. Zoller, T. Bein, A. G. MacDiarmid, A. J. Heeger, J. Chem. Soc. Chem. Commun. 1992, 0, 633-635.

[735] M. Santoro, F. A. Gorelli, R. Bini, J. Haines, A. van der Lee, Nat Commun. 2013, 4, 1557.

[736] M. Santoro, K. Dziubek, D. Scelta, M. Ceppatelli, F. A. Gorelli, R. Bini, J. M. Thibaud, F. Di Renzo, O. Cambon, J. Rouquette, P. Hermet, A. Van Der Lee, J. Haines, Chem. Mater. 2015, 27, 64866489 .

[737] M. Santoro, D. Scelta, K. Dziubek, M. Ceppatelli, F. A. Gorelli, R. Bini, G. Garbarino, J. M. Thibaud, F. Di Renzo, O. Cambon, P. Hermet, J. Rouquette, A. Van Der Lee, J. Haines, Chem. Mater. 2016, 28, 4065-4071.

[738] J. Im, Y. Lee, D. A. Blom, T. Vogt, Y. Lee, Dalt. Trans. 2016, 45 $1622-1630$.

[739] P. Lotti, G. D. Gatta, M. Merlini, H.-P. Liermann, Zeitschrift für Krist. - Cryst. Mater. 2015, 230, 201-211.

[740] R. Arletti, L. Leardini, G. Vezzalini, S. Quartieri, L. Gigli, M. Santoro, J. Haines, J. Rouquette, L. Konczewicz, Phys. Chem. Chem. Phys. 2015, 17, 24262-24274.

[741] D. Santamaría-Pérez, T. Marqueño, S. MacLeod, J. Ruiz-Fuertes, D. Daisenberger, R. Chuliá-Jordan, D. Errandonea, J. L. Jordá, F. Rey, C. McGuire, A. Mahkluf, A. Kavner, C. Popescu, Chem. Mater. 2017, 29, 4502-4510.

[742] J. Richard, S. L. Cid, J. Rouquette, A. Van Der Lee, S. Bernard, J. Haines, J. Phys. Chem. C 2016, 120, 9334-9340.

[743] A. Corma, F. Rey, J. Rius, M. J. Sabater, S. Valencia, Nature 2004, 431, 287-290.

[744] R. Arletti, L. Ronchi, S. Quartieri, G. Vezzalini, A. Ryzhikov, H. Nouali, T. J. Daou, J. Patarin, Microporous Mesoporous Mater. 2016, 235, 253-260.

[745] P. A. Vaughan, Acta Crystallogr. 1966, 21, 983-990.

[746] A. Alberti, C. Sabelli, Zeitschrift für Krist. 1987, 178, 249-256.

[747] R. Arletti, G. Vezzalini, S. Quartieri, F. Di Renzo, V. Dmitriev, Microporous Mesoporous Mater. 2014, 191, 27-37.

[748] C. Wang, P. Bai, J. I. Siepmann, A. E. Clark, 2014

[749] P. Bai, M. Y. Jeon, L. Ren, C. Knight, M. W. Deem, M. Tsapatsis, J. I. Siepmann, Nat. Commun. 2015, 6, 5912.

[750] R. F. DeJaco, P. Bai, M. Tsapatsis, J. I. Siepmann, Langmuir 2016 32, 2093-2101.

[751] N. Mittal, P. Bai, J. I. Siepmann, P. Daoutidis, M. Tsapatsis, J. Memb. Sci. 2017, 540, 464-476.

[752] R. Arletti, E. Fois, G. Tabacchi, S. Quartieri, G. Vezzalini, Adv. Sci. Lett. 2017, 23, 5966-5969.

[753] C. H. Wang, P. Bai, J. I. Siepmann, A. E. Clark, J. Phys. Chem. C 2014, 118, 19723-19732.

[754] I. E. Collings, E. Bykova, M. Bykov, S. Petitgirard, M. Hanfland, D Paliwoda, L. Dubrovinsky, N. Dubrovinskaia, ChemPhysChem 2016, 17, 3369-3372.

[755] S. C. McKellar, S. A. Moggach, IUCr, Acta Crystallogr. Sect. B Struct. Sci. Cryst. Eng. Mater. 2015, 71, 587-607.

[756] E. A. Kapustin, S. Lee, A. S. Alshammari, O. M. Yaghi, ACS Cent Sci. 2017, 3, 662-667.

[757] T. D. Bennett, P. Simoncic, S. A. Moggach, F. Gozzo, P. Macchi, D. 
A. Keen, J.-C. Tan, A. K. Cheetham, Chem. Commun. 2011, 47, 7983-7985.

[758] W. Li, M. R. Probert, M. Kosa, T. D. Bennett, A. Thirumurugan, R. P. Burwood, M. Parrinello, J. A. K. Howard, A. K. Cheetham, J. Am. Chem. Soc. 2012, 134, 11940-11943.

[759] S. Sobczak, A. Katrusiak, Cryst. Growth Des. 2018, 18, 1082-1089.

[760] J. Im, D. Seoung, G. C. Hwang, J. W. Jun, S. H. Jhung, C.-C. Kao T. Vogt, Y. Lee, Chem. Mater. 2016, 28, 5336-5341.

[761] A. U. Ortiz, A. Boutin, A. H. Fuchs, F.-X. Coudert, J. Chem. Phys. 2013, 138, 174703.

[762] J. Sotelo, C. H. Woodall, D. R. Allan, E. Gregoryanz, R. T. Howie, K. V. Kamenev, M. R. Probert, P. A. Wright, S. A. Moggach, Angew. Chem. Int. Ed. 2015, 54, 13332-13336; Angew. Chem. 2015, 127 13530-13534.

[763] C. L. Hobday, R. J. Marshall, C. F. Murphie, J. Sotelo, T. Richards, D. R. Allan, T. Düren, F. X. Coudert, R. S. Forgan, C. A. Morrison, S. A. Moggach, T. D. Bennett, Angew. Chem. Int. Ed. 2016, 55, 24012405; Angew. Chem. 2016, 128, 2447-2451.

[764] J. S. Lee, B. Vlaisavljevich, D. K. Britt, C. M. Brown, M. Haranczyk J. B. Neaton, B. Smit, J. R. Long, W. L. Queen, Adv. Mater. 2015, 27, 5785-5796.

[765] M. R. Ryder, B. Civalleri, J.-C. Tan, Phys. Chem. Chem. Phys. 2016 18, 9079-9087.

[766] W. F. Perger, J. Criswell, B. Civalleri, R. Dovesi, Comput. Phys. Commun. 2009, 180, 1753-1759.

[767] A. Erba, D. Caglioti, C. M. Zicovich-Wilson, R. Dovesi, J. Comput. Chem. 2017, 38, 257-264.

[768] J.-C. Tan, B. Civalleri, A. Erba, E. Albanese, CrystEngComm 2015, 17, 375-382.

[769] L. Öhrström, ACS Cent. Sci. 2017, 3, 528-530.

[770] J. L. Jordá, F. Rey, G. Sastre, S. Valencia, M. Palomino, A. Corma, A. Segura, D. Errandonea, R. Lacomba, F. J. Manjón, Ó. Gomis, A. K. Kleppe, A. P. Jephcoat, M. Amboage, J. A. RodríguezVelamazán, Angew. Chem. Int. Ed. 2013, 52, 10458-10462; Angew. Chem. 2013, 125, 10652-10656.

[771] T. H. Noh, O. S. Jung, Acc. Chem. Res. 2016, 49, 1835-1843.

[772] L. Bertrand, C. Gervais, A. Masic, L. Robbiola, Angew. Chem. Int. Ed. 2018, DOI 10.1002/anie.201709303; Angew. Chem. 2018, DOI 10.1002/ange.201709303.

[773] J. Bujdák, Clay Miner. 2015, 50, 549-571.

[774] M. Ogawa, R. Takee, Y. Okabe, Y. Seki, Dye. Pigment. 2017, 139 , 561-565.
[775] M. Matejdes, D. Himeno, Y. Suzuki, J. Kawamata, Appl. Clay Sci. 2017, 140, 119-123.

[776] F. Olivero, F. Carniato, C. Bisio, L. Marchese, Chem. Asian J. 2014 9, 158-165.

[777] U. Costantino, F. Costantino, F. Elisei, L. Latterini, M. Nocchetti, Phys. Chem. Chem. Phys. 2013, 15, 13254-13269.

[778] R. A. Schoonheydt, Appl. Clay Sci. 2016, 131, 107-112.

[779] E. Duque-Redondo, H. Manzano, N. Epelde-Elezcano, V. MartínezMartínez, I. López-Arbeloa, Chem. Mater. 2014, 26, 4338-4345.

[780] N. Epelde-Elezcano, E. Duque-Redondo, V. Martínez-Martínez, H. Manzano, I. López-Arbeloa, Langmuir 2014, 30, 10112-10117.

[781] N. Epelde-Elezcano, V. Martínez-Martínez, E. Duque-Redondo, I. Temiño, H. Manzano, I. López-Arbeloa, Phys. Chem. Chem. Phys. 2016, 18, 8730-8738.

[782] P. Woodtli, S. Giger, P. Müller, L. Sägesser, N. Zucchetto, M. J. Reber, A. Ecker, D. Brühwiler, Dye. Pigment. 2018, 149, 456-461.

[783] X. Guo, P. Yin, H. Yang, Microporous Mesoporous Mater. 2018, 259 123-133.

[784] S. Sansuk, S. Srijaranai, S. Srijaranai, Environ. Sci. Technol. 2016, 50, 6477-6484.

[785] M. E. McBriarty, J. A. Soltis, S. Kerisit, O. Qafoku, M. E. Bowden, E. J. Bylaska, J. J. De Yoreo, E. S. Ilton, Environ. Sci. Technol. 2017, 51, 4970-4977.

[786] X. Y. Li, Y. Jiang, X. Q. Liu, L. Y. Shi, D. Y. Zhang, L. B. Sun, ACS Sustain. Chem. Eng. 2017, 5, 6124-6130.

[787] B. Dong, R. Retoux, V. de Waele, S. G. Chiodo, T. Mineva, J. Cardin, S. Mintova, Microporous Mesopor. Mater. 2017, 244, 74-82.

[788] S. e. Gul, D. Cody, A. Kharchenko, S. Martin, S. Mintova, J. Cassidy, I. Naydenova, Microporous Mesoporous Mater. 2018, 261, 268-274.

[789] A. Z. Ruiz, D. Brühwiler, T. Ban, G. Calzaferri, Monatshefte für Chemie 2005, 136, 77-89.

[790] X. Chen, X. Bu, Q. Lin, C. Mao, Q. G. Zhai, Y. Wang, P. Feng, Chem. Eur. J. 2017, 23, 11913-11919.

[791] G. Tabacchi, E. Fois, D. Barreca, A. Gasparotto, Int. J. Quantum Chem. 2014, 114, 1-7.

[792] Q. Cui, R. Hernandez, S. E. Mason, T. Frauenheim, J. A. Pedersen, F. Geiger, J. Phys. Chem. B 2016, 120, 7297-7306.

[793] G. M. Whitesides, Angew. Chem. Int. Ed. 2018, DOI 10.1002/anie.201800684; Angew. Chem. 2018, DOI 10.1002/ange.201800684. 


\section{REVIEW}

Under tight confinement in nanosized cavities, molecules may self-assemble creating beautiful patterns and technologically useful materials. The complexity and the potential of nanospace-confined supramolecular organization is illustrated by natural and artificial examples, highlighting, beside diversity, their common molecular bases and interconnections.

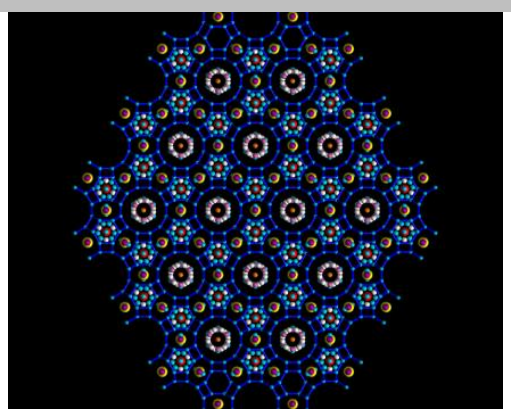

Gloria Tabacchi*

Supramolecular organization in confined nanospaces 